\title{
BAROMETRIC PUMPING OF BURIAL TRENCH SOIL GASES INTO THE ATMOSPHERE AT THE 740-G SANITARY LANDFILL (U)
}

by

D. E. Wyatt, R. J. Pirkle, and D. J. Masdea

Westinghouse Savannah River Company

Savannah River Site

Aiken, South Carolina 29808

This report was prepared in connection with work done under Contract No. DE-AC09-89SR18035 with the U.S. Department of Energy. By acceptance of this report, the publisher and/or recipient acknowledges the U.S. Government's right to retain a nonexclusive, royalty-free license in and to any copyright covering this report, along with the right to reproduce and to authorize others to reproduce all or part of the copyrighted report. 


\section{DISCLAIMER}

This report was prepared by Westinghouse Savannah River Company (WSRC) for the United States Department of Energy under Contract No. DE-AC09-89SR18035 and is an account of work performed under that contract. Neither the United States Department of Energy, nor WSRC, nor any of their employees makes any warranty, express or implied, or assumes any legal liability or responsibility for the accuracy, completeness, or usefuiness, of any information, apparatus, or product or process disclosed herein or represents that its use will not intringe privately owned rights. Reference herein to any specific commercial product, process, or service by trademark, name, manufacturer or otherwise does not necessarily constitute or imply endorsement, recommendation, or favoring of same by WSRC or by the United States Government or any agency therefof. The views and opinions of the authors expressed herein do not necessarily state or reflect those of the United States Government or any agency thereof.

Printed in the United States of America

Available from

National Technical Information Service

U. S. Department of Commerce 5285 Port Royal Road Springfield, VA 22161 
WSRC-RP-92-877, Rev I

\title{
Barometric Pumping of Burial Trench Soil Gases Into the Atmosphere at the 740-G Sanitary Landfill (U)
}

\author{
D. E. Wyatt \\ R. J. Pirkle \\ D. J. Masdea
}

Westinghouse Savannah River Company

Savannah River Site

Aiken, SC 29808 
This document was modified to a Revision 1 status to meet DOE release requirements. 


\section{IABLE OF CONTENTS}

List of Figures

List of Tables

Executive Summary

I. Introduction

II. Background

III. Experimental

A. Sampling Methods $\quad 8$

B. Analytical Methods 9

C. Accuracy 9

D. Precision $\quad \cdots$

E. Minimum Detection Levels 9

F. Blanks $\quad 10$

G. Data on Magnetic Disk 10

$\begin{array}{ll}\text { V. Results } & 10\end{array}$

VI. Conclusions and Recommendations 13

VII. Figures and Tables

VIII. Appendix I

Sampling Method SMI

Analytical Method AMI 


\section{TABLE OF CONTENTS (cont.)}

IX. Appendix II

Field Sample Logs

\section{LIST OF FIGURES}

Figure 1. Locations of Groundsheets for the 740-G Landfill Groundsheet Study

Figure 2. Methane Concentrations (ppmv) Under Each Groundsheet as a Function of Time

Figure 3. Soil Gas Percent Methane at the 740-G Landfill

Figure 4. Representative Chromatogram of $\mathrm{Cl}_{1}-\mathrm{C}_{4}$ Hydrocarbons Under Groundsheet \#1.

Figure 5. Representative Chromatogram of $\mathrm{Cl}_{1}-\mathrm{C}_{4}$ Hydrocarbons Under Groundsheet \#2.

Figure 6. Representative Chromatogram of $\mathrm{Cl}_{\mathrm{F}} \mathrm{C}_{4}$ Hydrocarbons Under Groundsheet \#3.

Figure 7. Representative Chromatogram of $\mathrm{Cl}_{1}-\mathrm{C}_{4} \mathrm{Hydrocarbons}$ Under Groundsheet \#4.

Figure 8. Representative Gas Chromatogram of $\mathrm{Cl}_{\mathrm{l}}-\mathrm{C}_{4}$ Hydrocarbon Standard " $\mathrm{M}$ " and Standard "224".

Figure 9. Chromatogram of $\mathrm{C}_{1}-\mathrm{C}_{4}$ Hydrocarbon Standard " $\mathrm{M}$ " Diluted 200:1 


\section{LIST OF FIGURES (continued)}

Figure 10. Representative Chromatogram of System Blank \#5

Figure 11. Representative Chromatogram of System Blank \#19

Figure 12. Methane Under Groundsheet \#2 vs Barometric Pressure

Figure 13. Ethane Under Groundsheet \#2 vs Barometric Pressure

Figure 14. Ethylene Under Groundsheet \#2 vs Barometric Pressure

Figure 15. Propane Under Groundsheet \#2 vs Barometric Pressure

Figure 16. Propylene Under Groundsheet \#2 vs Barometric Pressure

Figure 17. i-Butane Under Groundsheet \#2 vs Barometric Pressure

Figure 18. Methane Under Groundsheet \#I vs Barometric Pressure

Figure 19. Ethane Under Groundsheet \#l vs Barometric Pressure

Figure 20. Propane Under Groundsheet \#l vs Barometric Pressure

Figure 21. Methane Under Groundsheet \#3 vs Barometric Pressure

Figure 22. Propane Under Groundsheet \#3 vs Barometric Pressure

Figure 23. Methane Under Groundsheet \#4 vs Barometric Pressure

Figure 24. Propane Under Groundsheet \#4 vs Barometric Pressure 
LIST OF FIGURES (continued)

Figure 25. VOC's Under Groundsheet \#2 During a Period of Falling Barometric Pressure.

Figure 26. VOC's in the Burial Trench Beneath Groundsheet \#2 from Soil Gas Site Location \#52

\section{LIST OF TABLES}

Table 1. $\quad \mathrm{C}_{1}-\mathrm{C}_{4}$ Hydrocarbon Concentrations (ppmv) and System Blanks in the 740-G Landfill Groundsheet Study

Table 2. Analyses of $\mathrm{C}_{\mid}-\mathrm{C}_{4}$ Hydrocarbon Standard " $\mathrm{M}$ " and Standard "224" During the 740-G Landfill Groundsheet Study

Table 3. Analyses of System Blanks

Table 4. Light Hydrocarbon Concentrations in Soil Gas Survey Sites Near Each Groundsheet 


\section{EXECUTIVE SUMMARY}

In 1991, a soil gas survey was performed at the Savannah River Site Sanitary Landfill as part of the characterization efforts required under the integrated Resource Conservation and Recovery Act (RCRA) Facility Investigation and Comprehensive Environmental Resource Conservation and Recovery Act (CERCLA) Remedial Investigation (RFI/RI) program. This survey identified several areas of the landfill that were releasing volatile organic compounds to the atmosphere at levels exceeding regulatory standards. Knowledge of the rates of VOC outgassing is necessary to protect site workers, provide input into the human health and environmental risk assessment documents and provide input into the remedial design scenario. Data from the characterization soil gas survey is presented in WSRC-RP-92-878.

It is suspected that the outgassing of volatile organics varies with barometric pressure, therefore an experiment was designed to evaluate selected areas of the landfill over time and compare the results with the measured barometric pressure at the time of the sampling. To accomplish this, air under four groundsheets was sampled and analyzed for the light hydrocarbons, $\mathrm{C}_{1} \mathrm{C}_{4}$. Selected samples were analyzed for a number of volatile organic species.

The results of this study confirm that during periods of decreasing barometric pressure, VOC gaseous components of the burial trenches migrate through the soil cover into the near surface atmosphere. This variable migration may affect remedial designs such as clay barrier caps and methane ventilation systems. It is suggested that these data be further studied to determine the actual amounts of these volatile species which are injected into the atmosphere by barometric pumping at this and other "burial grounds" both at SRS and in the public sector as well. 


\section{INTRODUCTION}

Data from the completion of a soil gas survey of the 740-G Sanitary Landfill (data provided in WSRC-RP-92-878), in support of the RCRACERCLA Facility Investigation/Remedial Investigation program, found several areas where the VOC emissions from the landfill trenches exceeded regulatory levels. Because these emissions could affect the human health risk assessment scenarios and also possibly affect remedial design criteria, an experiment was designed to evaluate the type and timing of soil gas emissions by barometric pumping. Four groundsheets were installed at selected locations at the landfill to test the hypothesis that landfill gases might be escaping through the soil cap over the burial trenches and that such gaseous migration was related to changes in barometric pressure. Groundsheets were utilized to restrict dilution and mixing of the gasses with air. Locations of each of the four groundsheets at the landfill relative to site locations of the landfill soil gas survey are shown on Figure 1. Samples were taken from beneath each groundsheet every four hours over a 2 week period between January 5 - 19, 1991.

These samples were analyzed for the light hydrocarbons including methane, ethane, propane, i-butane, n-butane, ethylene, and propylene. Methane was the component in highest concentration found in the burial trenches during the landffill soil gas survey. In some areas, burial trench methane exceeded $60 \%$ of the total gas. Light hydrocarbon concentrations (ppmv) under each of the four groundsheets are presented in Table 1 and methane concentrations are shown as a function of time on Figure 2.

\section{BACKGROUND}

A soil gas survey conducted in November and December, 1990 of some 252 locations at the 740-G Landfill, as shown on Figure 1, revealed the presence of numerous volatile organic species in the soil air of the burial trenches, e.g. see Figure 26. Not surprisingly, methane gas, presumably resulting from the bacterial degradation of cellulose and other organic materials, was found in 
large concentrations, exceeding $60 \%$ of the total soil air in some areas of the landfill as shown on the contour map of percent methane on Figure 3. Details of this soil gas survey of the 740-G Landfill are discussed in a separate report (WSRC-MS-92-878).

The observations of the large concentrations of volatile species, particularly methane, in the landfill burial trenches, suggested to SRS personnel the potential that these gases could move through the $2.5-3.0$ foot soil layer covering the burial trenches, particularly during periods of rapidly decreasing barometric pressure. This suggestion was based on experience with soil gas observations and measurements of similar soil air/atmospheric pressure equilibrations near a leaking hydrocarbon storage well .

While it is a rather elementary concept that reduction of atmospheric pressure at the earths surface would cause air from the pore volume of the soil to rapidly equilibrate, resulting in flow of soil air into the atmosphere, it is a phenomenon which, to our knowledge, has not been widely studied. It is hoped that this dataset will provide impetus for further evaluation and quantification of the effects of barometric pumping and its implications with respect to the "storage" of volatile species in near surface soils.

\section{EXPERIMENTAL}

In order to implement this study, four $8^{\prime} \times 8^{\prime}$ groundsheets were constructed. Each groundsheet consisted of an $8^{\prime} \times 8^{\prime}$ frame made of $2^{\prime \prime} \times 4^{\prime \prime} \times 8^{\prime}$ lumber which was covered with two sheets of $4^{\prime} \times 8^{\prime} \times 3 / 4^{\prime \prime}$ strand board. These materials were nailed together in a manner calculated to prevent or significantly impede convective flow of gases under the groundsheets by the wind. No attempt was made to make the groundsheets airtight, i.e., no glue or other sealants were used. It is acknowledged that some convection may have occurred, particularly toward the end of the sampling period due to the effects of weather on the groundsheets. 
When each groundsheet was installed at the landfill, a trench, 1" to 1.5" deep, was dug to the dimensions of the frame. After the groundsheet was in place in the trench, the soil removed from the trench was replaced around the outside of the groundsheet to impede convective flows of air under the edge.

A $1 / 4$ " o.d. nylon sampling tube attached to the underside (center) of each groundsheet, was brought out under one edge and secured to the top of the groundsheet to allow ease of sampling. This tube was capped so as to prevent water from entering the tube during periods of rainfall.

Sampling from under the groundsheets began at approximately 10 am on January 5, 1991 and was repeated at a nominal interval of 4 hours until January 19, 1991. A total of 369 samples (including duplicates) were taken. A few samples from under groundsheet \#4 could not be taken on January 15-16 when it was submerged by a heavy rain. This water was drained on January 16 and sampling was resumed.

Barometric pressure as a function of time was supplied by SRS from a meteorological station on the site. In hindsight it would have been useful to have recorded rainfall amounts at the landfill. Certainly, the water content of the soil affects its permeability and thus its response to barometric pressure changes.

\section{QUALITY ASSURANCE}

The quality assurance/quality control procedures of the sampling subcontractor were followed for all field and laboratory analytical work. Documentation of applicable sampling and analytical methods and associated quality assurance procedures are included in this report in Appendix I. 


\section{A. Sampling Methods}

Methods and quality assurance procedures used to obtain groundsheet air samples are a modification of those used for soil gas samples in Sampling Method SMI in Appendix I. In this case, since no hole was required, the sampling syringe was simply removed from the sample probe described in SM1 and was attached to the $1 / 4$ " nylon sampling tube as each groundsheet was sampled.

Samples were collected in previously evacuated $22 \mathrm{ml}$ bottles fitted with butyl rubber septa. A duplicate sample and a blank was collected for every 10 samples. Field sample logs which document the details of the sample collection are in Appendix II.

\section{B. Analytical Methods}

Methods and quality assurance procedures for the analysis of light hydrocarbon soil gas samples are documented in Analytical Method AMI. Representative chromatograms of $\mathrm{C}_{\mathrm{l}}-\mathrm{C}_{4}$ hydrocarbon samples from this data set are shown on Figures 4-7.

\section{Accuracy}

Calibration of the $\mathrm{C}_{1}-\mathrm{C}_{4}$ hydrocarbon gas chromatograph was accomplished using a certified commercial standard (Matheson Gas Products Inc.) which is of the order $10 \mathrm{ppm}$ for methane and $1 \mathrm{ppm}$ for the other light hydrocarbons and is called Standard "M"; and a second commercial standard (Scotty Specialty Gases, Can Mix 224, ICN \#35) which contains only the $\mathrm{Cl}_{\mathrm{I}}-\mathrm{C}_{4}$ straight chain hydrocarbons and is of the order $1000 \mathrm{ppmv}$ for each hydrocarbon. A chromatogram of these standards (Standard " $\mathrm{M}$ " and "224") is shown on Figure 8. 


\section{Precision}

We have estimated the working precision of this data from the repetitive analyses of hydrocarbon standards" $M$ " and "224" as shown on Table 2. The percent standard deviation of the mean of 39 analyses of Standard " $M$ " is less than $5 \%$ for each of the seven compounds. The percent standard deviation of the mean of 4 analyses of Standard "224" was $\leq 2 \%$.

\section{E. Minimum Detection Levels}

Minimum detection levels reported in this survey for the light hydrocarbons, $\mathrm{C}_{\mathrm{F}}$ $\mathrm{C}_{4}$, are $5 \mathrm{ppb}$. A $5 \mathrm{ppb}$ standard (Microseeps Standard M diluted 200:1) is reliably determined with $S / N>2$. A chromatogram of the analysis of this diluted standard is shown on Figure 9.

\section{F. Blanks}

Results of the analyses of system blanks are included on Table 3 and representative chromatograms are shown on Figures 10 and 11.

\section{G. Data On Magnetic Disk}

All analytical data, including samples, duplicates, standards, and blanks are provided (one copy) on magnetic disk. All data are in units of parts per million by volume.

All data are in Lotus 123 (Release 2, 1985) spreadsheet format using DOS (Version 4.01). All analytical data were accumulated and processed automatically via a gas chromatographic data system. The processed data is transferred directly into the Lotus format thus ensuring the quality of the data. 


\section{RESULTS}

The results of the analyses of light hydrocarbons in the air under each of the four groundsheets at the 740-G Landfill is presented in Table 1 and the methane concentration data is presented on Figure 2. Concentrations of the $\mathrm{Cl}_{\mathrm{F}}$ $\mathrm{C}_{4}$ hydrocarbons which were determined in the landfill soil gas survey sites nearest each of the four groundsheets are shown in Table 4.

Upon inspection of Figure 2, it is clear that methane concentrations under all groundsheets varied considerably over the two weeks of observations; and that the measured concentrations under groundsheet \#2 are considerably larger than those measured under groundsheets 1,3 , and 4 . The methane concentrations under groundsheets 1,3 , and 4 are similar in magnitude. More careful study of Figure 2, reveals that changes in methane concentrations under all groundsheets are in phase, despite the obvious differences in magnitude mentioned above.

We have already suggested that one possible reason for observation of soil gas hydrocarbons in the air under the groundsheets was pressure equilibration of soil air with the atmosphere during periods of rapidly decreasing barometric pressure. Presented in Figures 12 - 24 are concentrations of selected components of the light hydrocarbons (ppmv) under each of the groundsheets and barometric pressure in millibars as a function of time.

Methane under groundsheet \#2 and barometric pressure as a function of time is shown on Figure 12. It is immediately obvious that methane under this groundsheet increases dramatically during periods of decreasing barometric pressure. Closer study of Figure 12 reveals that even short periods of stable pressure in an overall decreasing pressure regime, causes cessation of the methane increase under the groundsheet. For example, the overall increase in methane between 10 am on January 6 and 6 am on January 7 , is punctuated by a significant decreases in methane, which occurs during a short period of stable pressure between $6 \mathrm{pm}$ on January 6 and 2 am on January 7. Again the 
increase in methane which begins late on January 10 , peaks and decreases mid-day on January 11 before increasing dramatically early on January 12 . (Note that on the time axis, each tick mark interval is four hours beginning at 10 am January 5. The tick mark opposite the indicated date January $6-19$ is 2 am on that date.) The increases in methane after January 15 seem somewhat muted compared to the earlier data when the large pressure drop between January 15 and 16 is considered. This may be due to increased loss of methane from the groundsheet as its physical integrity degraded due to weathering, or to the effects of increased soil moisture resulting from the heavy rains during this period. Increases in soil moisture no doubt decrease the permeability of the soil and impede the pressure equilibration which causes the flow of soil air through the soil/atmosphere boundary. The heavier hydrocarbon homologs; ethane, ethylene, propane, propylene, and i-butane; also migrate through the soil/atmosphere boundary at groundsheet \#2 as shown in Figures $13-17$.

The effects of barometric pumping for selected hydrocarbons at groundsheets 1 , 3 , and 4 are shown in Figures $18-24$. Levels of hydrocarbons measured at these groundsheets are smaller than those observed at groundsheet \#2. This is despite the fact that the measured soil gas hydrocarbons in the burial trench beneath groundsheet $\# 1$ are higher than in the burial trench beneath groundsheet \#2 as shown on Table 4 and Figure 3. This points to the important role of permeability in the overall process of vertical migration of gases in near surface soils.

Although not a part of the formal scope of this study, we were interested to qualitatively determine if the VOC's in the landfill burial trenches also migrated through the soil cap into the atmosphere. Selected sample bottles from groundsheet \#2 from which we had already analyzed a portion of the contents for the light hydrocarbons were recovered. A quick check revealed that those selected still retained a slight positive pressure suggesting that little if any contamination of their contents had occurred since the $\mathrm{C}_{1}-\mathrm{C}_{4}$ analysis in early January. Pressure in each of these $22 \mathrm{ml}$ bottles was bled to atmospheric 
pressure using a syringe needle and $22 \mathrm{ml}$ of nitrogen gas was then added with the sample syringe. This effectively diluted the concentration of the components of the sample by a factor of 2 relative to its original concentration before analysis of the light hydrocarbons. The selected samples were those collected across the large methane excursions which occurred between midnight on January 10 and $6 \mathrm{pm}$ on January 12. Shown in Figure 25 are flame ionization detector profiles with three successively expanded scales of the sample collected under groundsheet \#2 at $2 \mathrm{pm}$ on January 11 during a methane concentration peak. The flame ionization detector profile of the VOC's from the burial trench beneath groundsheet \#2 is shown in Figure 26. Although the match is not one-to-one, these data clearly show that volatile species which migrate through the soil/atmosphere boundary during periods of rapidly falling barometric pressure, are not limited to the light hydrocarbons, "but extend to many if not all components of the burial trench gases. From the uppermost trace of the chromatogram of groundsheet gases in Figure 25, it is obvious that methane is by far the dominant organic species present. It may be that the higher molecular weight components are assisted in their migration through the soil cap by the large excess of methane.

These data could be modeled to set some limits on the total amounts of volatile species released at the 740-G Landfill per year and to assess the significance of these releases on the environment. The results of such a study and its implications for other "burial grounds" both at SRS and nationwide would seem to be of more than passing environmental interest. 


\section{CONCLUSIONS AND RECOMMENDATION}

Volatile species including, but not limited to the light hydrocarbons, were found to migrate through the soil/atmosphere boundary at the 740-G Landfill during periods of rapidly decreasing barometric pressure. Short periods of stable pressure during overall declining pressure regimes were sufficient to temporarily halt the vertical migration. The data suggest the importance of soil permeability to the overall rate of vertical migration.

It is suggested that these observations conclusively demonstrate that barometric pumping is a fundamental process which plays an important role in the movement of volatile species in the near surface soil and should be considered when evaluating VOC data for regulatory risk assessments and remedial/closure design. It is recommended that these data be studied and perhaps extended to determine the implications of this process to the injection of volatile species into the atmosphere. 
Figures and Tables 


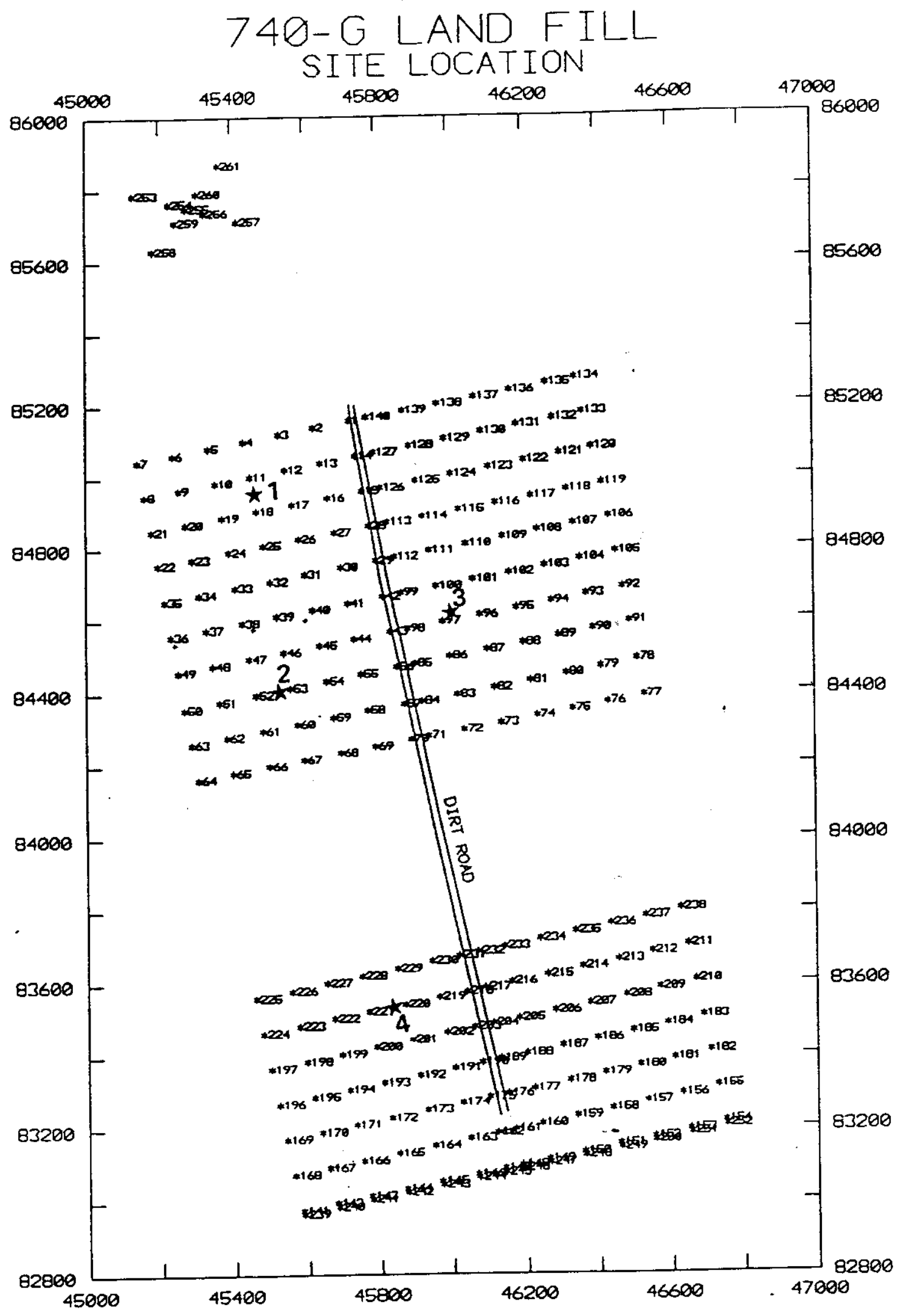

Figure 1. Locations $(\star)$ of Groundsheets for the 740-G Landfill. Groundsheet study Relative to site Locations for the soil Gas Survey 


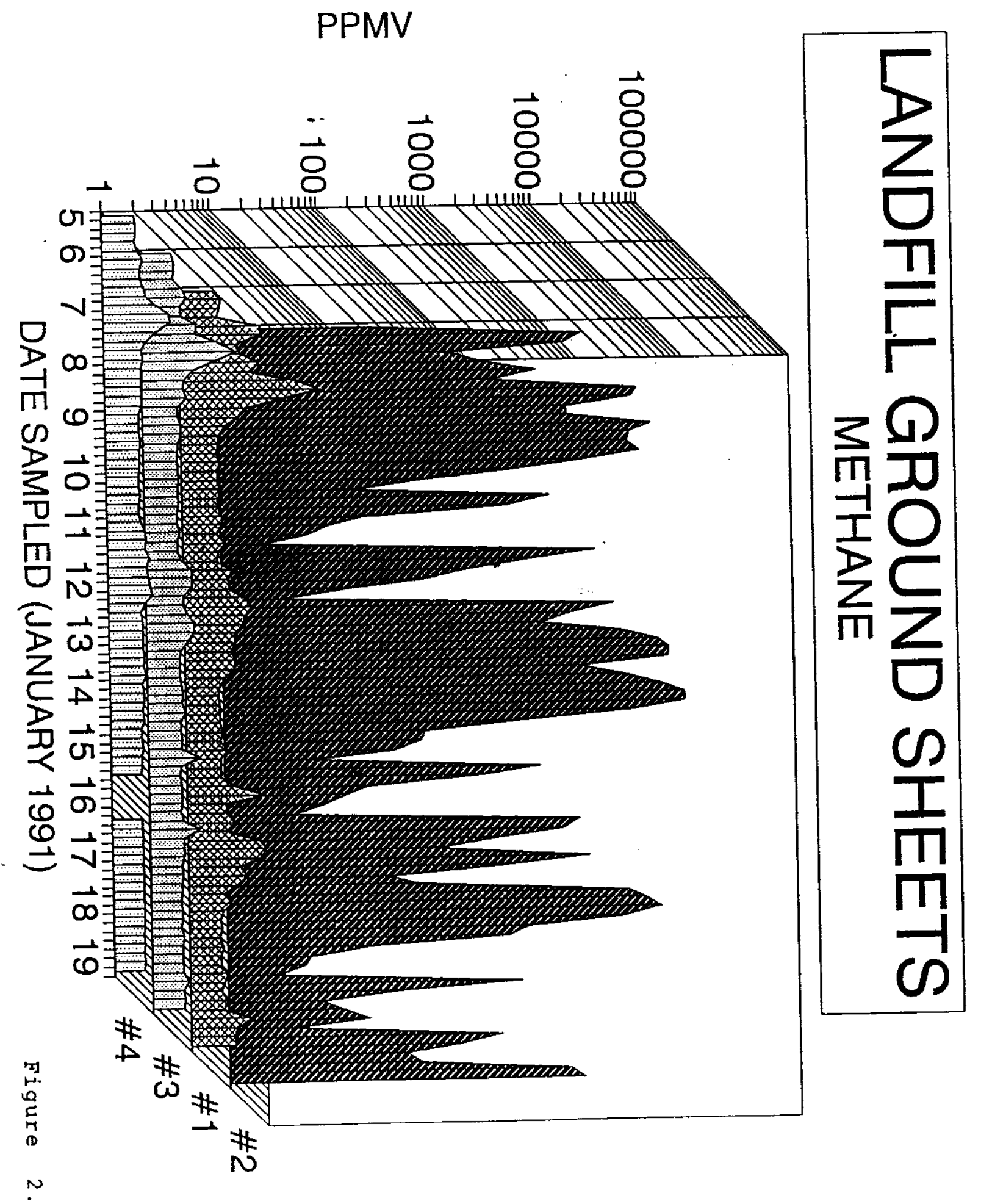


740-G LANDFILL ( NORTH AREA)

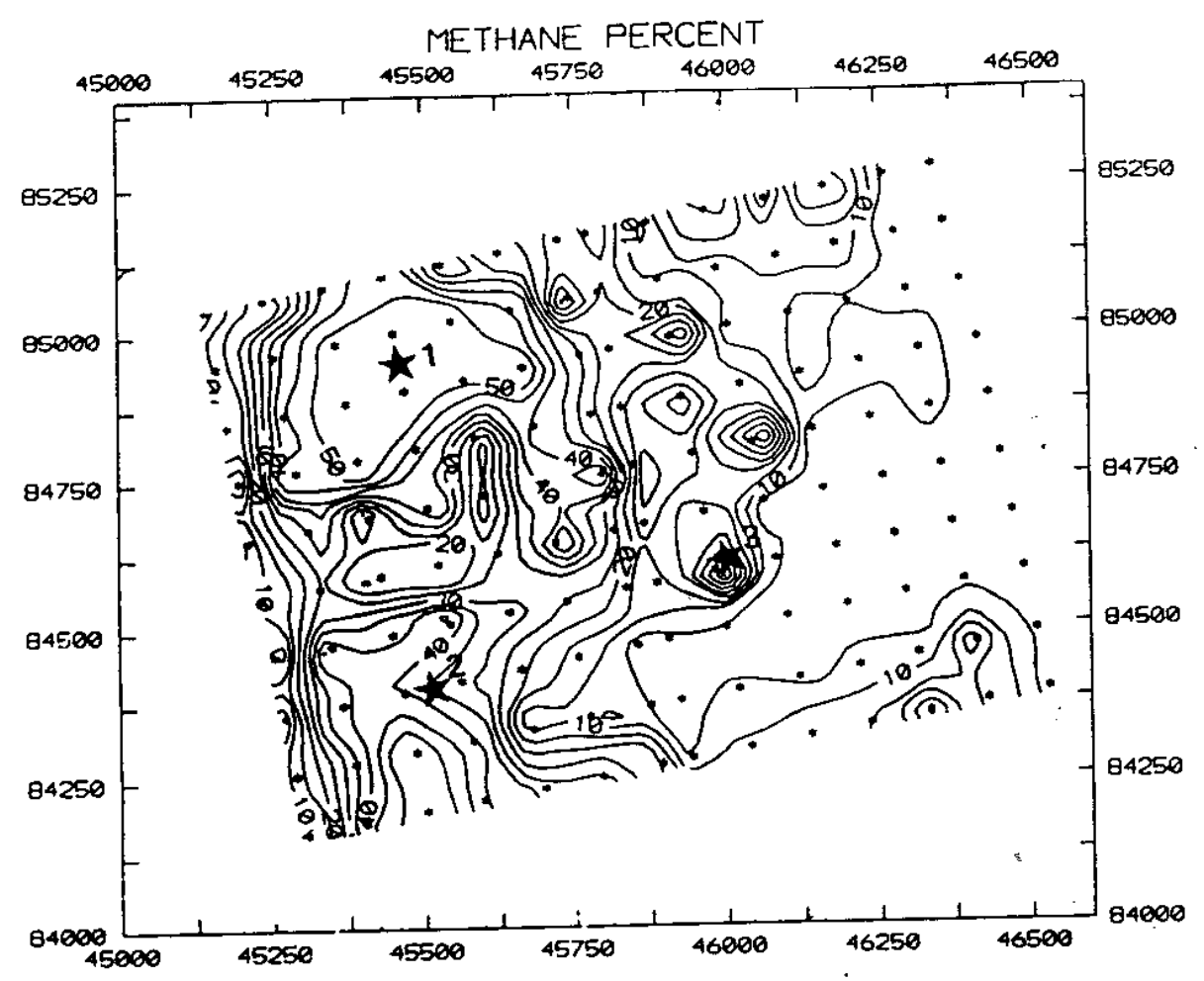

\section{0-G LANDFILL (SOUTH AREA)}

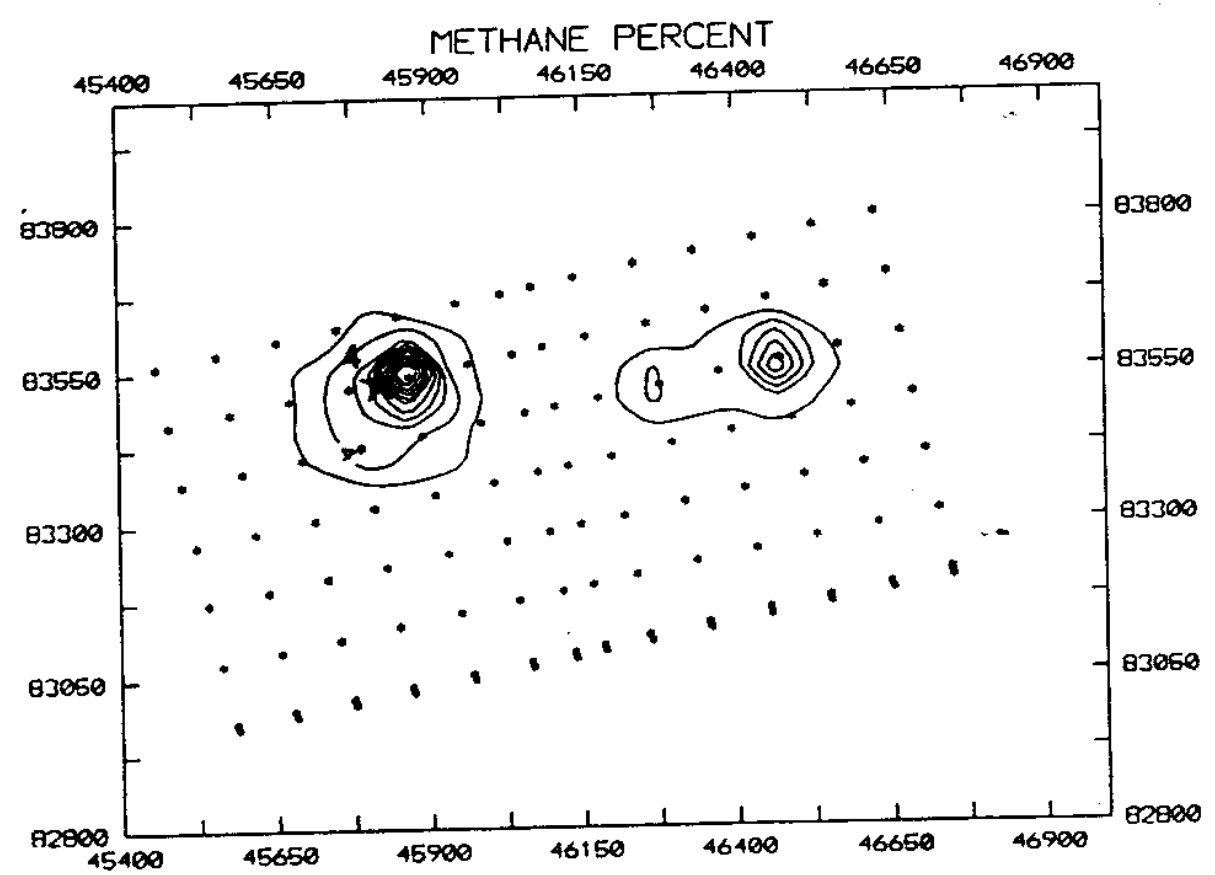

Figure 3. Soil Gas Percent Methane at the 740-G Landfill with Locations of the Groundsheets Indicated by 
File $=C: \backslash C P \backslash$ data2\GS3.10R Date printed $=03-27-1991$ Time $=15: 52: 48$ 0.00 to $4.50 \mathrm{~min}$. Low $Y=0.09991 \mathrm{mV}$ High $Y=1.15534 \mathrm{mv} \quad$ Span $=1.05543 \mathrm{mV}$

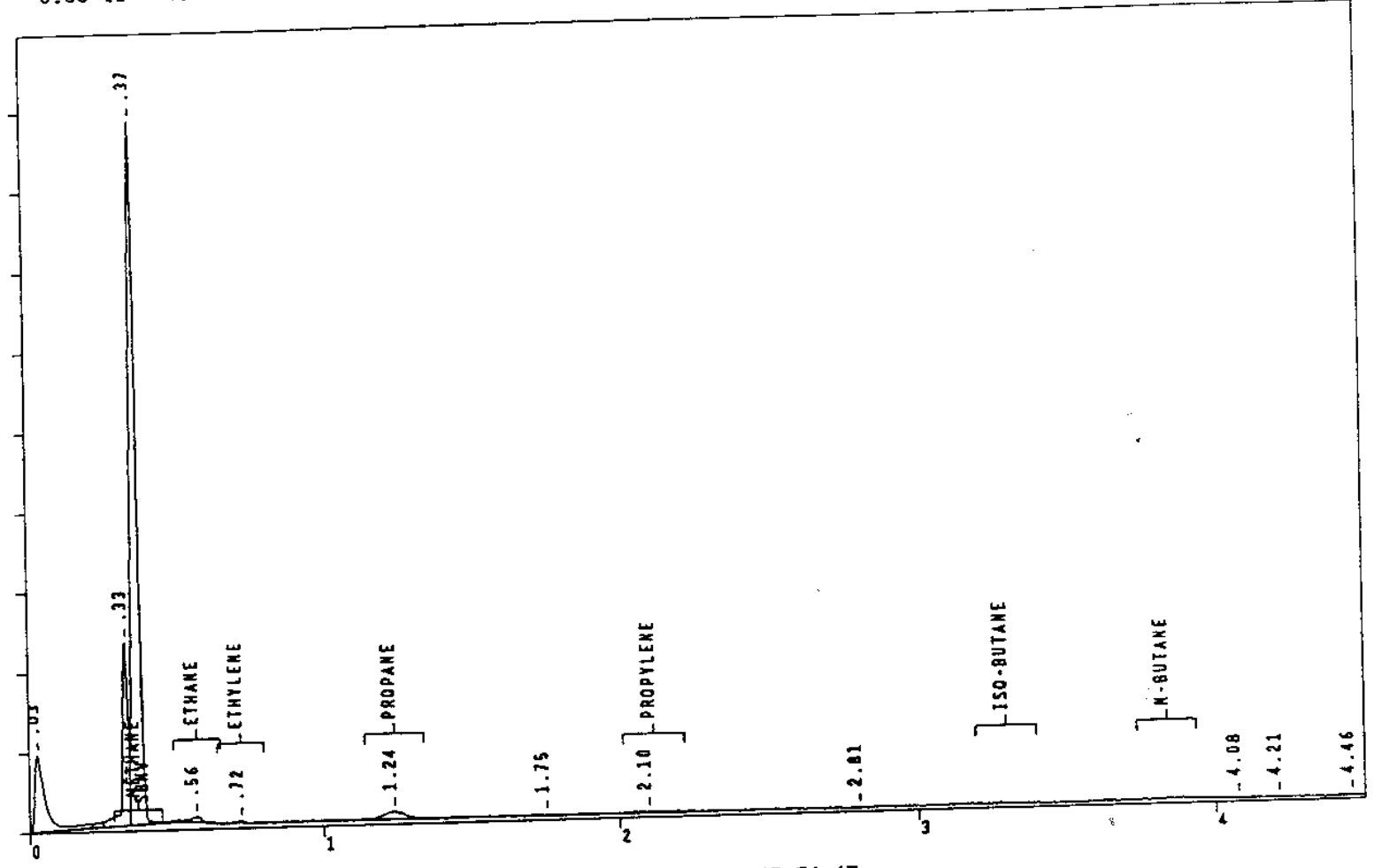

File=C: ICP \data2 $\backslash G S 3.10 R$ Date printed $=03-27-1991$ Time $=15: 54: 43$
0.00 to $4.49 \mathrm{~min}$. Lou $Y=$
$0.09991 \mathrm{mv}$ High $Y=$
$0.17306 \mathrm{mv}$
Span $=$
$0.07315 \mathrm{rN}$

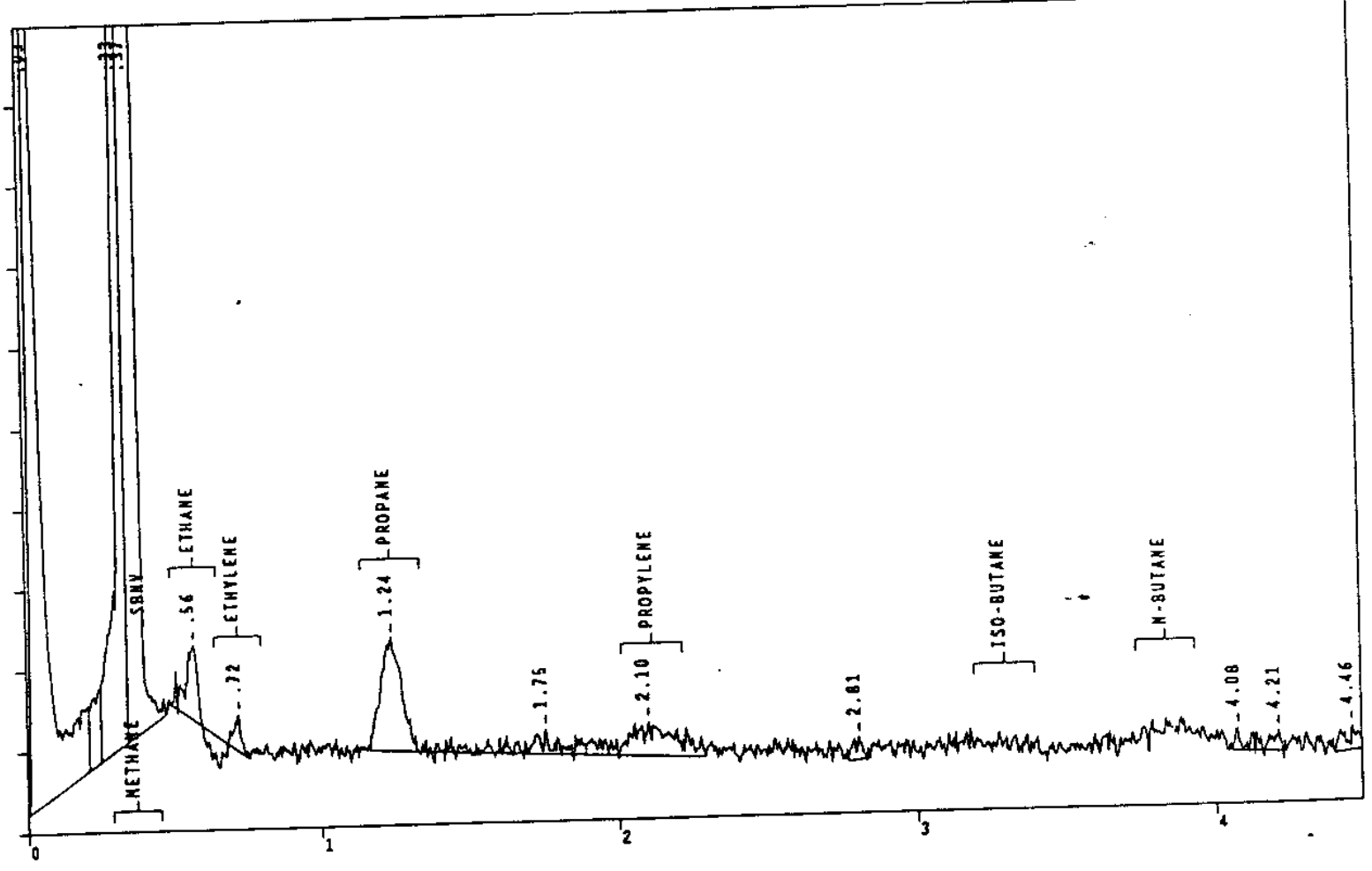

Eigure

4. Representative Chromatogram of $\mathrm{Cl-C4}$ Hydrocarbons Under Groundsheet \#1. 
File=C: \CP \data2\GS3.11R Date printed $=03-27-1991$ Time $=15: 45: 34$

0.00 to $4.50 \mathrm{~min}$. Low $Y=-0.05679 \mathrm{mv} H \mathrm{High} Y=43.68425 \mathrm{mv} \quad$ Span $=43.74104 \mathrm{mV}$

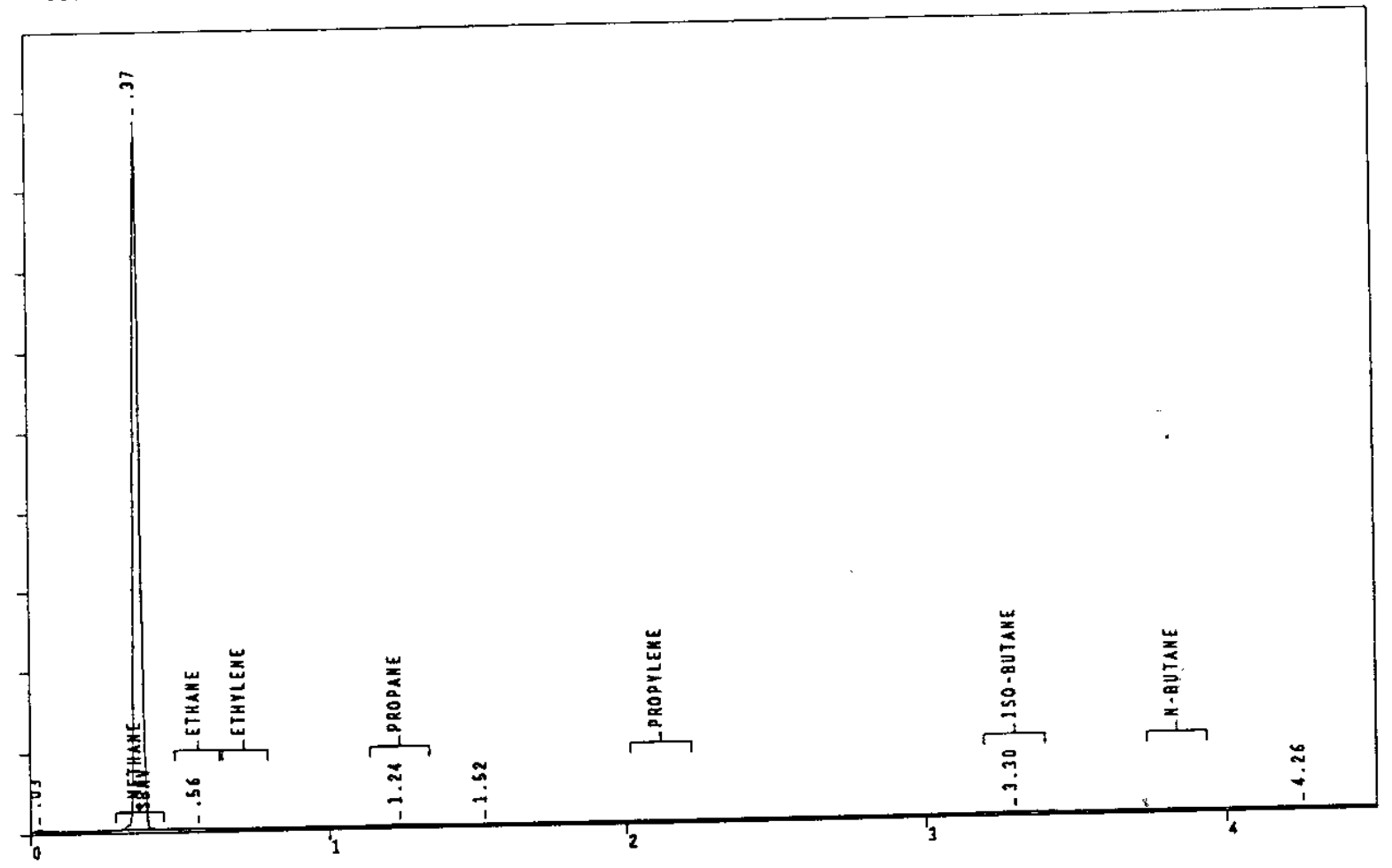

File=C: \CP \dataZ\GS3.11R Date printed $=03-27-1991$ Time $=15: 47: 36$
0.01 to $4.47 \mathrm{~min}$. Low $Y=$
$0.09445 \mathrm{un}$
High $Y=$
$0.21801 \mathrm{mv}$
Span $=$
$0.12357 \mathrm{mv}$

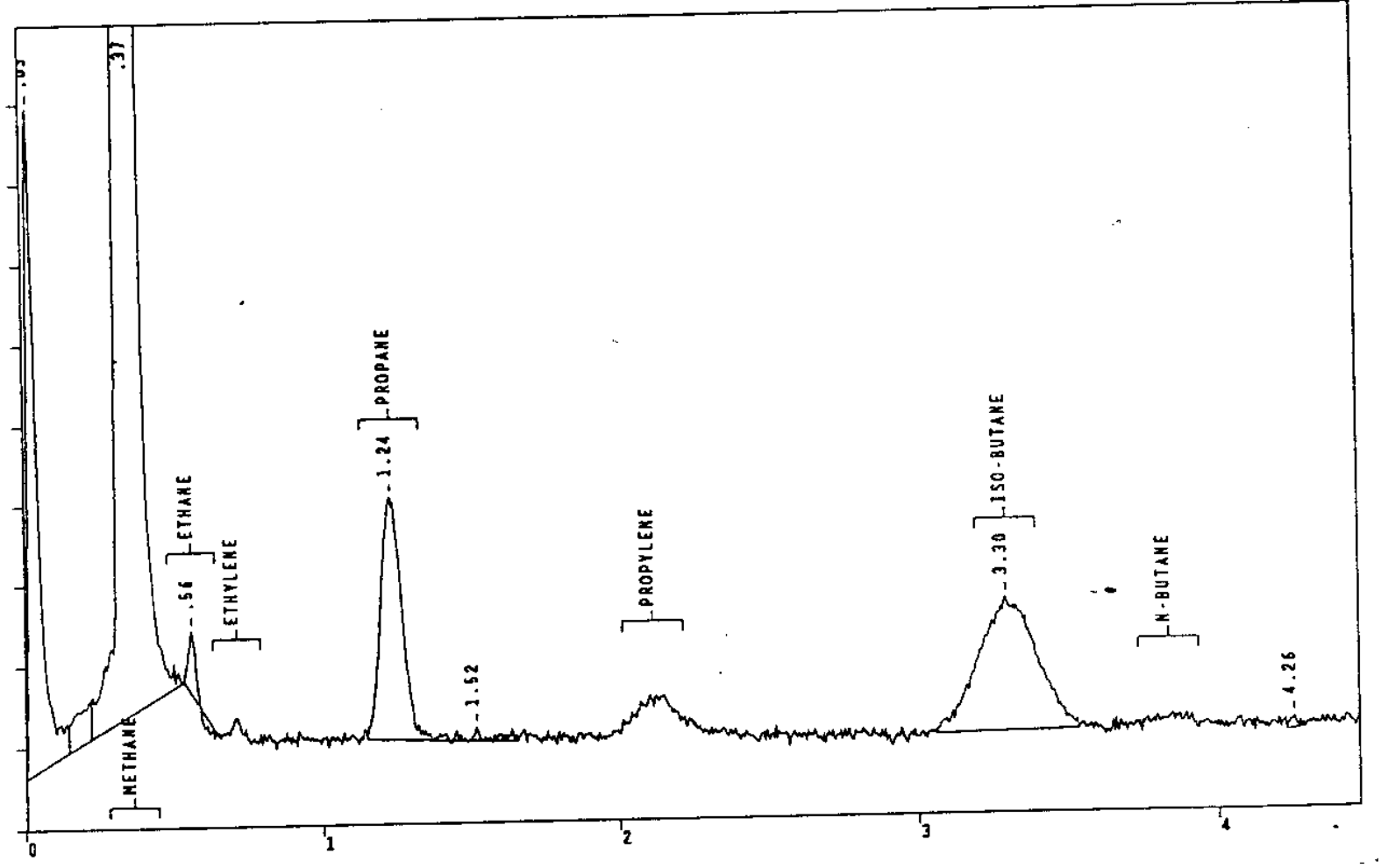

Figure 5. Representative Chromatogram of $\mathrm{Cl}-\mathrm{C} 4$ Hydrocarbons Under Groundsheet \#2. 
File $=C: \backslash C P \backslash$ data2 $\backslash G S 3.13 R$ Date printed $=03-27-1991$ Time $=16: 05: 06$ $4.50 \mathrm{~min}$. Low $Y=0.10376 \mathrm{mv}$ High $Y=0.99591 \mathrm{mv}$ Span $=0.89215 \mathrm{mv}$

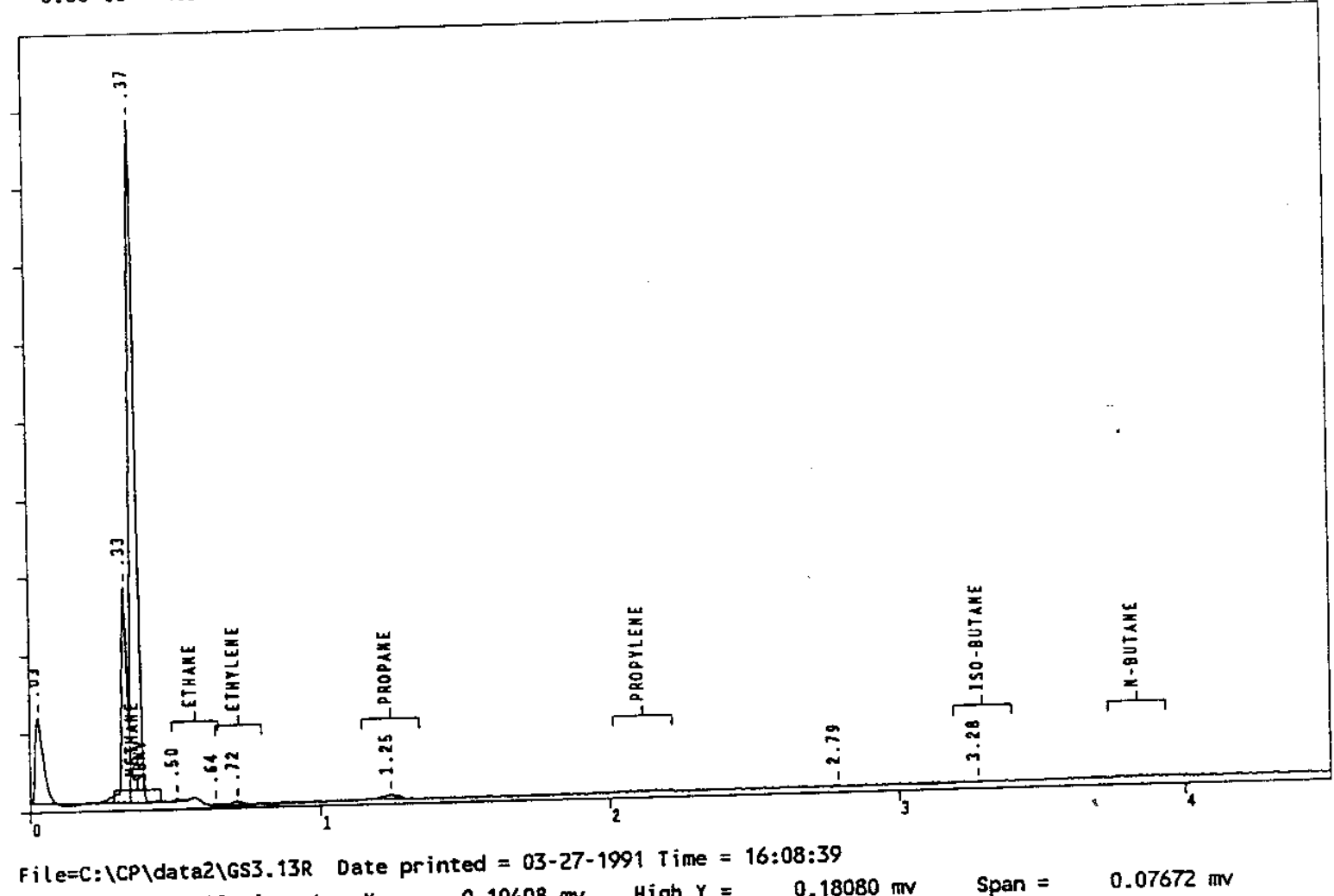
File=C:\CP\data2\GS3.13R Date printed $=03-27-1991$ Time $=16: 08: 39$
0.01 to $4.48 \mathrm{~min}$. Low $Y=0.10408 \mathrm{mv}$ High $Y=0.18080 \mathrm{mv} \quad$ Span $=$

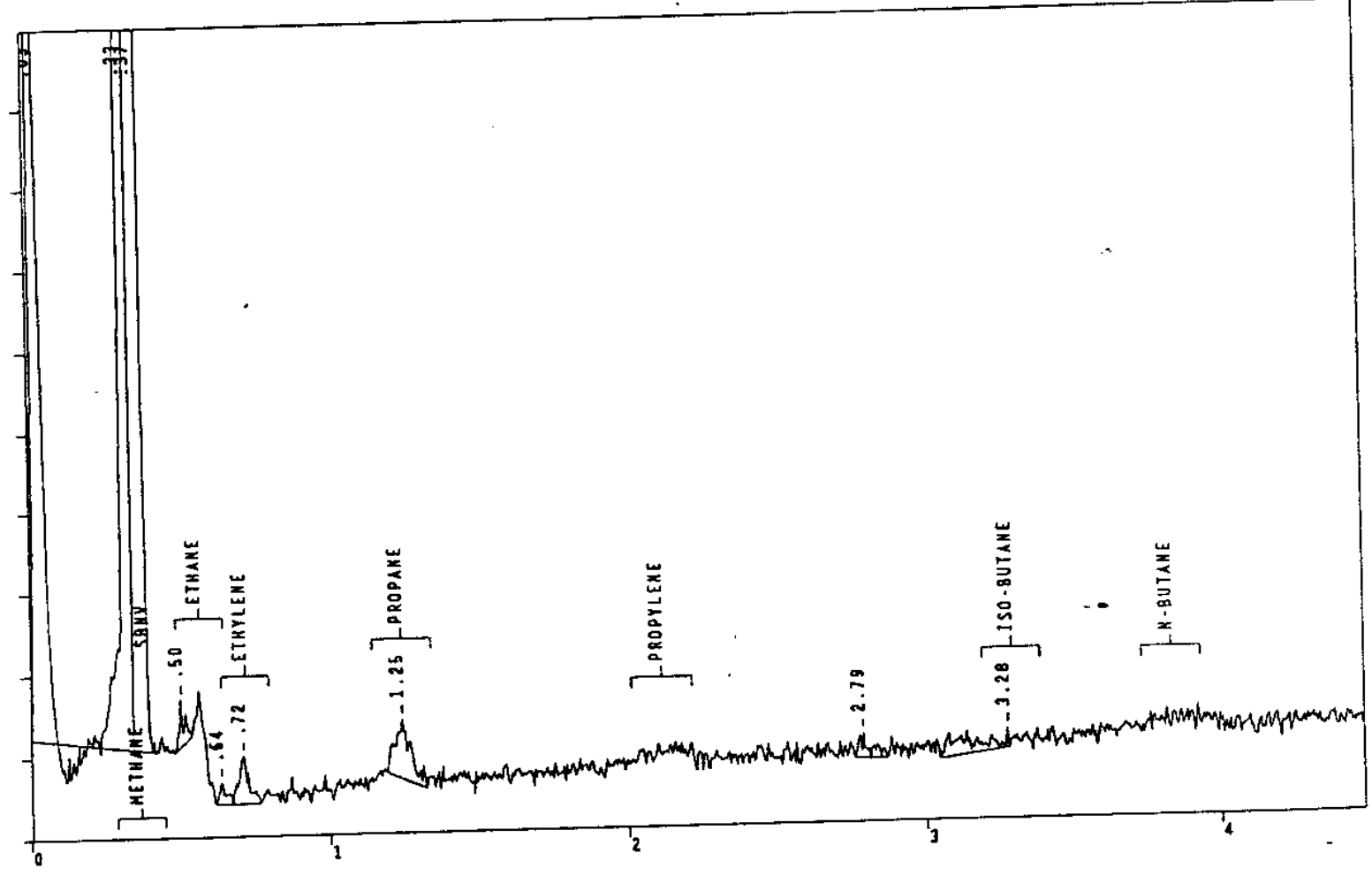

Figure 6. Representative Chromatogram of C1-C4 Hydrocarbons Under Groundsheet \#3. 
File=C: $\backslash C P \backslash$ data2 $\backslash G S 3.12 R$ Date printed $=03-27-1991$ Time $=15: 58: 07$
0.00 to $4.50 \mathrm{~min}$. Low $Y=$
$0.10310 \mathrm{mv}$
High $Y=$
$1.00218 \mathrm{mV}$
Span $=$
$0.89908 \mathrm{mv}$

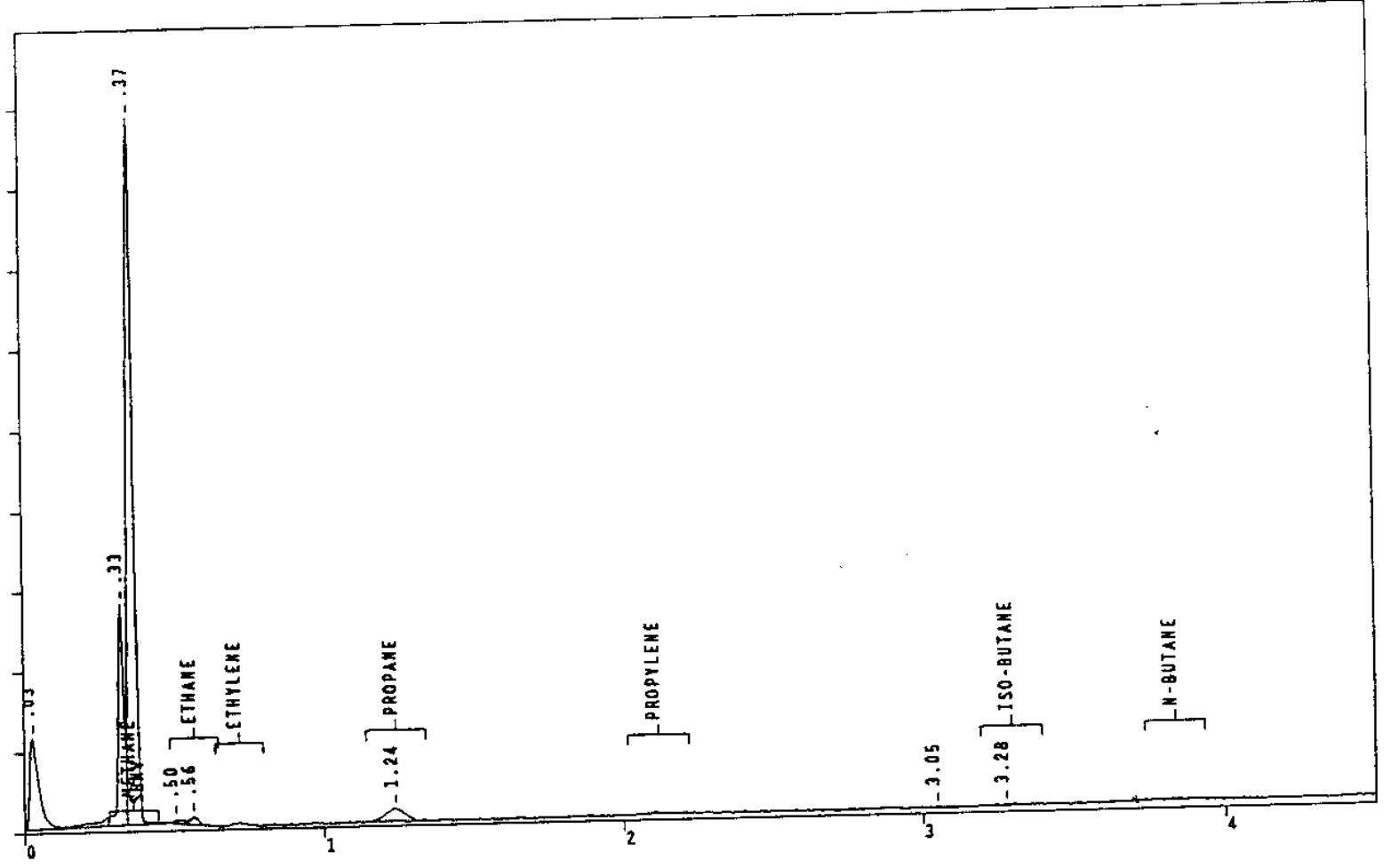

File=C: \CP\data2\GS3.12R Date printed $=03-27-1991$ Time $=15: 59: 39$
0.00 to 4.50 min. Low $Y=$
$0.10310 \mathrm{mv}$
High $Y=$
$0.17432 \mathrm{mv}$
Span $=$
$0.07121 \mathrm{mv}$

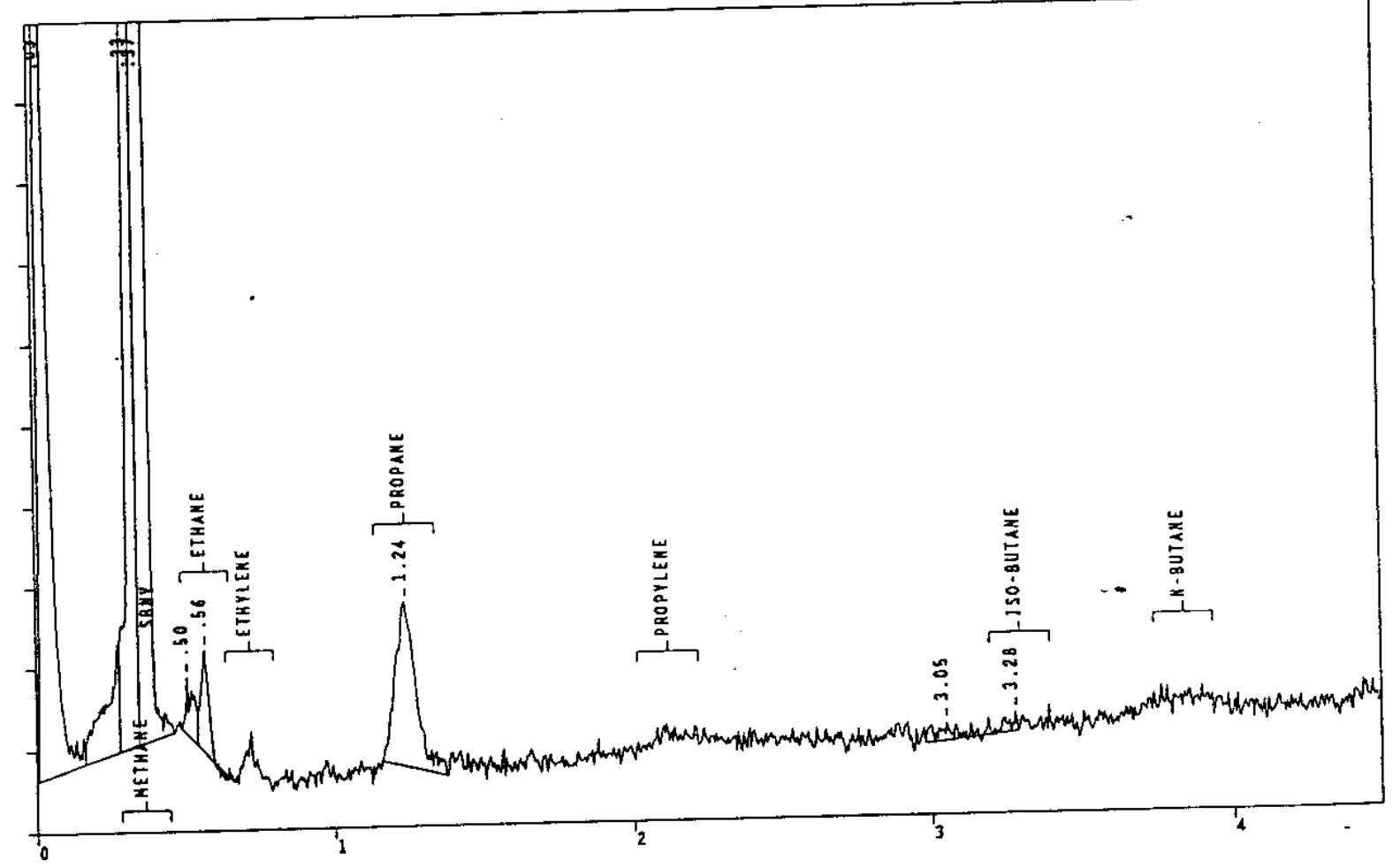

Figure 7. Representative Chromatogram of $\mathrm{Cl}-\mathrm{C} 4$ Hydrocarbons Under Groundsheet \#4. 
File=C: \CP \data2\GS2.15R Date printed $=03-27-1991 \mathrm{Time}=15: 10: 07$
0.00 to $4.49 \mathrm{~min}$. Low $Y=$

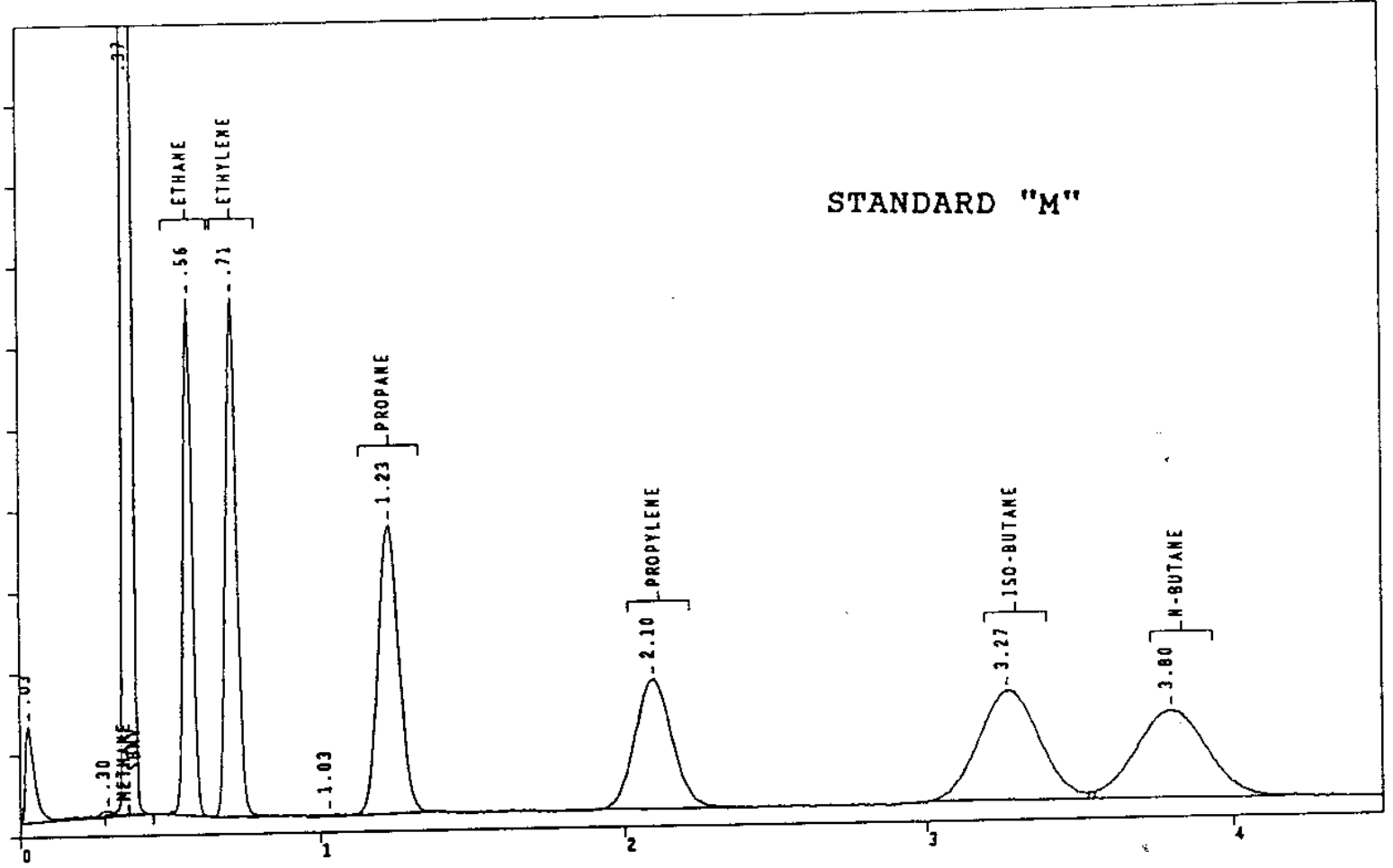

File=c:ICPIdata2\GS4.SOR Date printed $=03-27-1991 \mathrm{Time}=14: 39: 42$ 0.00 to

Low $Y=$

$-0.39282 \pi \mathrm{n}$

High $\mathbf{Y}=$

$8.06816 \mathrm{mv}$

$\operatorname{Span}=$

$8.46098 \mathrm{mv}$

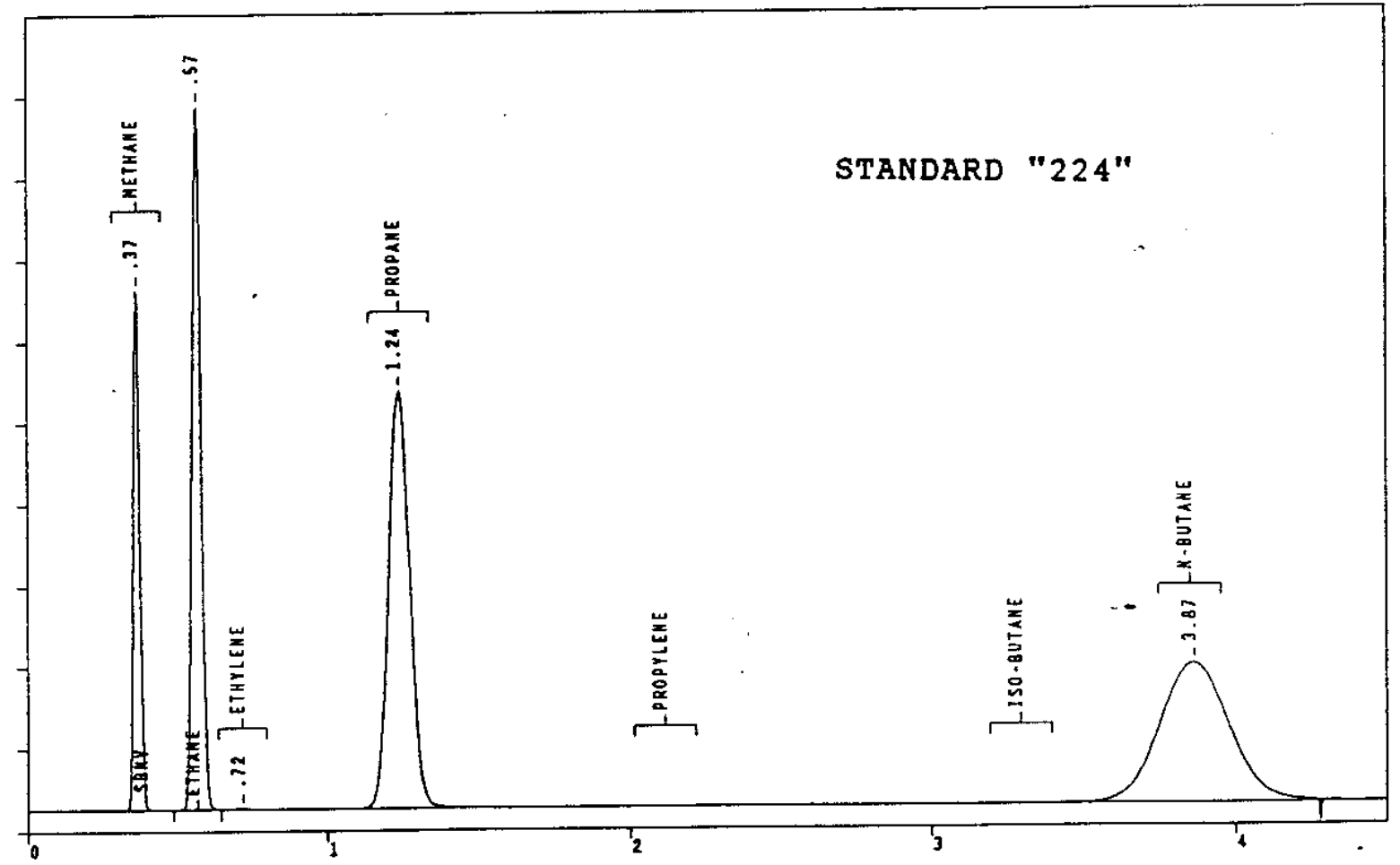

Figure 8. Representative Gas Chromatogram of $\mathrm{Cl}-\mathrm{C} 4$ Hydrocarbon standard " $M$ " and Standard "224". 


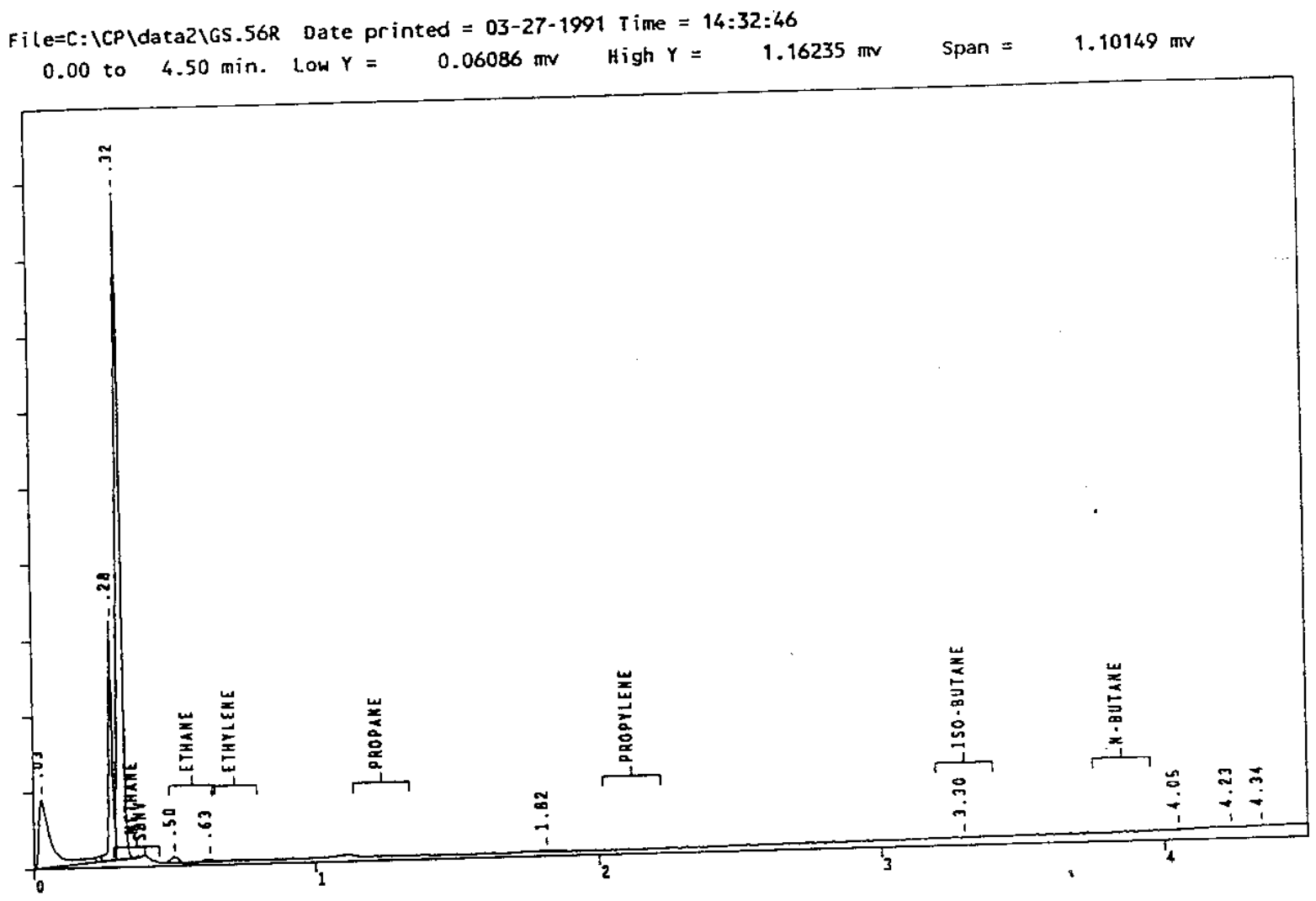

Figure 10. Representative Chromatogram of System Blank \#5 
File=C: ICP \data2\GS2.40R Date printed $=03-27-1991$ Time $=15: 26: 43$ 0.00 to $4.50 \mathrm{~min}$. Low $Y=0.11465 \mathrm{mv}$ High $Y=$

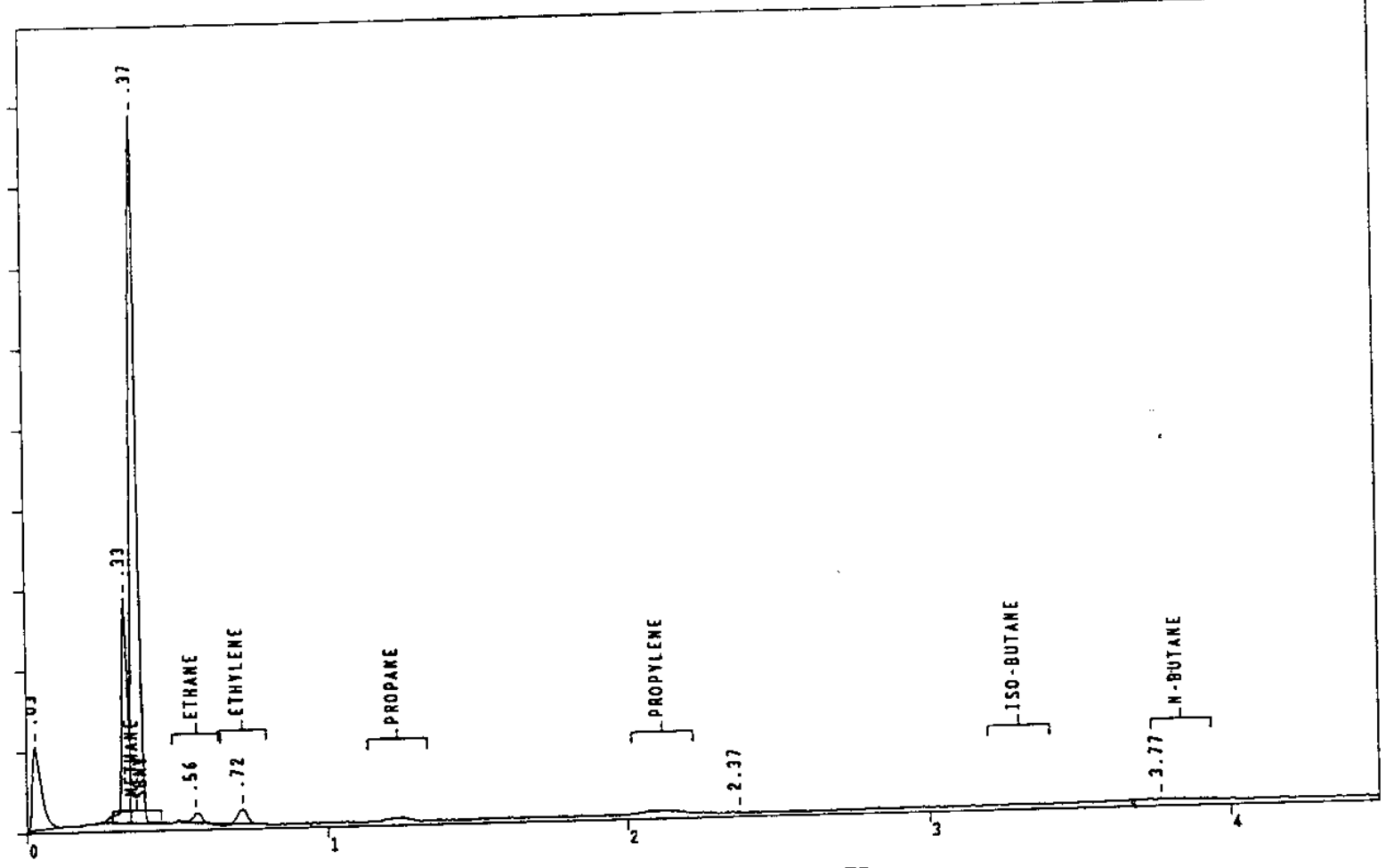

File=C:ICP\data2 \GS2.40R Date printed $=03-27-1991$ Time $=15: 29: 32$
0.01 to
4.49 min.
Low $Y=$
$0.11465 \mathrm{mv}$
High $Y=$
$0.22534 \mathrm{mN}$
Span $=$
$0.11069 \mathrm{mv}$

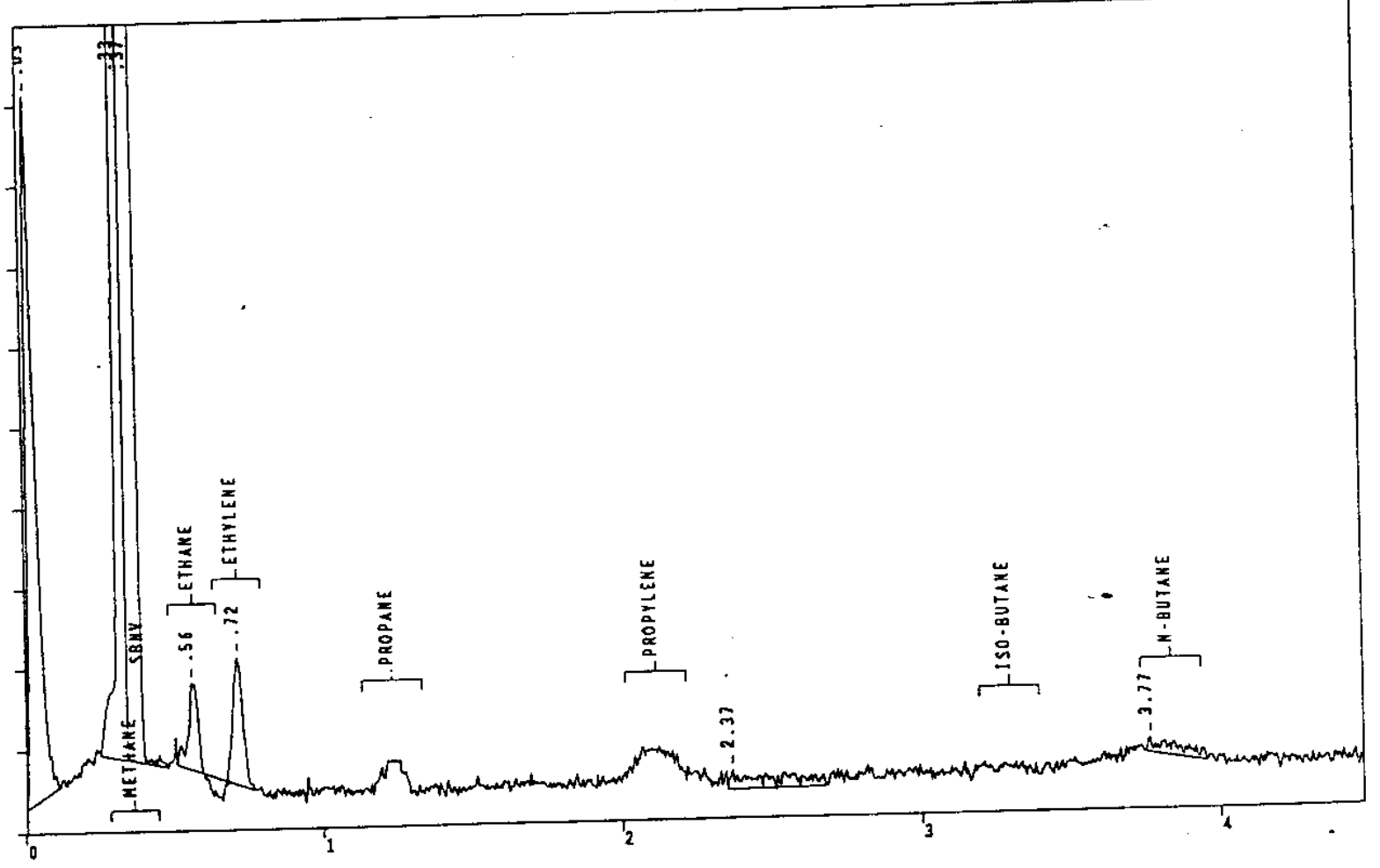

Figure 11. Representative Chromatogram of System Blank \#19 


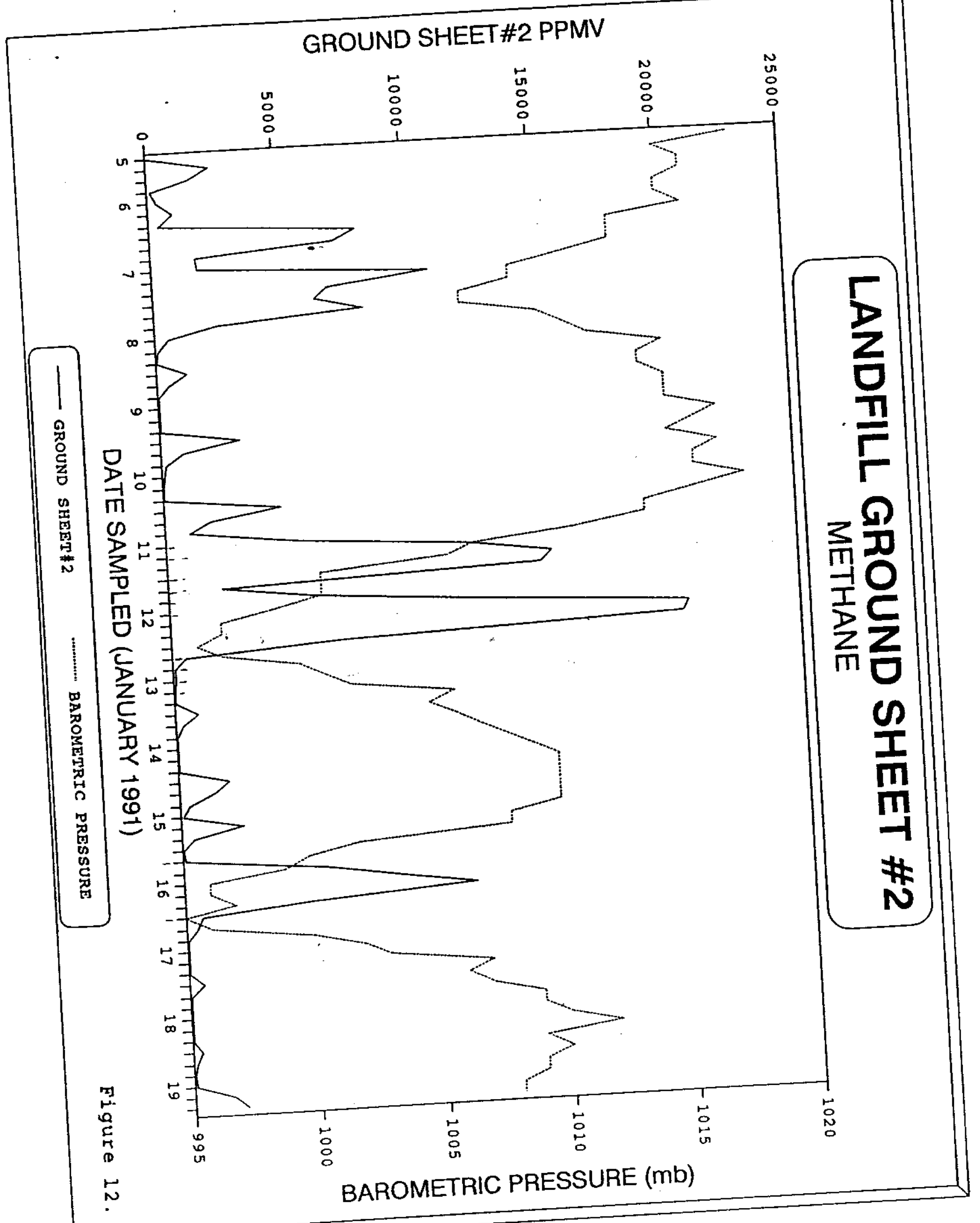




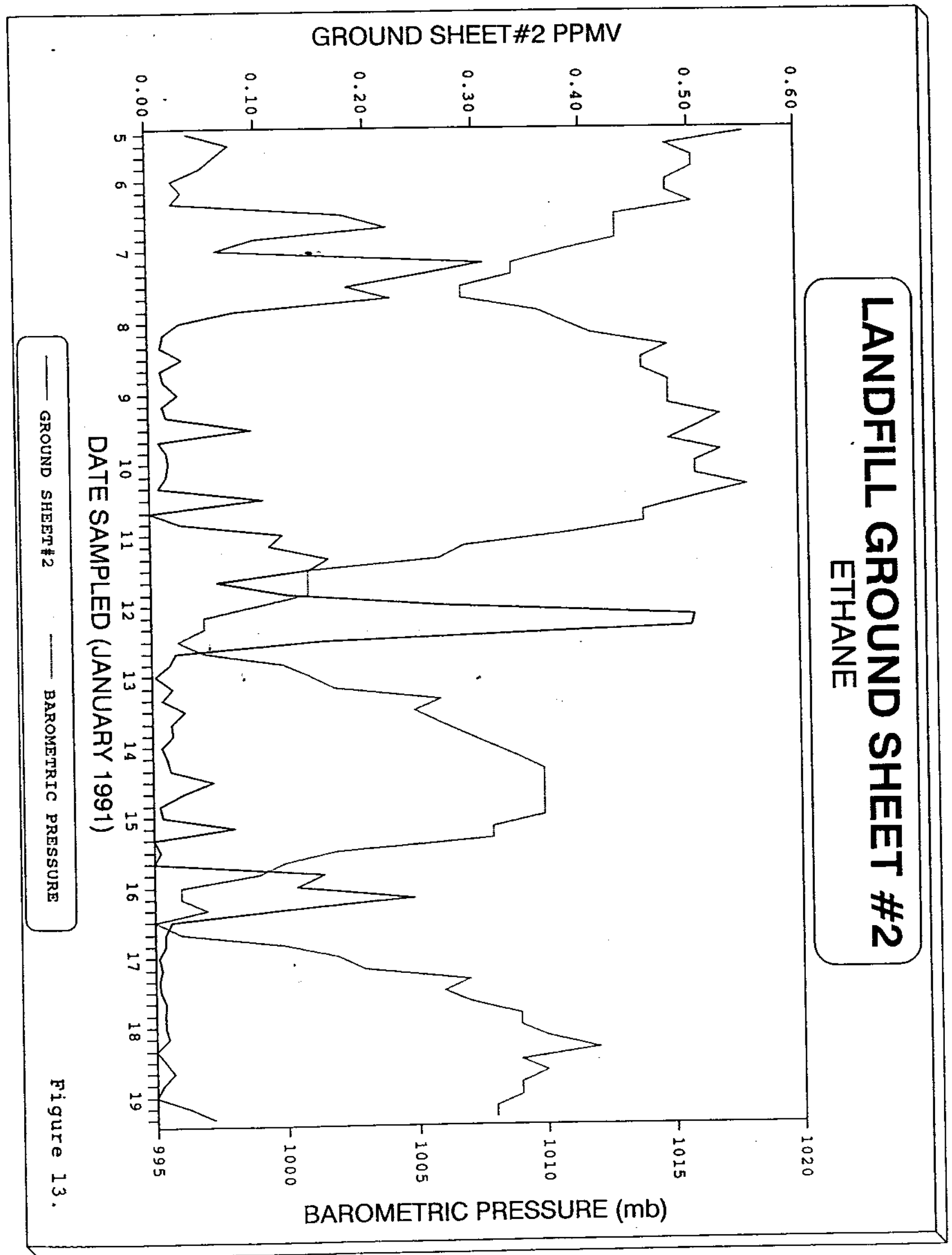




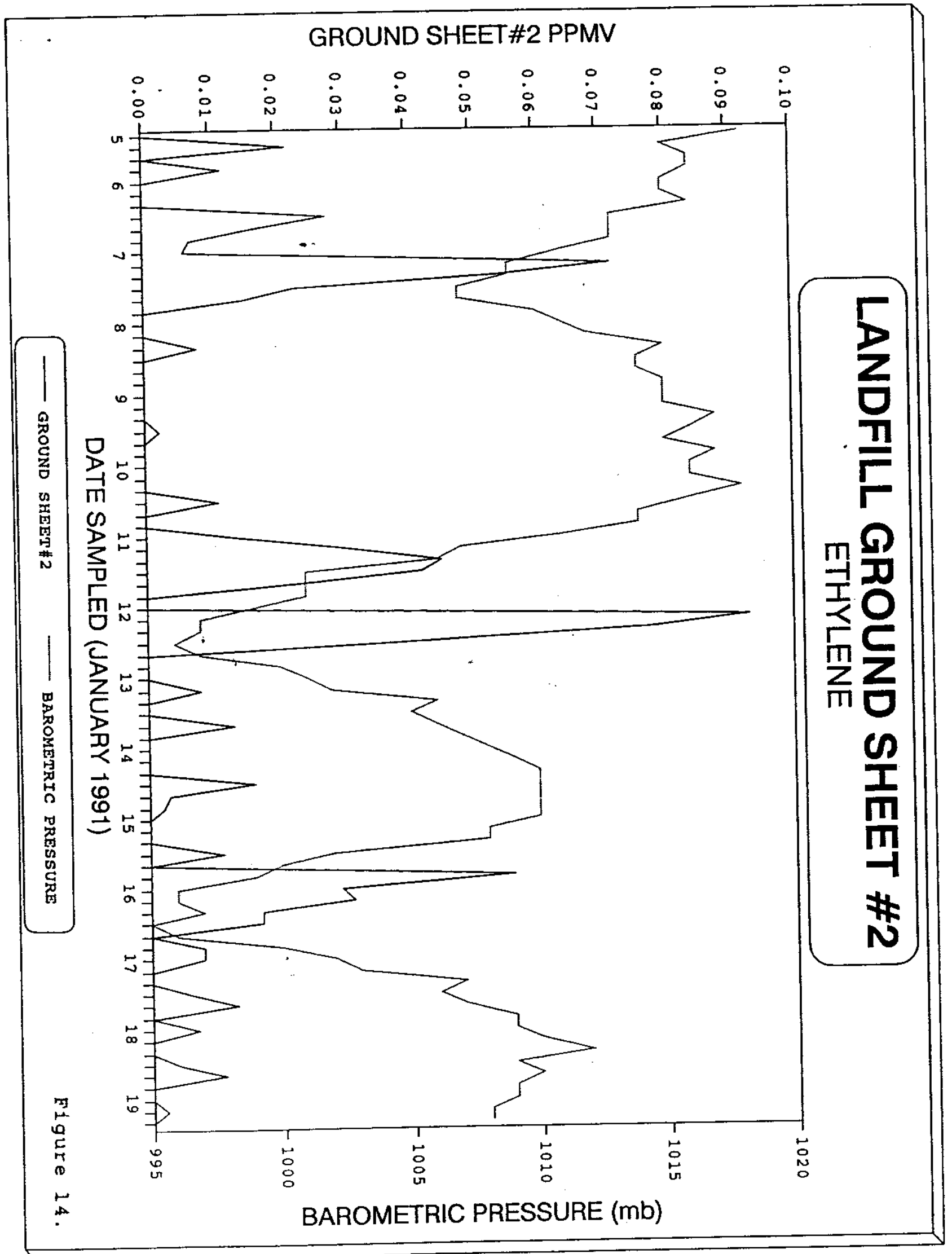




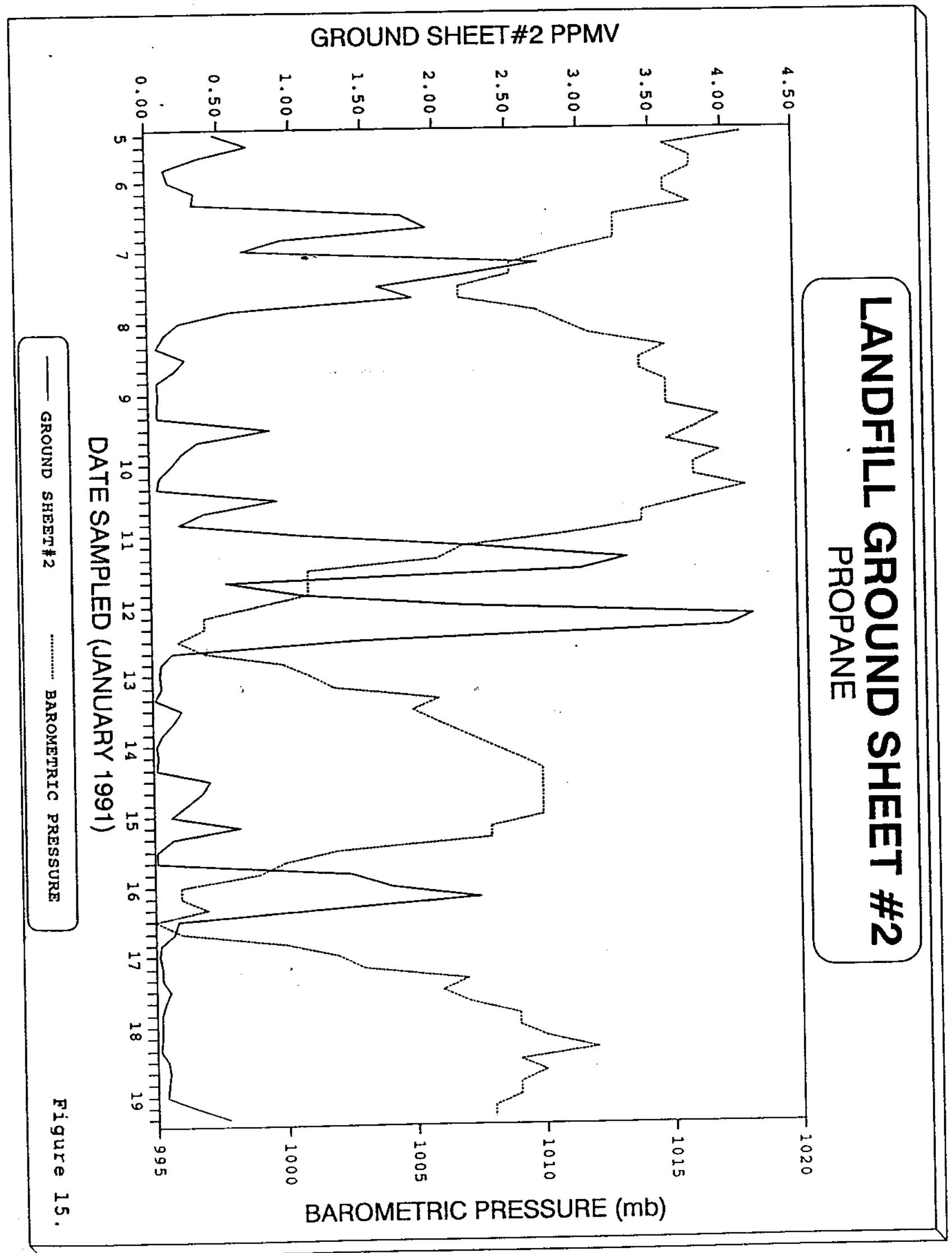




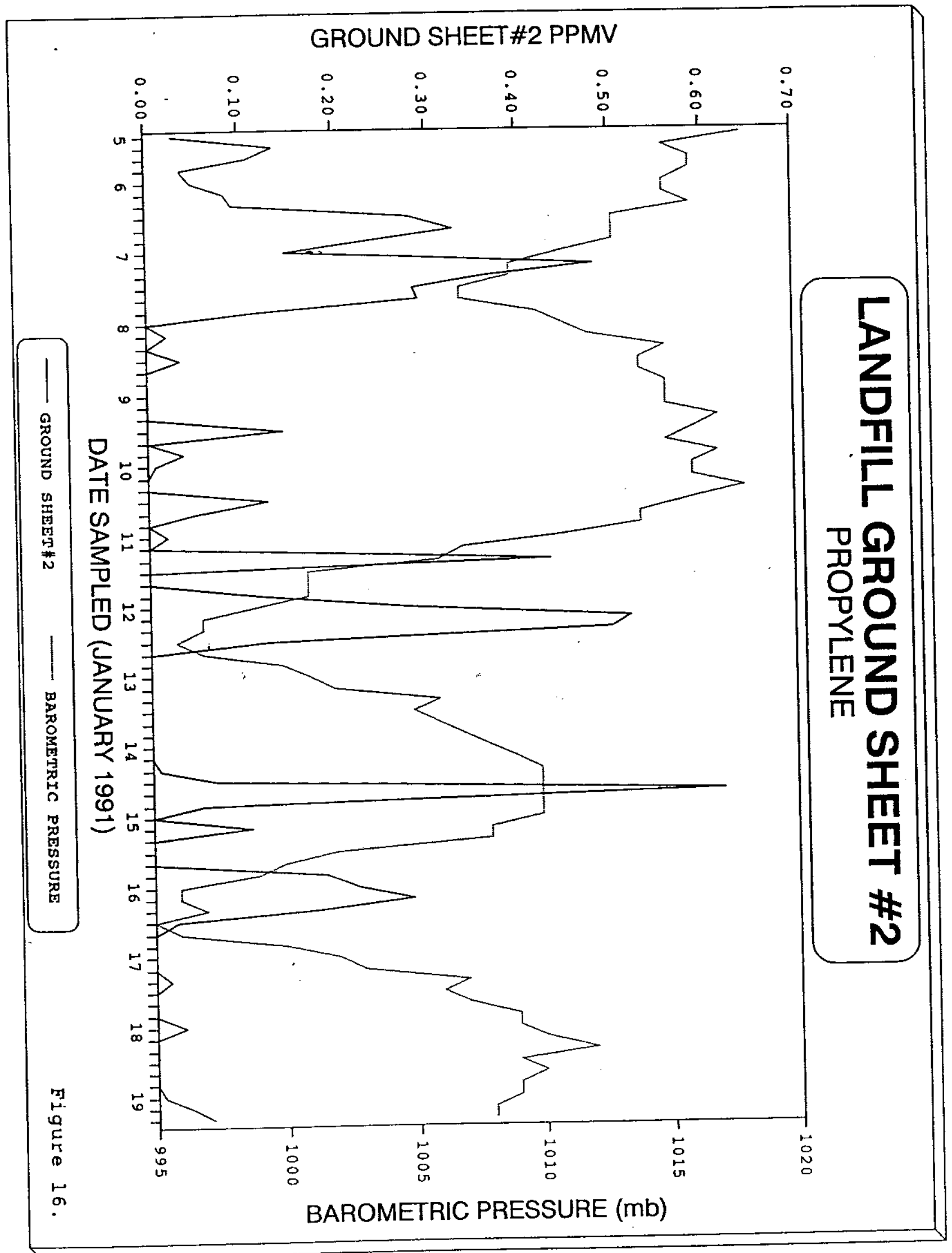




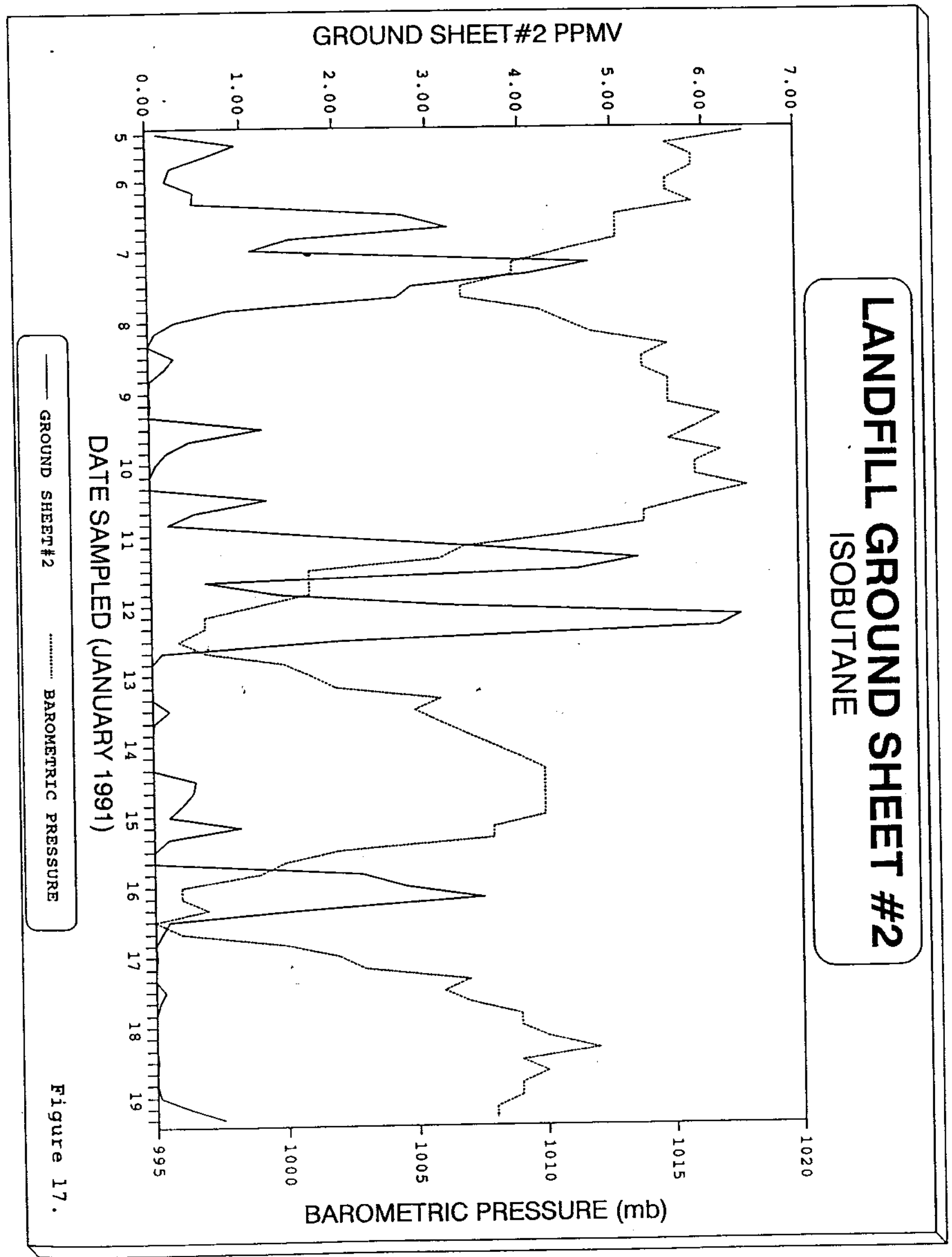




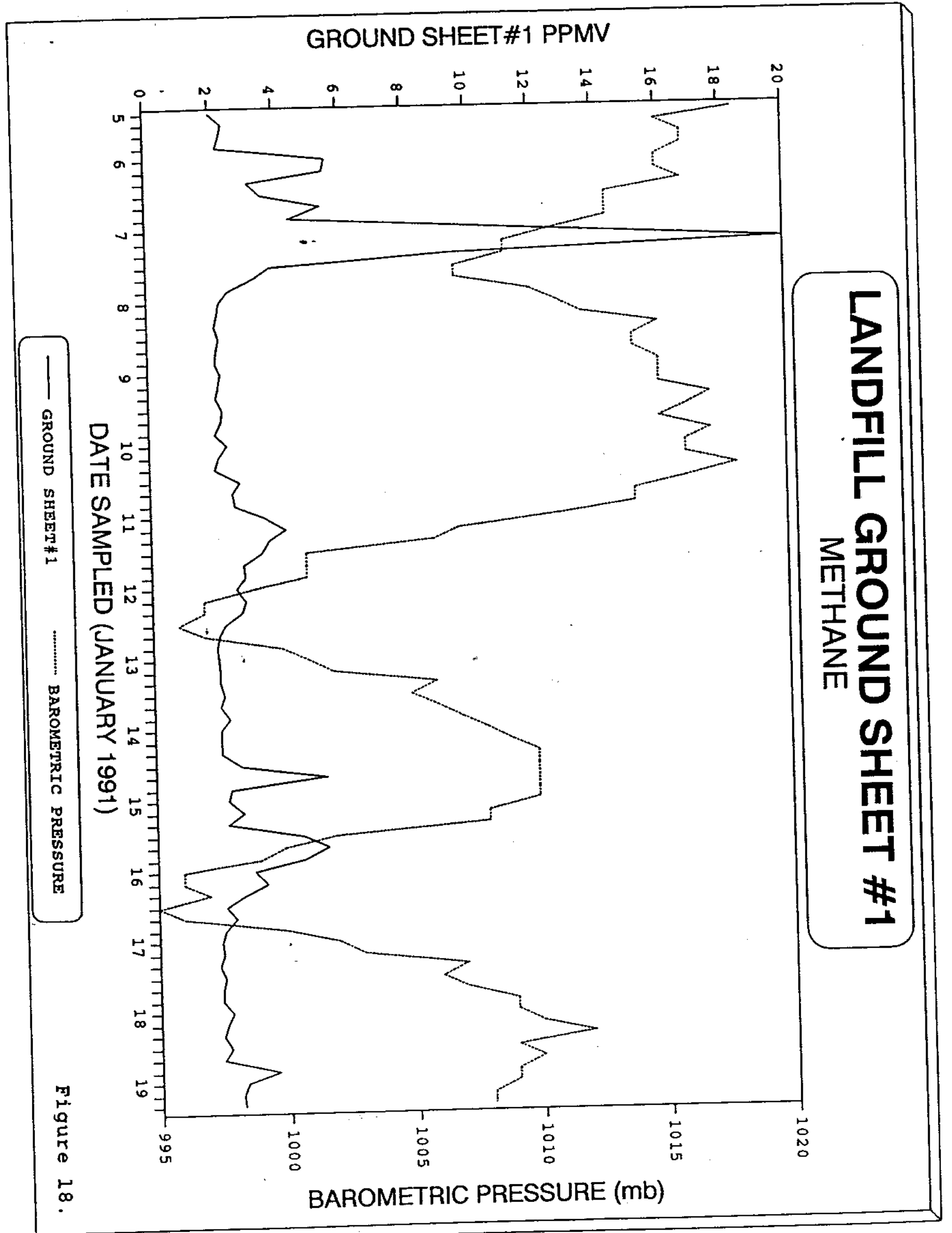




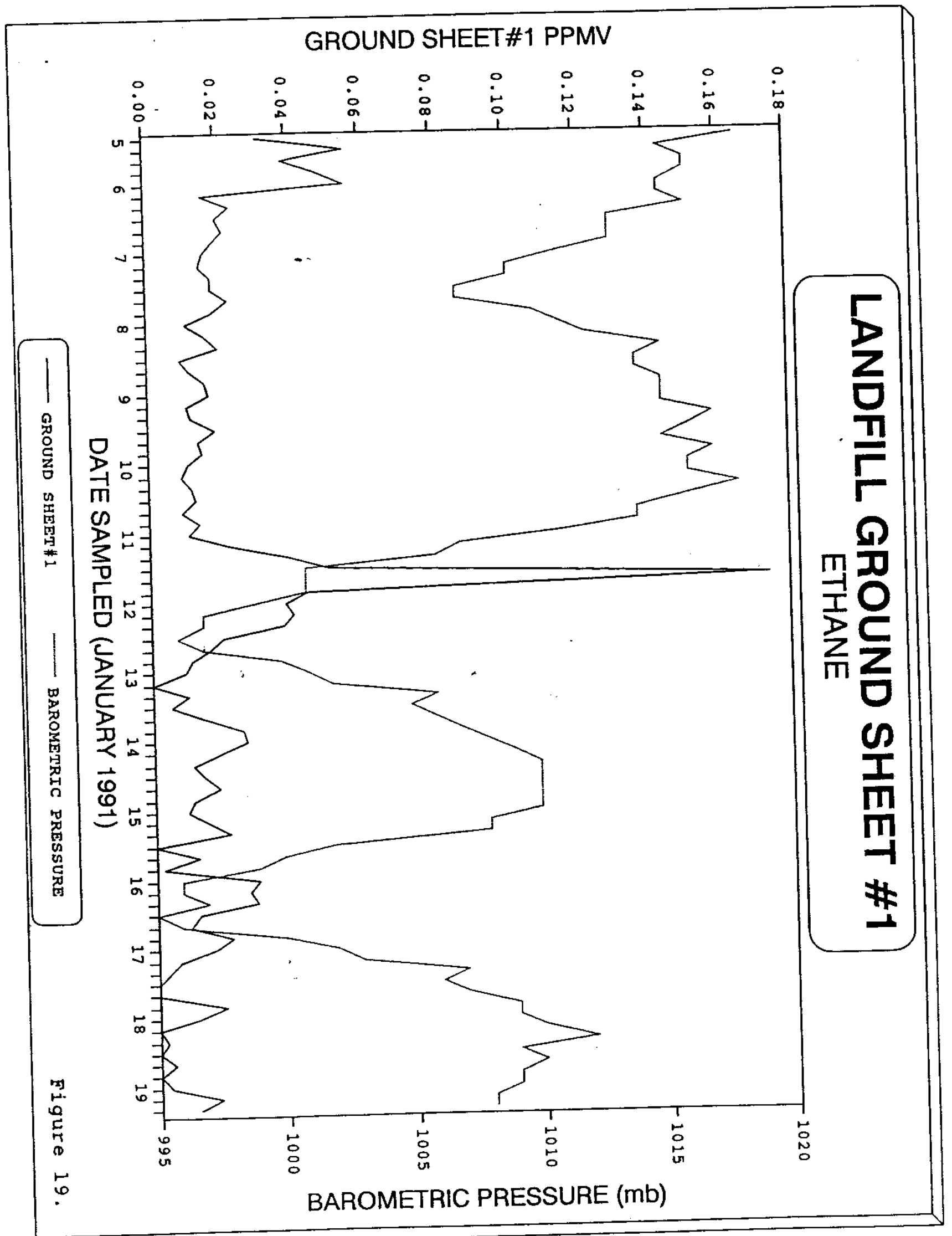




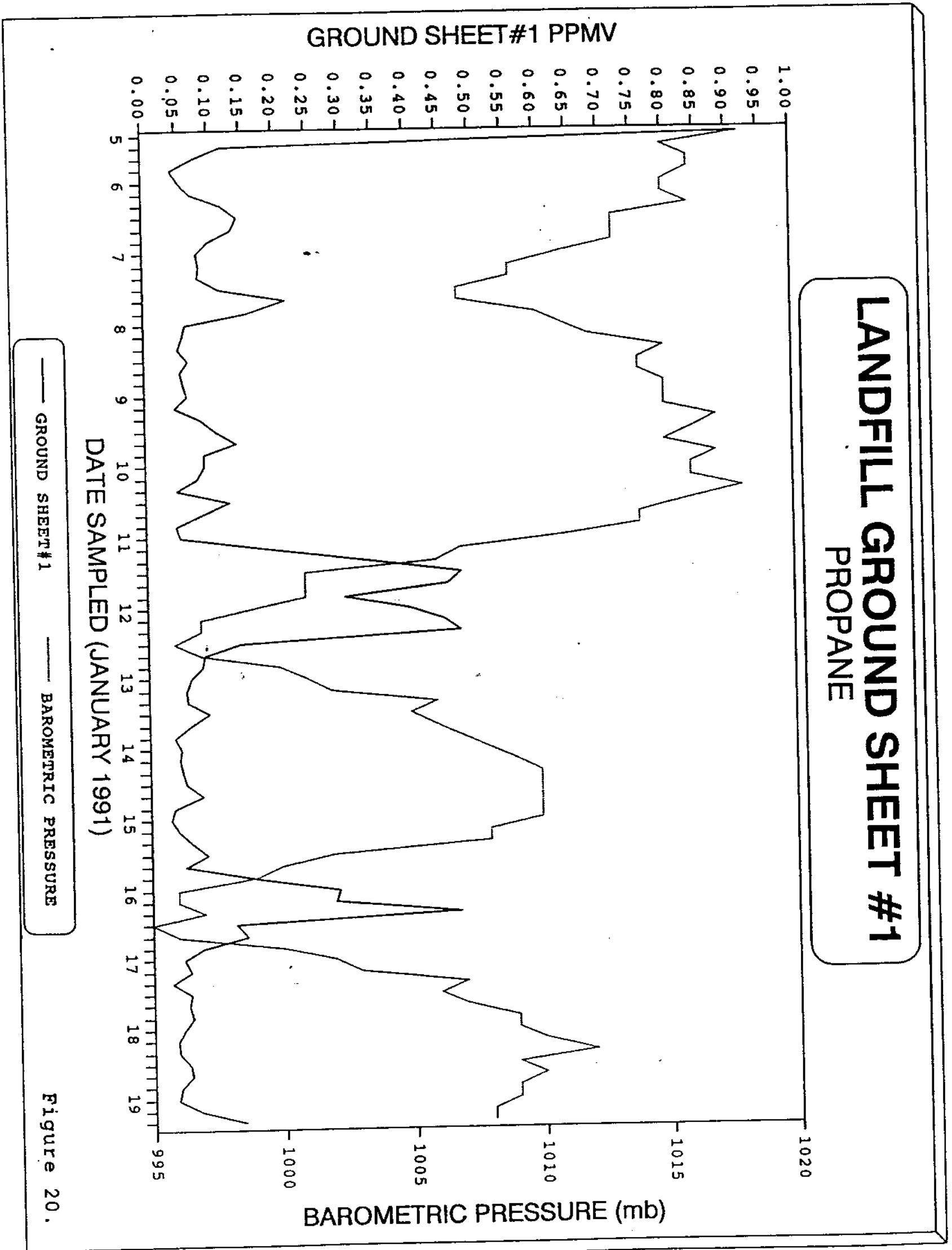




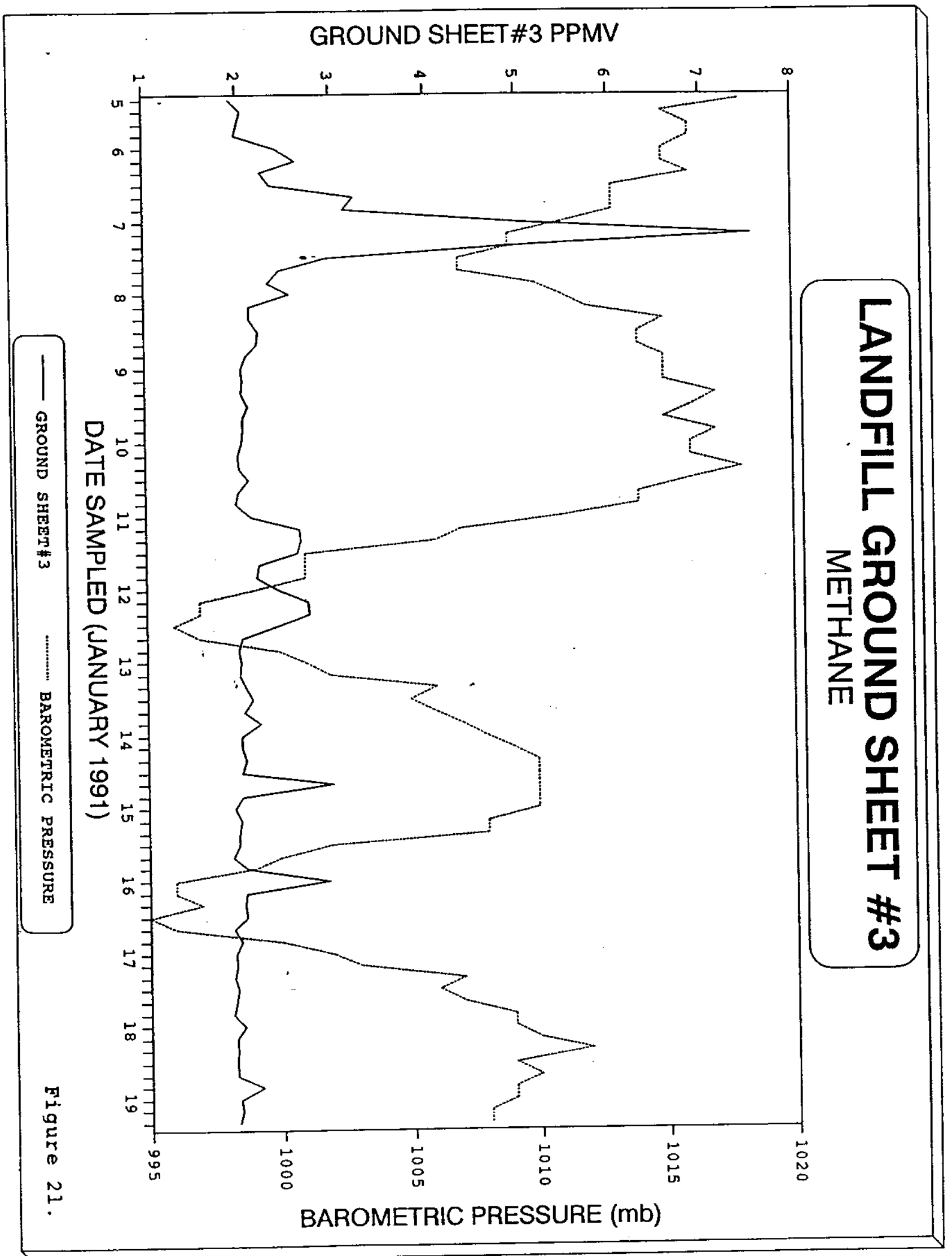




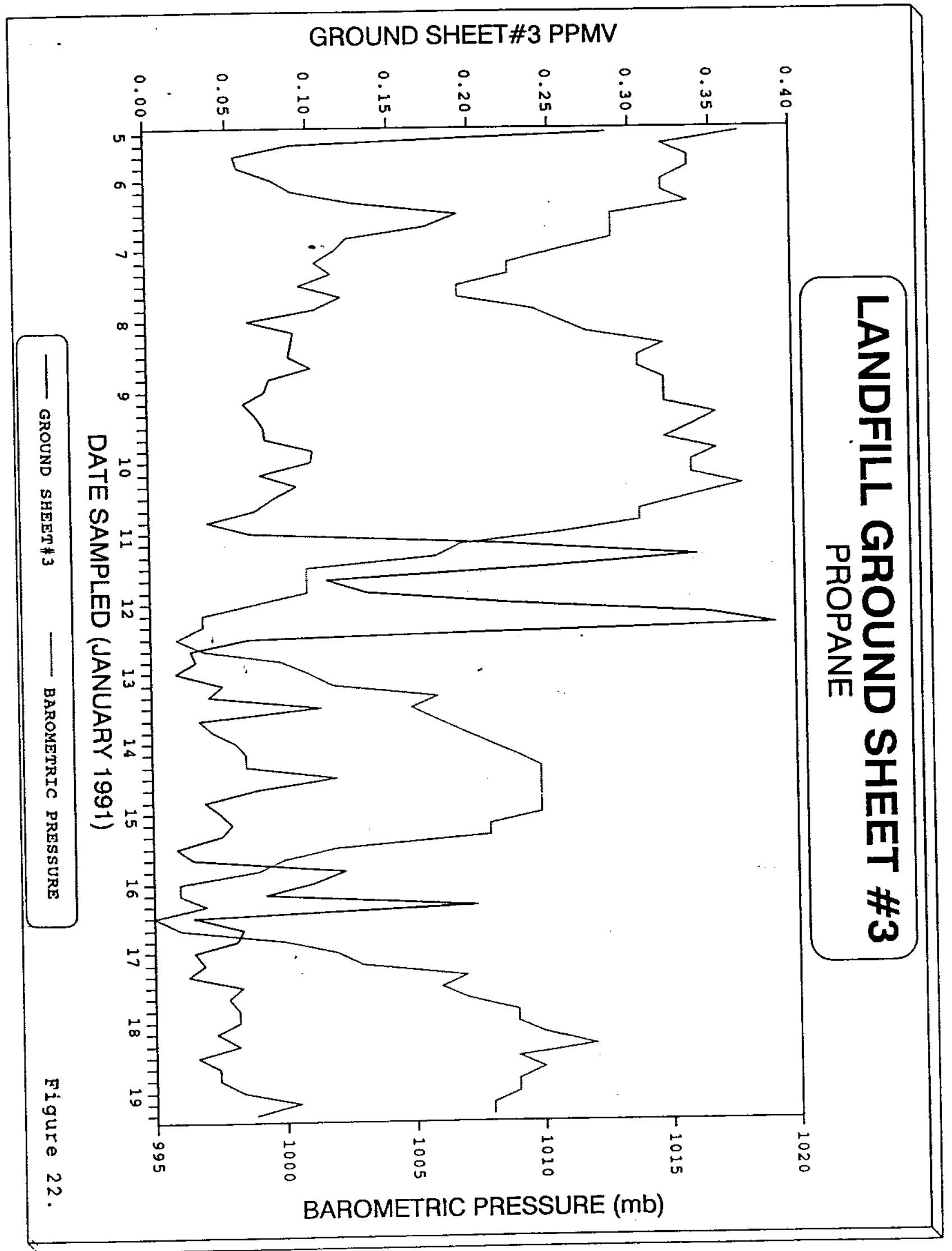




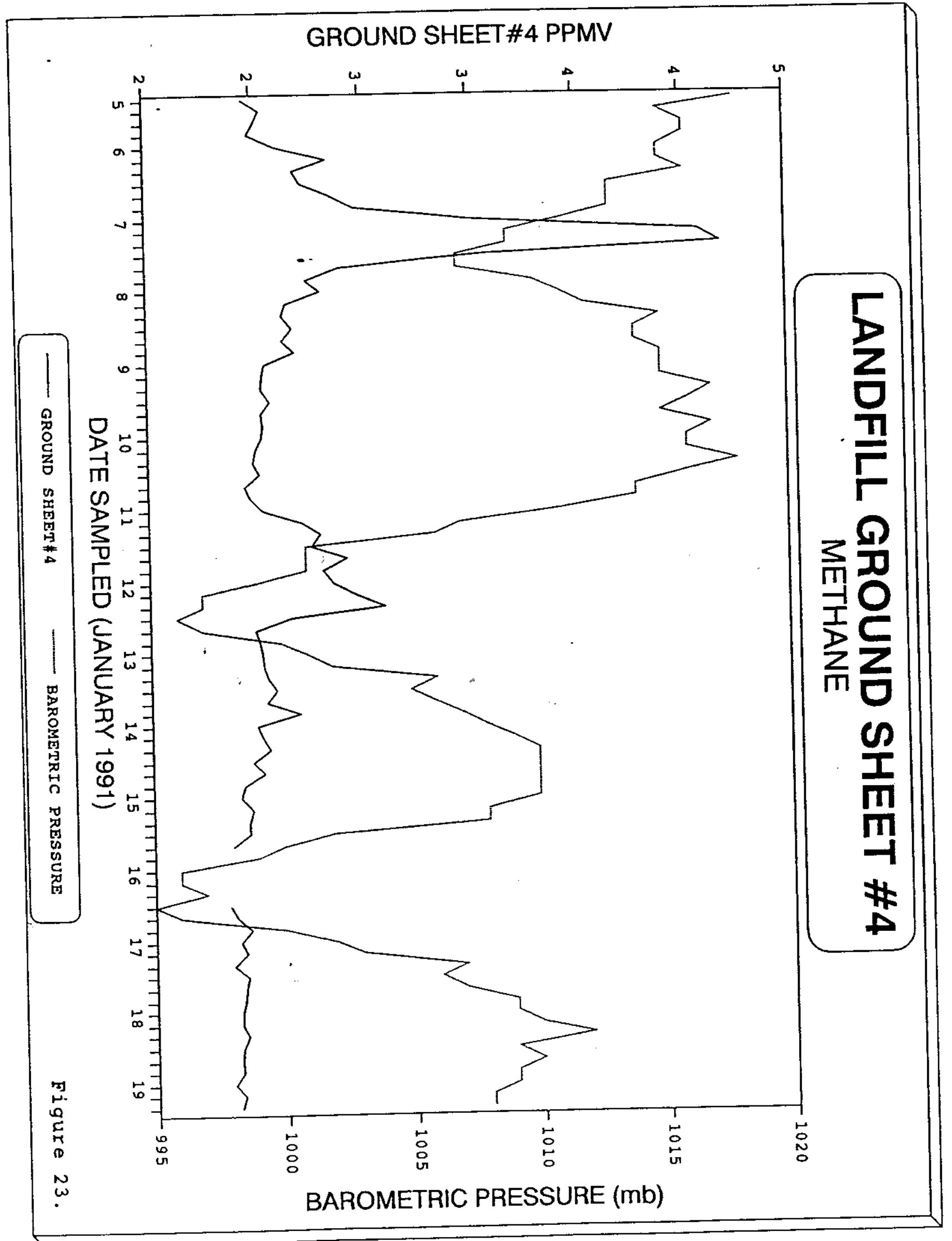




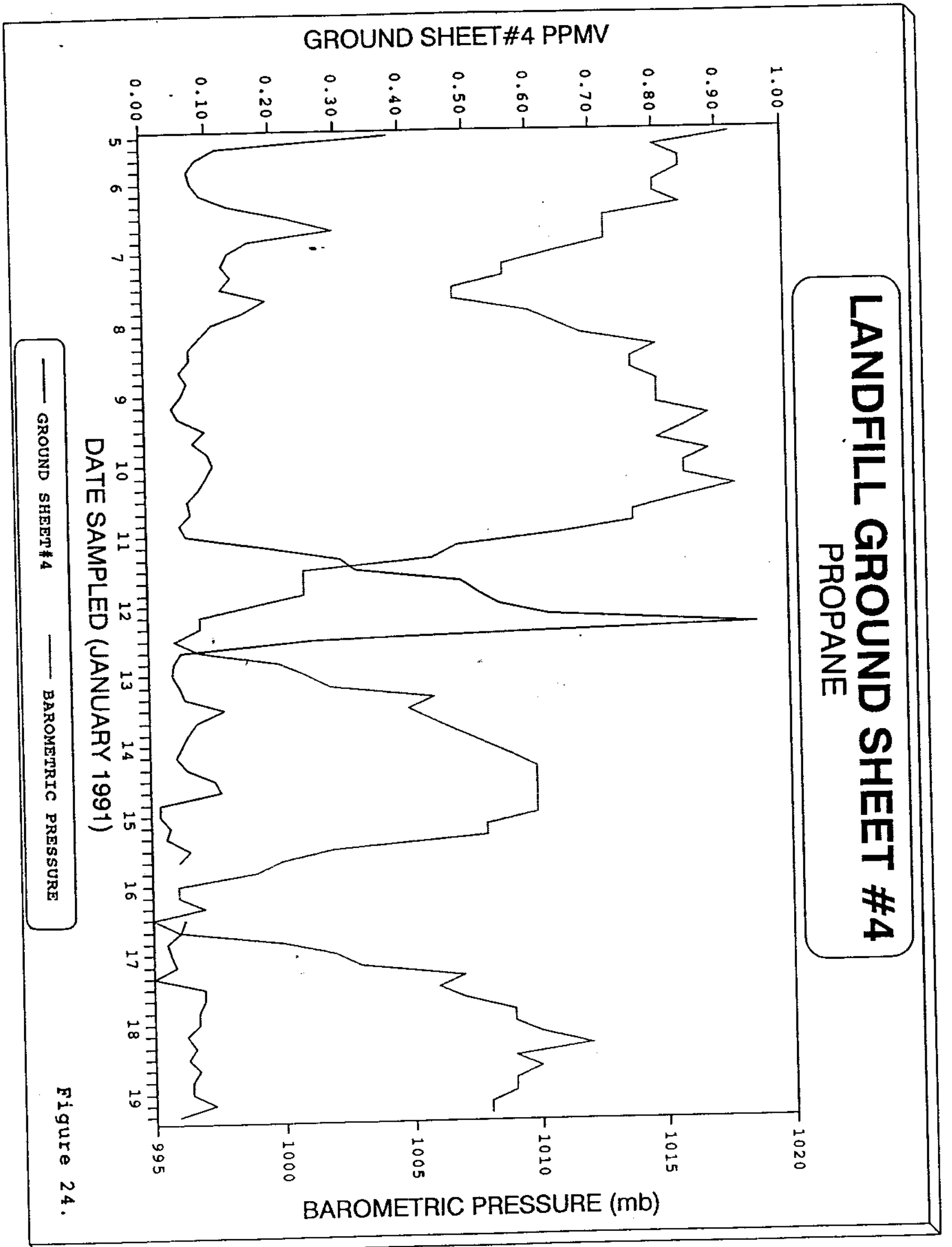


File=C:ICP\dSIUSA.36R Date printed $=03-27-1991$ Time $=16: 50: 45$

1.00 to $30.00 \mathrm{~min}$. Low $Y=-0.96881 \mathrm{mv}$ High $Y=247.15326 \mathrm{mV}$ Span $248.12207 \mathrm{mv}$

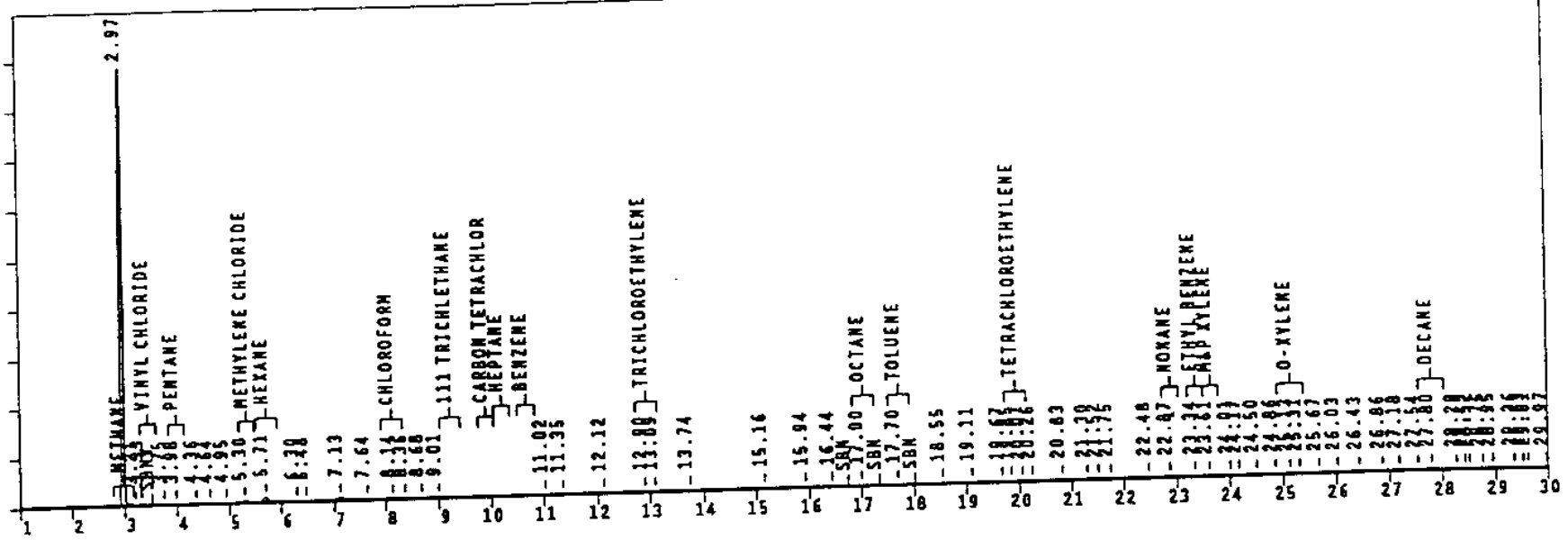

File $=C: \backslash C P \backslash$ S5 $W 5 A .36 R$ Date printed $=03-27-1991$ Time $=16: 51: 59$

3.70 to $30.00 \mathrm{~min}$. Low $Y=-0.05649 \mathrm{mV}$ High $Y=2.47513 \mathrm{mV}$ Span $=2.53163 \mathrm{mv}$

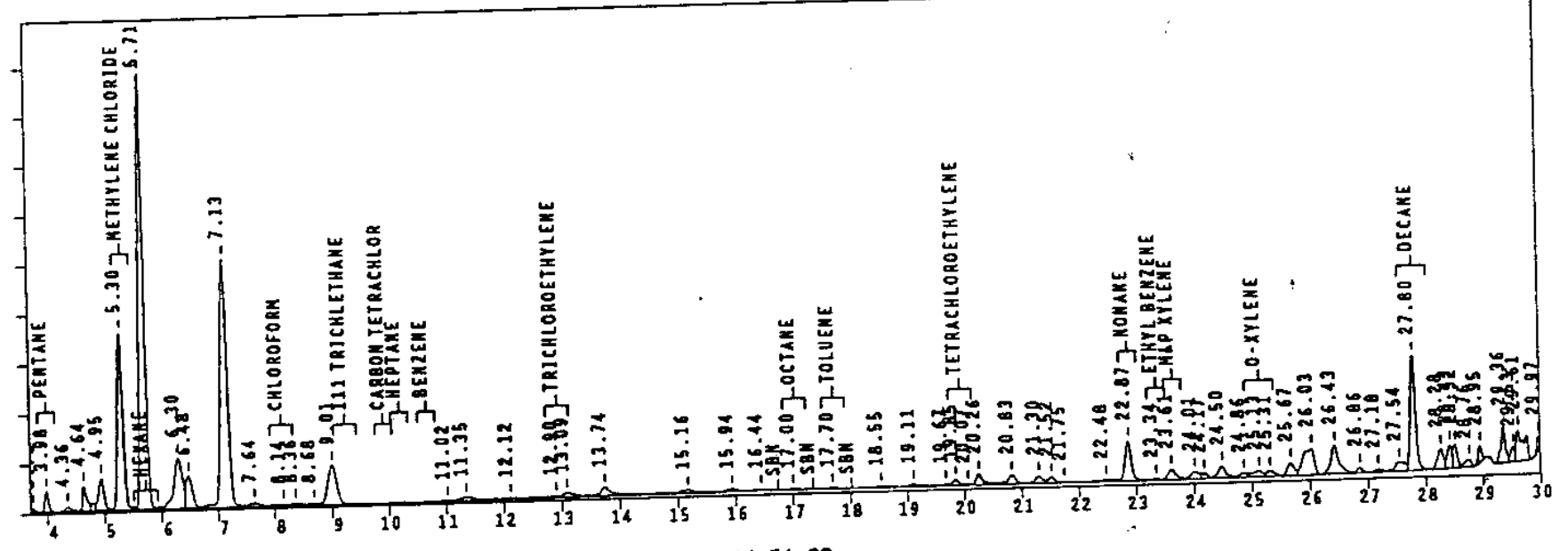

File $=C: \backslash C P \backslash d S \backslash W S A .36 R$ Date printed $=03-27-1991$ Time $=16: 54: 22$

3.70 to $29.92 \mathrm{~min}$. Lон $Y=-0.05649 \mathrm{mN}$ High $Y=0.47969 \mathrm{mV}$ Span $=0.53618 \mathrm{mV}$

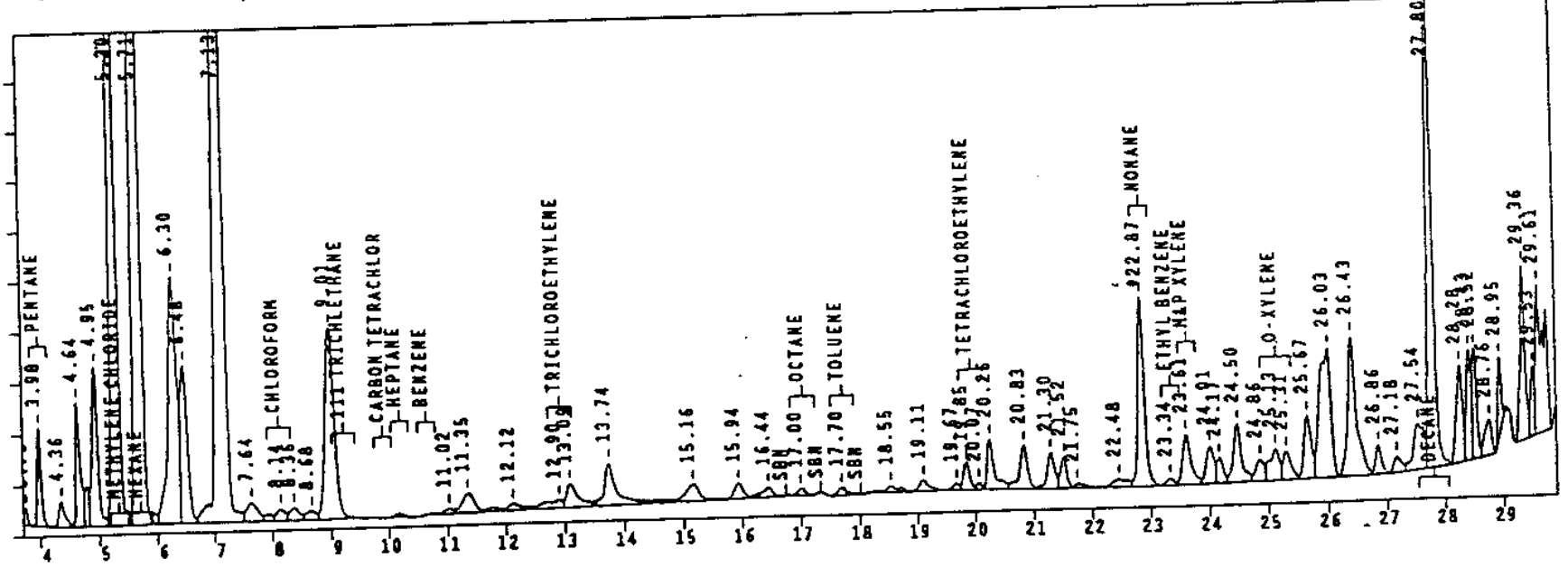

Figure 25. VoC's Under Groundsheet \#2 During a Period of Falling Barometric Pressure. (Ordinate scale successively expanded to reveal complex component distribution.) 


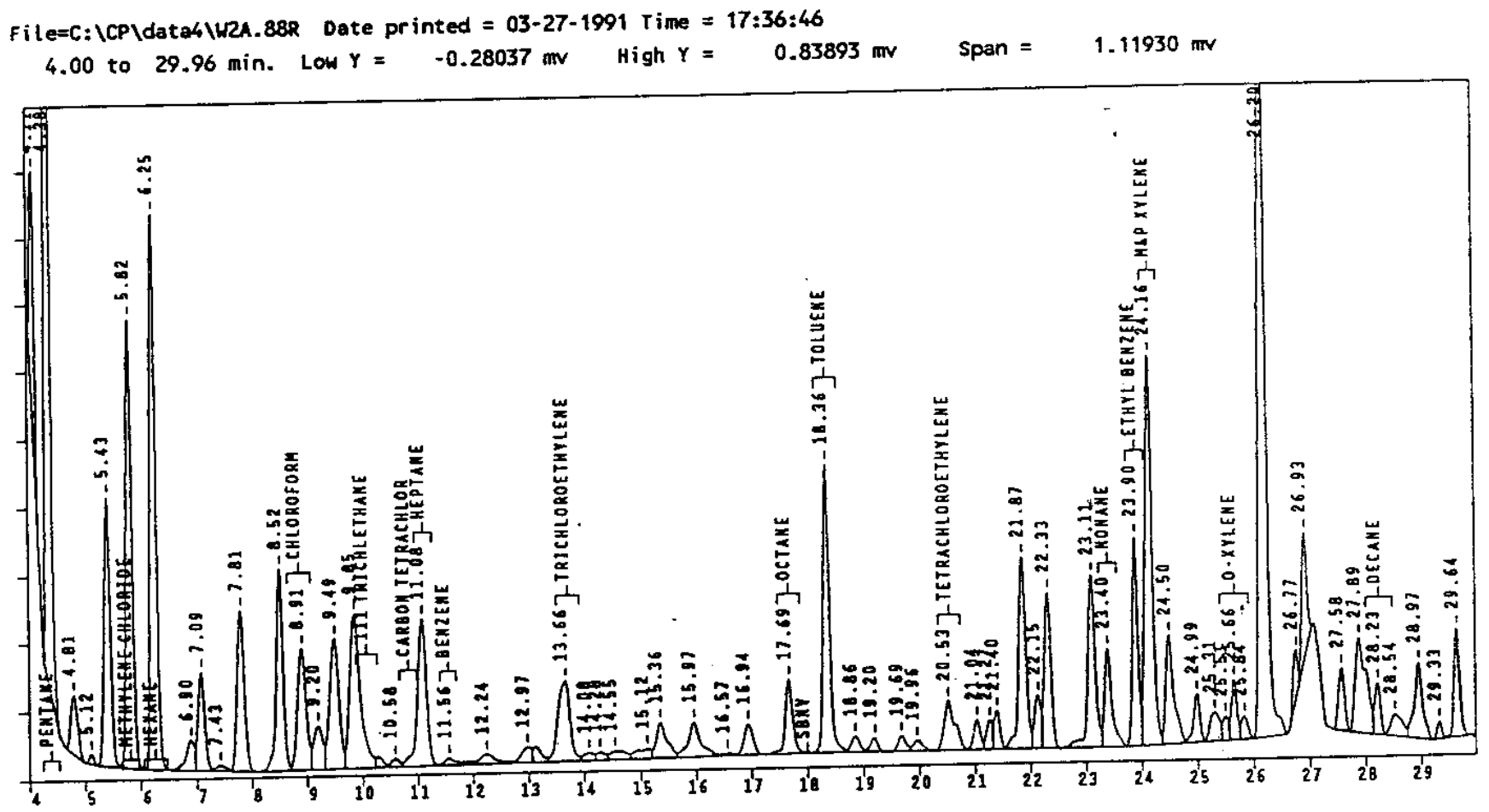
Figure 26. Voc's in the Burial Trench Beneath Groundsheet \#2 from
Soil Gas Site Location \#52 
Table 1 .

.. WESTINGHOUSE SAVANKAH RIVER SITE -..

.... LANDFILL GROUNDSHEET STUDY -..

.... SOIL GAS CONCENTRATIONS (PPMV)

\begin{tabular}{|c|c|c|c|c|c|c|c|c|c|c|}
\hline $\begin{array}{r}\text { SAMPLE } \\
\text { NAME }\end{array}$ & \multicolumn{4}{|c|}{ CYCLE } & \multirow{2}{*}{ ETHANE } & \multirow{2}{*}{ ETHYLENE } & \multicolumn{2}{|c|}{ PROPANE PROPYLENE } & \multirow[t]{2}{*}{ ISO-BUTANE } & \multirow{2}{*}{$\begin{array}{c}\text { N-BUTANE } \\
\text { - }\end{array}$} \\
\hline NAME & OATE & TIME & $\#$ & METHANE & & & & & & \\
\hline GS-1 & $9-5$ & 1002 & 5 & 2.053 & 0.032 & 0.072 & 0.917 & $<.005$ & 0.083 & 0.422 \\
\hline GS-1 & $1-5$ & 1358 & 9 & 2.431 & 0.056 & 0.095 & 0.122 & $<.005$ & $<.005$ & $<.005$ \\
\hline GS-1 & $1-5$ & 1745 & 13 & 2.363 & 0.039 & 0.077 & 0.079 & $<.005$ & $<.005$ & $<.005$ \\
\hline GS-1 & $1-5$ & 2220 & 20 & 2.252 & 0.048 & 0.089 & 0.045 & $<.005$ & $<.005$ & $<.005$ \\
\hline GS-1 & $1+6$ & 155 & 49 & 5.630 & 0.056 & 0.031 & 0.057 & $<.005$ & $<.005$ & $<.005$ \\
\hline GS-1 & 1.6 & 605 & 31 & 5.554 & 0.016 & $<.005$ & 0.074 & 0.026 & $<.005$ & $<.005$ \\
\hline GS-1 & $1-6$ & 1005 & 35 & 3.198 & 0.024 & 0.034 & 0.123 & $<.005$ & $<.005$ & 0.016 \\
\hline GS-1 & 1.6 & 1350 & 39 & 3.632 & 0.020 & 0.035 & 0.146 & $<.005$ & $<.005$ & $<.005$ \\
\hline GS-1 & $1-6$ & 1800 & 46 & 5.452 & 0.022 & 0.030 & 0.136 & $<.005$ & $<.005$ & $<.005$ \\
\hline GS. 1 & $1-6$ & 2200 & 51 & 4.455 & 0.019 & 0.022 & 0.099 & $<.005$ & $<.005$ & $<.005$ \\
\hline GS-1 & $1-7$ & 135 & 58 & 11.722 & 0.016 & 0.015 & 0.083 & 0.011 & $<.005$ & $<.005$ \\
\hline GS-1 & $1-7$ & 605 & 62 & 19.927 & 0.015 & 0.018 & 0.086 & $<.005$ & $<.005$ & $<.005$ \\
\hline GS-1 & 1.7 & 1005 & 66 & 9.479 & 0.018 & 0.013 & 0.085 & $<.005$ & $<.005$ & $<.005$ \\
\hline GS-1 & $1-7$ & 1355 & 74 & 3.850 & 0.018 & 0.022 & 0.118 & $<.005$ & $<.005$ & $<.005$ \\
\hline GS-1 & $1-7$ & 1755 & 78 & 3.328 & 0.023 & 0.044 & 0.219 & 3.810 & $<.005$ & $<.005$ \\
\hline GS-1 & 1.7 & 2150 & 85 & 2.514 & 0.018 & 0.018 & 0.159 & $<.005$ & $<.005$ & $<.005$ \\
\hline GS-1 & $1-8$ & 137 & 89 & 2.243 & 0.011 & 0.007 & 0.064 & $<.005$ & $<.005$ & $<.005$ \\
\hline GS-1 & $1-8$ & 602 & 93 & 2.151 & 0.016 & 0.013 & 0.059 & $<.005$ & $<.005$ & $<.005$ \\
\hline GS-1 & $1-8$ & 1005 & 100 & 2.111 & 0.020 & 0.021 & 0.052 & $<.005$ & $<.005$ & $<.005$ \\
\hline GS-1 & $1-8$ & 1405 & 104 & 2.226 & 0.009 & $<.005$ & 0.067 & $<.005$ & $<.005$ & $<.005$ \\
\hline GS- 1 & $1-8$ & 1750 & 111 & 2.141 & 0.012 & 0.023 & 0.056 & 0.006 & $<.005$ & $<.005$ \\
\hline GS-1 & $9-8$ & 2150 & 115 & 2.076 & 0.016 & 0.011 & 0.060 & $<.005$ & $<.005$ & $<.005$ \\
\hline GS-1 & $1-9$ & 150 & 119 & 2.228 & 0.017 & $<.005$ & 0.065 & $<.005$ & $<.005$ & $<.005$ \\
\hline GS-1 & $1-9$ & 605 & 126 & 2.151 & 0.011 & $<.005$ & 0.047 & 0.016 & $<.005$ & $<.005$ \\
\hline GS-1 & $1-9$ & 955 & 130 & 2.084 & 0.012 & 0.008 & 0.084 & $<.005$ & $<.005$ & $<.005$ \\
\hline GS-1 & $1-9$ & 1355 & 137 & 2.298 & 0.019 & $<.005$ & 0.109 & $<.005$ & $<.005$ & 0.018 \\
\hline GS-1 & $1-9$ & 1755 & 141 & 2.253 & 0.014 & 0.007 & 0.140 & 0.007 & $<.005$ & $<.005$ \\
\hline GS-1 & $1-9$ & 2150 & 145 & 2.051 & 0.015 & 0.005 & 0.090 & 0.020 & 0.006 & $<.005$ \\
\hline GS- 1 & $1-10$ & 142 & 152 & 2.407 & 0.011 & $<.005$ & 0.091 & $<.005$ & $<.005$ & $<.005$ \\
\hline GS- 1 & $1-10$ & 600 & 156 & 2.122 & 0.009 & $<.005$ & 0.078 & $<.005$ & $<.005$ & $<.005$ \\
\hline GS-1 & $1-10$ & 1005 & 164 & 2.032 & 0.012 & $<.005$ & 0.048 & $<.005$ & $<.005$ & $<.005$ \\
\hline GS-1 & $1-10$ & 1355 & 168 & 2.781 & 0.013 & $<.005$ & 0.129 & $<.005$ & $<.005$ & $<.005$ \\
\hline GS-1 & $1-10$ & 1755 & 172 & 2.539 & 0.009 & $<.005$ & 0.084 & $<.005$ & $<.005$ & $<.005$ \\
\hline GS-1 & $1-10$ & 1015 & 179 & 2.640 & 0.014 & $<.005$ & 0.046 & $<.005$ & $<.005$ & $<.005$ \\
\hline GS-1 & $1-11$ & 102 & 183 & 3.527 & 0.011 & $<.005$ & 0.052 & $<.005$ & $<.005$ & $<.005$ \\
\hline GS. 1 & $1-11$ & 600 & 190 & 4.221 & 0.022 & 0.027 & 0.186 & $<.005$ & $<.005$ & $<.005$ \\
\hline GS-1 & $1-11$ & 1001 & 194 & 3.672 & 0.039 & 0.025 & 0.346 & 0.038 & $<.005$ & $<.005$ \\
\hline GS-1 & $1-11$ & 1402 & 198 & 3.453 & 0.050 & 0.035 & 0.481 & 0.081 & $<.005$ & 0.103 \\
\hline GS-1 & $9-11$ & 1820 & 205 & 2.849 & 0.174 & 0.075 & 0.460 & $<.005$ & $<.005$ & $<.005$ \\
\hline GS-1 & $1-11$ & 2210 & 378 & 2.911 & 0.044 & 0.038 & 0.303 & 0.010 & $<.005$ & 0.006 \\
\hline GS-1 & $1-12$ & 135 & 216 & 2.625 & 0.038 & 0.037 & 0.401 & 0.064 & $<.005$ & $<.005$ \\
\hline GS- 1 & $1-12$ & 603 & 220 & 2.887 & 0.040 & 0.036 & 0.455 & 0.050 & $<.005$ & 0.053 \\
\hline GS-1 & $1-12$ & 958 & 224 & 2.797 & 0.037 & 0.030 & 0.479 & 0.086 & $<.005$ & 0.117 \\
\hline GS-1 & 1.12 & 1358 & 231 & 2.258 & 0.020 & 0.019 & 0.143 & 0.047 & $<.005$ & $<.005$ \\
\hline GS-1 & $1-12$ & 1755 & 235 & 2.040 & 0.016 & 0.017 & 0.086 & $<.005$ & $<.005$ & $<.005$ \\
\hline GS-1 & $1-12$ & 2200 & 242 & 1.982 & 0.011 & 0.012 & 0.082 & $<.005$ & $<.005$ & $<.005$ \\
\hline GS-1 & $1-13$ & 125 & 246 & 2.010 & 0.009 & 0.013 & 0.065 & $<.005$ & $<.005$ & $<.005$ \\
\hline GS-1 & $1-13$ & 608 & 250 & 2.049 & $<.005$ & 0.006 & 0.058 & $<.005$ & $<.005$ & 0.009 \\
\hline GS-1 & 1.13 & 1004 & 257 & 2.056 & 0.010 & 0.013 & 0.060 & $<.005$ & $<.005$ & $<.005$ \\
\hline GS-1 & $1-13$ & 1407 & 262 & 2.162 & 0.005 & 0.010 & 0.093 & $<.005$ & $<.005$ & $<.005$ \\
\hline GS- 1 & $1-13$ & 1753 & 269 & 2.050 & 0.015 & 0.016 & 0.062 & $<.005$ & $<.005$ & $<.005$ \\
\hline GS-1 & $1-13$ & 2240 & 273 & 2.322 & 0.025 & $<.005$ & 0.038 & $<.005$ & $<.005$ & $<.005$ \\
\hline & & 147 & 277 & 2.048 & 0.026 & 0.013 & 0.048 & $<.005$ & $<.005$ & $<.005$ \\
\hline
\end{tabular}

Table 1. C1-C4 Hydrocarbon Concentrations (ppmv) and system Blanks in the 740-G Landfill Groundsheet Study 
WESTINGHOUSE SAVANNAH RIVER SITE -...

LANDFILL GROUNDSHEET STUDY -...

SOIL GAS CONCENTRATIONS (PPHV)

\begin{tabular}{|c|c|c|c|c|c|c|c|c|c|c|}
\hline \multirow{2}{*}{$\begin{array}{r}\text { AMPLE } \\
\text { NAME }\end{array}$} & \multirow[b]{2}{*}{ DATE } & \multicolumn{3}{|c|}{ CYCLE } & \multirow[b]{2}{*}{ ETHANE } & \multirow[b]{2}{*}{ ETHYLENE } & \multirow{2}{*}{\multicolumn{2}{|c|}{ PROPANE PROPYLENE }} & \multirow{2}{*}{ ISO-BUTANE } & \multirow{2}{*}{ N-BUTANE } \\
\hline & & TIME & $\#$ & METKANE & & & & & & \\
\hline GS-1 & 1.14 & 610 & 284 & 2.036 & 0.019 & $<.005$ & 0.046 & $<.005$ & 0.007 & $<.005$ \\
\hline GS-1 & $1-14$ & 1008 & 288 & 2.042 & 0.011 & $<.005$ & 0.050 & $<.005$ & $<.005$ & $<.005$ \\
\hline GS-1 & $1-14$ & 1410 & 295 & 2.683 & 0.014 & 0.013 & 0.056 & 0.015 & $<.005$ & $<.005$ \\
\hline GS-1 & $1-14$ & 1800 & 299 & 5.321 & 0.018 & 0.017 & 0.081 & $<.005$ & $<.005$ & $<.005$ \\
\hline GS-1 & $1-14$ & 2200 & 303 & 2.317 & 0.011 & 0.008 & 0.036 & $<.005$ & $<.005$ & $<.005$ \\
\hline GS-1 & $1-15$ & 155 & 310 & 2.262 & 0.009 & $<.005$ & 0.030 & 0.021 & $<.005$ & $<.005$ \\
\hline GS-1 & $1-15$ & 615 & 314 & 2.688 & 0.015 & 0.007 & 0.043 & 0.007 & $<.005$ & $<.005$ \\
\hline GS-1 & 1.15 & 1004 & 321 & 2.190 & 0.021 & 0.008 & 0.062 & 0.009 & $<.005$ & $<.005$ \\
\hline GS - 1 & $1-15$ & 1407 & 325 & 4.555 & $<.005$ & 0.012 & 0.087 & $<.005$ & $<.005$ & $<.005$ \\
\hline GS-1 & $1+15$ & 1801 & 329 & 5.315 & 0.012 & 0.016 & 0.051 & $<.005$ & $<, 005$ & $<.005$ \\
\hline GS-1 & $1-15$ & 2205 & 336 & 4.566 & $<.005$ & 0.016 & 0.155 & $<.005$ & $<.005$ & $<.005$ \\
\hline GS-1 & $1-16$ & 144 & 339 & 2.995 & 0.029 & 0.024 & 0.287 & 0.013 & $<.005$ & $<.005$ \\
\hline GS-1 & $1-16$ & 601 & 344 & 3.407 & 0.026 & 0.016 & 0.282 & $<.005$ & $<.005$ & $<.005$ \\
\hline GS-1 & $1-16$ & 1002 & 347 & 2.698 & 0.028 & 0.030 & 0.475 & 0.080 & $<.005$ & $<.005$ \\
\hline GS-1 & $1-16$ & 1402 & 350 & 2.073 & 0.012 & 0.023 & 0.128 & $<.005$ & $<.005$ & $<.005$ \\
\hline GS-1 & $1-16$ & 1750 & 357 & 2.397 & 0.009 & 0.029 & 0.146 & $<.005$ & $<.005$ & $<.005$ \\
\hline GS-1 & $1-16$ & 2155 & 361 & 2.040 & 0.021 & 0.008 & 0.076 & $<.005$ & $<.005$ & $<.005$ \\
\hline GS-1 & $1-17$ & 230 & 368 & 1.946 & 0.016 & 0.010 & 0.048 & $<.005$ & $<.005$ & $<.005$ \\
\hline GS-1 & 1.17 & 607 & 372 & 1.961 & 0.006 & 0.006 & 0.057 & $<.005$ & $<.005$ & $<.005$ \\
\hline GS-1 & $1-17$ & 1009 & 379 & 1.854 & $<.005$ & 0.007 & 0.028 & $<.005$ & 0.006 & $<.005$ \\
\hline GS-1 & $1-17$ & 1418 & 386 & 2.029 & $<.005$ & $<.005$ & 0.057 & $<.005$ & $<.005$ & $<.005$ \\
\hline GS-1 & $1-17$ & 1759 & 390 & 1.928 & $<.005$ & 0.018 & 0.053 & $<.005$ & $<.005$ & $<.005$ \\
\hline GS-1 & $1-17$ & 2200 & 397 & 1.947 & 0.019 & $<.005$ & 0.060 & $<.005$ & $<.005$ & $<.005$ \\
\hline GS-1 & $1-18$ & 137 & 401 & 2.241 & 0.011 & 0.007 & 0.046 & $<.005$ & $<.005$ & $<.005$ \\
\hline GS-1 & $1-18$ & 610 & 405 & 2.045 & $<.005$ & $<.005$ & 0.037 & $<.005$ & $<.005$ & $<.005$ \\
\hline GS-1 & $1-18$ & 1004. & 412 & 1.946 & $<.005$ & 0.007 & 0.038 & $<.005$ & $<.005$ & $<.005$ \\
\hline GS-1 & $1-18$ & 1357 & 416 & 2.151 & $<.005$ & 0.050 & 0.054 & $<.005$ & $<.005$ & $<.005$ \\
\hline GS-1 & $1-18$ & 1807 & 424 & 1.957 & $<.005$ & 0.019 & 0.058 & $<.005$ & $<.005$ & $<.005$ \\
\hline GS- 1 & $1-18$ & 2226 & 428 & 3.652 & $<.005$ & $<.005$ & 0.041 & $<.005$ & $<.005$ & $<.005$ \\
\hline GS-1 & $1-19$ & 147 & 432 & 2.659 & $<.005$ & 0.009 & 0.037 & $<.005$ & $<.005$ & $<.005$ \\
\hline GS-1 & $1-19$ & 650 & 439 & 2.512 & 0.017 & 0.011 & 0.071 & $<.005$ & $<.005$ & $<.005$ \\
\hline GS-1 & $3-19$ & 1030 & 443 & 2.548 & 0.011 & 0.008 & 0.138 & $<.005$ & $<.005$ & 0.007 \\
\hline GS.2 & $1-5$ & 1012 & 6 & 24.517 & 0.038 & $<.005$ & 0.475 & 0.029 & 0.117 & 0.254 \\
\hline GS - 2 & $1-5$ & 1405 & 10 & 2447.979 & 0.077 & 0.022 & 0.701 & 0.138 & 0.949 & 0.203 \\
\hline GS-2 & 1.5 & 1750 & 14 & 1678.545 & 0.063 & $<.005$ & 0.356 & 0.109 & 0.616 & 0.125 \\
\hline GS-2 & $1-5$ & 2225 & 21 & 141.368 & 0.050 & 0.012 & 0.127 & 0.038 & 0.254 & $<.005$ \\
\hline GS-2 & $1-6$ & 200 & 25 & 311.009 & 0.023 & $<.005$ & 0.153 & 0.049 & 0.197 & $<.005$ \\
\hline GS-2 & 1.6 & 610 & 32 & 914.334 & 0.032 & $<.005$ & 0.329 & 0.085 & 0.490 & $<.005$ \\
\hline GS-2 & $1-6$ & 1010 & 36 & 347.830 & 0.023 & $<.005$ & 0.317 & 0.093 & 0.471 & $<.005$ \\
\hline GS - 2 & $1-6$ & 1355 & 40 & 8121.346 & 0.179 & 0.028 & 1.762 & 0.282 & 2.724 & 0.474 \\
\hline GS-2 & $1-6$ & 1805 & 47 & 7200.795 & 0.221 & 0.016 & 1.948 & 0.331 & 3.231 & 0.472 \\
\hline GS-2 & $1-6$ & 2205 & 52 & 1747.099 & 0.098 & 0.007 & 0.940 & 0.237 & 1.529 & $<.005$ \\
\hline GS-2 & $1-7$ & 140 & 59 & 1805.625 & 0.063 & 0.006 & 0.656 & 0.149 & 1.092 & $<.005$ \\
\hline GS-2 & $1-7$ & 610 & 63 & 10887.042 & 0.309 & 0.072 & 2.717 & 0.484 & 4.747 & 0.693 \\
\hline GS-2 & $1-7$ & 1010 & 67 & 6887.654 & 0.249 & 0.054 & 2.205 & 0.366 & 4.083 & 0.592 \\
\hline GS -2 & $1-7$ & 1400 & 75 & 6372.776 & 0.183 & 0.023 & 1.592 & 0.286 & 2.831 & 0.347 \\
\hline GS-2 & $1-7$ & 1800 & 79 & 8287.945 & 0.223 & 0.015 & 1.841 & 0.291 & 2.672 & $<.005$ \\
\hline GS-2 & $1-7$ & 2155 & 86 & 2470.374 & 0.080 & $<.005$ & 0.562 & 0.115 & 0.820 & $<.005$ \\
\hline GS-2 & $1-8$ & 141 & 90 & 499.245 & 0.028 & $<.005$ & 0.209 & $<.005$ & 0.275 & $-<.005$ \\
\hline GS -2 & 1.8 & 606 & 94 & 79.923 & 0.013 & $<.005$ & 0.105 & 0.021 & 0.058 & $<.005$ \\
\hline GS-2 & $1-8$ & 1010 & 101 & 16.882 & 0.011 & 0.008 & 0.054 & $<.005$ & $<.005$ & $<.005$ \\
\hline GS -2 & $1-8$ & 1410 & 105 & 1177.741 & 0.030 & $<.005$ & 0.251 & 0.036 & 0.257 & 0.005 \\
\hline
\end{tabular}


Table 1. (continued)

.. WESTINGHOUSE SAVANNAM RIVER SITE ....

.... LANDFILL GROUNDSHEET STUDY -..

.... SOIL GAS CONCENTRATIONS (PPMV)

\begin{tabular}{|c|c|c|c|c|c|c|c|c|c|c|}
\hline \multirow{2}{*}{$\begin{array}{r}\text { SAMPLE } \\
\text { NAME }\end{array}$} & \multicolumn{4}{|c|}{ CYCLE } & \multirow[b]{2}{*}{ ETHANE } & \multirow[b]{2}{*}{ ETHYLENE } & \multirow{2}{*}{\multicolumn{2}{|c|}{ PROPANE PROPYLENE }} & \multirow{2}{*}{ ISO-BUTANE } & \\
\hline & OATE & TIME & $\#$ & METHANE & & & & & & - \\
\hline GS - 2 & $1-8$ & 1755 & 112 & 469.964 & 0.011 & $<.005$ & 0.177 & $<.005$ & 0.150 & $<.005$ \\
\hline GS-2 & $1-8$ & 2155 & 116 & 20.805 & 0.013 & $<.005$ & 0.057 & $<.005$ & $<.005$ & $<.005$ \\
\hline GS-2 & $1-9$ & 154 & 120 & 10.218 & 0.026 & $<.005$ & 0.063 & $<.005$ & $<.005$ & $<.005$ \\
\hline$G S-2$ & $1-9$ & 610 & 127 & 6.039 & 0.012 & $<.005$ & 0.054 & $<.005$ & $<.005$ & $<.005$ \\
\hline GS-2 & $1-9$ & 1000 & 131 & 2.256 & 0.016 & $<.005$ & 0.054 & $<.005$ & $<.005$ & 0.005 \\
\hline GS-2 & $1-9$ & 1400 & 138 & 3152.993 & 0.094 & $<.005$ & 0.834 & $0.145 \cdots$ & 1.204 & $<.005$ \\
\hline GS-2 & $1-9$ & 1800 & 142 & 815.902 & 0.009 & $<.005$ & 0.336 & $<.005$ & 0.404 & $<.005$ \\
\hline GS-2 & $1-9$ & 2155 & 146 & 191.054 & 0.016 & $<.005$ & 0.223 & 0.038 & 0.169 & $<.005$ \\
\hline GS - 2 & $1-10$ & 146 & 153 & 72.897 & 0.017 & $<.005$ & 0.154 & 0.007 & 0.056 & $<.005$ \\
\hline GS-2 & $1-10$ & 604 & 157 & 14.689 & 0.014 & $<.005$ & 0.066 & $<.005$ & $<.005$ & $<.005$ \\
\hline GS-2 & $1-10$ & 1008 & 165 & 3.505 & 0.007 & $<.005$ & 0.050 & $<.005$ & $<.005$ & $<.005$ \\
\hline GS-2 & $1-10$ & 1400 & 169 & 4614.336 & 0.104 & 0.011 & 0.879 & 0.128 & 1.246 & $<.005$ \\
\hline GS-2 & $1-10$ & 1800 & 173 & 1814.039 & $<.005$ & $<.005$ & 0.364 & 0.051 & 0.460 & $<.005$ \\
\hline GS-2 & $1-10$ & 1020 & 180 & 922.349 & 0.027 & $<.005$ & 0.191 & $<.005$ & 0.177 & 0.005 \\
\hline GS-2 & $1 \cdot 11$ & 105 & 184 & 5300.271 & 0.122 & 0.014 & 1.057 & 0.019 & 1.689 & 0.288 \\
\hline GS - 2 & $1-11$ & 604 & 191 & 11951.161 & 0.109 & 0.030 & 2.240 & $<.005$ & 3.448 & 0.496 \\
\hline GS-2 & $1-11$ & 1004 & 195 & 15119.392 & 0.163 & 0.045 & 3.313 & 0.433 & 5.244 & 0.851 \\
\hline GS-2 & $1-11$ & 1406 & 199 & 14711.763 & 0.146 & 0.042 & 2.986 & $<.005$ & 4.597 & 0.816 \\
\hline GS - 2 & $1-11$ & 1825 & 206 & 2129.815 & 0.060 & 0.023 & 0.518 & $<.005$ & 0.567 & $<.005$ \\
\hline GS - 2 & $1-11$ & 2215 & 210 & 5694.337 & 0.125 & $<.005$ & 1.051 & 0.122 & 1.366 & 0.279 \\
\hline GS-2 & $1-12$ & 140 & 217 & 12754.938 & 0.270 & $<.005$ & 2.170 & 0.274 & 3.184 & 0.645 \\
\hline $\mathrm{GS}-2$ & $1-12$ & 606 & 221 & 20522.000 & 0.501 & 0.093 & 4.189 & 0.519 & 6.367 & 1.208 \\
\hline GS-2 & $1-12$ & 1001 & 225 & 20324.857 & 0.499 & 0.078 & 4.015 & 0.499 & 6.125 & 1.237 \\
\hline GS-2 & $1-12$ & 1401 & 232 & 6820.810 & 0.157 & 0.040 & 1.406 & 0.125 & 2.033 & 0.422 \\
\hline GS-2 & $1-12$ & 1758 & 236 & 503.894 & 0.021 & $<.005$ & 0.130 & $<.005$ & 0.096 & $<.005$ \\
\hline$G S-2$ & $1-12$ & 2205 & 243 & 73.637 & 0.016 & $<.005$ & 0.052 & $<.005$ & $<.005$ & $<.005$ \\
\hline GS-2 & $1-13$ & 129 & 247 & 67.313 & $<.005$ & $<.005$ & 0.049 & $<.005$ & $<.005$ & $<.005$ \\
\hline GS-2 & $1-13$ & 611 & 251 & 38.096 & 0.018 & 0.008 & 0.056 & $<.005$ & $<.005$ & $<.005$ \\
\hline GS-2 & $1-13$ & 1007 & 259 & 6.480 & 0.009 & $<.005$ & 0.010 & $<.005$ & $<.005$ & $<.005$ \\
\hline$G S-2$ & $1-13$ & 1410 & 263 & 882.031 & 0.029 & $<.005$ & 0.194 & $<.005$ & 0.174 & $<.005$ \\
\hline GS-2 & $1-13$ & 1757 & 270 & 256.149 & 0.017 & 0.013 & 0.130 & $<.005$ & 0.008 & $<.005$ \\
\hline GS-2 & $1-13$ & 2250 & 274 & 21.631 & 0.018 & $<.005$ & 0.055 & $<.005$ & $<.005$ & $<.005$ \\
\hline GS-2 & $1-14$ & 152 & 278 & 13.770 & 0.008 & $<.005$ & 0.025 & $<.005$ & $<.005$ & 0.006 \\
\hline GS-2 & $1-14$ & 613 & 285 & 8.265 & 0.012 & $<.005$ & 0.031 & $<.005$ & $<.005$ & $<.005$ \\
\hline GS-2 & $1-14$ & 1011 & 289 & 4.319 & 0.015 & $<.005$ & 0.025 & 0.008 & $<.005$ & $<.005$ \\
\hline GS-2 & $1 \cdot 14$ & 1415 & 296 & 2010.711 & 0.055 & 0.016 & 0.386 & 0.069 & 0.434 & $<.005$ \\
\hline GS-2 & $1-14$ & 1804 & 300 & 1420.335 & 0.027 & $<.005$ & 0.331 & 0.619 & 0.414 & $<.005$ \\
\hline GS-2 & $1-14$ & 2205 & 304 & 341.238 & 0.005 & $<.005$ & 0.217 & 0.055 & 0.285 & $<.005$ \\
\hline GS-2 & $1-15$ & 159 & 311 & 94.182 & 0.008 & $<.005$ & 0.113 & $<.005$ & 0.149 & $<.005$ \\
\hline GS-2 & $1-15$ & 618 & 315 & 2464.269 & 0.074 & $<.005$ & 0.590 & 0.105 & 0.926 & 0.137 \\
\hline GS-2 & $1-15$ & 1007 & 322 & 452.955 & $<.005$ & $<.005$ & 0.122 & $<.005$ & 0.145 & $<.005$ \\
\hline GS - 2 & $1-15$ & 1411 & 326 & 33.514 & 0.005 & 0.011 & 0.016 & $<.005$ & $<.005$ & $<.005$ \\
\hline GS-2 & $1-15$ & 1804 & 331 & 60.816 & $<.005$ & $<.005$ & 0.014 & $<.005$ & $<.005$ & $<.005$ \\
\hline GS-2 & $1-15$ & 2210 & 337 & 5744.961 & 0.156 & 0.056 & 1.364 & 0.183 & 2.216 & 0.460 \\
\hline GS-2 & $1+16$ & 149 & 340 & 8494.188 & 0.131 & 0.029 & 1.640 & 0.219 & 2.691 & 0.517 \\
\hline GS-2 & $1-16$ & 605 & 345 & 11520.146 & 0.238 & 0.031 & 2.259 & 0.277 & 3.526 & 0.685 \\
\hline $\mathrm{GS} \cdot 2$ & $1-96$ & 1006 & 348 & 5110.497 & 0.119 & 0.017 & 1.183 & 0.175 & 1.675 & 0.332 \\
\hline GS-2 & $1-16$ & 1406 & 351 & 658.530 & 0.014 & 0.017 & 0.149 & 0.023 & 0.145 & $<.005$ \\
\hline GS-2 & $1-16$ & 1755 & 358 & 421.314 & 0.009 & $<.005$ & 0.122 & $<.005$ & 0.066 & $-<.005$ \\
\hline GS-2 & $9-16$ & 2200 & 362 & 23.322 & 0.009 & 0.008 & 0.033 & $<.005$ & $<.005$ & $<.005$ \\
\hline GS-2 & $1 \cdot 17$ & 234 & 369 & 5.934 & $<.005$ & 0.008 & 0.025 & $<.005$ & 0.007 & $<.005$ \\
\hline$G S-2$ & $1-17$ & 609 & 373 & 5.232 & 0.005 & $<.005$ & 0.037 & $<.005$ & $<.005$ & $<.005$ \\
\hline & $1-17$ & 1012 & 380 & 2.947 & $<.005$ & $<.005$ & 0.035 & 0.016 & $<.005$ & $<.005$ \\
\hline
\end{tabular}


Table 1. (continued)

- WESTINGHOUSE SAVANHAH RIVER SITE

.... LANDFILL GROUNDSHEET STUDY -...

... SOIL GAS CONCENTRATIONS (PPMV)

\begin{tabular}{|c|c|c|c|c|c|c|c|c|c|c|}
\hline SAMPLE & & & YYCLE & & & & & & ISO-BUTANE & N-BUTANE \\
\hline NAME & $\begin{array}{l}\text { DATE } \\
\ldots . . .\end{array}$ & TIME & $\#$ & METKANE & ETHANE & ETHYLENE & PROPANE & PROPYLEAE & - & $\cdots+$ \\
\hline GS-2 & $1-17$ & 1422 & 387 & 545.108 & $<.005$ & 0.006 & 0.093 & $<.005$ & 0.093 & $<.005$ \\
\hline GS-2 & $1-17$ & 1802 & 391 & 44.491 & 0.009 & 0.013 & 0.058 & $<.005$ & 0.028 & $<.005$ \\
\hline GS-2 & $1 \cdot 17$ & 2203 & 398 & 7.471 & 0.007 & $<.005$ & 0.033 & $<.005$ & $<.005$ & 0.005 \\
\hline GS -2 & $1-18$ & 141 & 402 & 11.808 & 0.007 & 0.007 & 0.029 & 0.031 & $<.005$ & $<.005$ \\
\hline GS-2 & $1-18$ & 614 & 406 & 20.975 & 0.011 & $<.005$ & 0.033 & $<.005$ & $<.005$ & $<.005$ \\
\hline GS-2 & $1-18$ & 1007 & 413 & 4.006 & $<.005$ & $<.005$ & 0.024 & $<.005$ & $<.005$ & $<.005$ \\
\hline$G S-2$ & $1-18$ & 1400 & 417 & 350.132 & 0.008 & $<.005$ & 0.074 & $<.005$ & 0.007 & $<.005$ \\
\hline GS-2 & $1-18$ & 1810 & 425 & 143.348 & 0.015 & 0.011 & 0.078 & $<.005$ & $<.005$ & $<.005$ \\
\hline GS-2 & $1+18$ & 2230 & 429 & 45.470 & 0.005 & $<.005$ & 0.073 & $<.005$ & $<.005$ & $<.005$ \\
\hline GS-2 & $1-19$ & 150 & 433 & 61.819 & $<.005$ & $<.005$ & 0.065 & 0.008 & 0.035 & $<.005$ \\
\hline GS-2 & $1-19$ & 654 & 440 & 1544.672 & 0.029 & $<.005$ & 0.267 & 0.037 & 0.341 & $<.005$ \\
\hline$G S-2$ & $1-19$ & 1036 & 444 & 2095.233 & 0.052 & $<.005$ & 0.492 & 0.060 & 0.706 & 0.076 \\
\hline GS -3 & $1-5$ & 1020 & 7 & 1.930 & 0.027 & 0.014 & 0.286 & $<.005$ & $<.005$ & 0.110 \\
\hline GS-3 & $1-5$ & 1490 & 11 & 2.048 & 0.058 & 0.107 & 0.090 & $<.005$ & $<.005$ & $<.005$ \\
\hline GS-3 & $1-5$ & 1800 & 18 & 2.012 & 0.052 & 0.059 & 0.055 & 0.036 & $<.005$ & $<.005$ \\
\hline GS -3 & $1-5$ & 2230 & 22 & 1.992 & 0.049 & 0.016 & 0.057 & 0.062 & $<.005$ & $<.005$ \\
\hline GS -3 & $1-6$ & 205 & 26 & 2.411 & 0.023 & 0.015 & 0.079 & $<.005$ & $<.005$ & $<.005$ \\
\hline GS -3 & $1-6$ & 615 & 33 & 2.629 & 0.011 & 0.010 & 0.090 & $<.005$ & $<.005$ & $<.005$ \\
\hline GS-3 & $1-6$ & 1015 & 37 & 2.256 & 0.023 & 0.015 & 0.127 & 0.029 & $<.005$ & 0.007 \\
\hline $\mathrm{GS}-3$ & $1-6$ & 1405 & 44 & 2.366 & 0.025 & 0.026 & 0.193 & 0.074 & $<.005$ & 0.040 \\
\hline GS-3 & $1-6$ & 1810 & 48 & 3.250 & 0.022 & 0.053 & 0.173 & 0.056 & $<.005$ & $<.005$ \\
\hline GS-3 & $1-6$ & 2210 & 53 & 3.145 & 0.007 & 0.012 & 0.124 & 0.029 & $<.005$ & $<.005$ \\
\hline GS-3 & $1-7$ & 145 & 60 & 5.059 & 0.018 & 0.019 & 0.117 & 0.039 & $<.005$ & $<.005$ \\
\hline GS -3 & $1-7$ & 615 & 64 & 7.559 & 0.014 & 0.017 & 0.104 & $<.005$ & 0.033 & $<.005$ \\
\hline GS-3 & $1-7$ & 1020 & 71 & 4.822 & 0.016 & 0.019 & 0.114 & $<.005$ & $<.005$ & $<.005$ \\
\hline GS -3 & $1-7$ & 1405 & 76 & 2.953 & 0.018 & 0.036 & 0.094 & $<.005$ & $<.005$ & $<.005$ \\
\hline GS-3 & $1-7$ & 1805 & 80 & 2.442 & 0.019 & 0.031 & 0.120 & 0.045 & $<.005$ & $<.005$ \\
\hline GS -3 & $1-7$ & 2200 & 87 & 2.328 & 0.022 & 0.021 & 0.103 & 0.010 & $<.005$ & $<.005$ \\
\hline GS -3 & $1-8$ & 145 & 91 & 2.550 & 0.019 & 0.016 & 0.062 & $<.005$ & $<.005$ & $<.005$ \\
\hline GS-3 & $1-8$ & 615 & 98 & 2.116 & 0.015 & 0.010 & 0.090 & $<.005$ & $<.005$ & $<.005$ \\
\hline GS-3 & $.1-8$ & 1015 & 102 & 2.121 & 0.011 & 0.010 & 0.089 & $<.005$ & $<.005$ & $<.005$ \\
\hline GS-3 & $1-8$ & 1414 & 106 & 2.220 & 0.010 & 0.010 & 0.087 & $<.005$ & $<.005$ & $<.005$ \\
\hline GS -3 & $1-8$ & 1800 & 113 & 2.202 & 0.015 & 0.035 & 0.101 & $<.005$ & $<.005$ & 0.006 \\
\hline GS - 3 & $1-8$ & 2200 & 117 & 2.077 & 0.012 & 0.009 & 0.075 & $<.005$ & $<.005$ & $<.005$ \\
\hline$G S-3$ & $9-9$ & 203 & 124 & 2.034 & 0.010 & $<.005$ & 0.072 & $<.005$ & $<.005$ & $<.005$ \\
\hline GS-3 & $1-9$ & 613 & 128 & 2.047 & 0.011 & 0.006 & 0.059 & $<.005$ & $<.005$ & $<.005$ \\
\hline GS-3 & $1-9$ & 1005 & 132 & 2.025 & 0.013 & 0.009 & 0.066 & $<.005$ & $<.005$ & $<.005$ \\
\hline GS -3 & $1-9$ & 1405 & 139 & 2.091 & 0.024 & $<.005$ & 0.071 & $<.005$ & $<.005$ & 0.017 \\
\hline GS -3 & $1-9$ & 1803 & 143 & 2.044 & 0.027 & $<.005$ & 0.072 & $<.005$ & $<.005$ & $<.005$ \\
\hline GS-3 & $1-9$ & 2205 & 150 & 2.042 & 0.012 & 0.011 & 0.101 & 0.017 & $<.005$ & $<.005$ \\
\hline GS - 3 & $1-10$ & 150 & 154 & 2.025 & $<.005$ & $<.005$ & 0.100 & $<\infty 005$ & $<.005$ & $<.005$ \\
\hline GS -3 & $1-10$ & 608 & 158 & 1.986 & 0.011 & $<.005$ & 0.069 & $<.005$ & $<.005$ & $<.005$ \\
\hline GS-3 & $1-10$ & 1011 & 166 & 1.995 & 0.014 & $<.005$ & 0.091 & $<.005$ & $<.005$ & $<.005$ \\
\hline GS -3 & $1-10$ & 1403 & 170 & 2.090 & 0.013 & $<.005$ & 0.077 & $<.005$ & 0.007 & 0.023 \\
\hline GS - 3 & $1-10$ & 1808 & 177 & 1.987 & 0.011 & 0.009 & 0.065 & $<.005$ & $<.005$ & $<.005$ \\
\hline GS -3 & $1-10$ & 1025 & 181 & 1.960 & 0.010 & $<.005$ & 0.036 & 0.010 & $<.005$ & $<.005$ \\
\hline GS -3 & $1-11$ & 109 & 185 & 2.116 & 0.012 & $<.005$ & 0.062 & $<.005$ & $<.005$ & $<.005$ \\
\hline GS-3 & $1-11$ & 607 & 192 & 2.637 & 0.027 & 0.015 & 0.218 & 0.009 & $<.005$ & $<.005$ \\
\hline GS -3 & $1-11$ & 1010 & 196 & 2.651 & 0.037 & 0.020 & 0.339 & 0.087 & $<.005$ & 0.032 \\
\hline GS -3 & $1-11$ & 1415 & 203 & 2.605 & 0.095 & 0.063 & 0.243 & 0.072 & $<.005$ & 0.013 \\
\hline GS -3 & $1-11$ & 1830 & 376 & 2.206 & 0.009 & 0.013 & 0.109 & $<.005$ & $<.005$ & $<.005$ \\
\hline GS-3 & $9-1\}$ & 2220 & 211 & 2.177 & 0.012 & 0.009 & 0.133 & 0.051 & $<.005$ & $<.005$ \\
\hline
\end{tabular}


Table 1. (continued)

.. WESTINGHOUSE SAVANMAK RIVER SITE -...

.... LANDFILL GROUNDSHEET STUDY -...

.... SOIL GAS CONCENTRATIONS (PPMV)

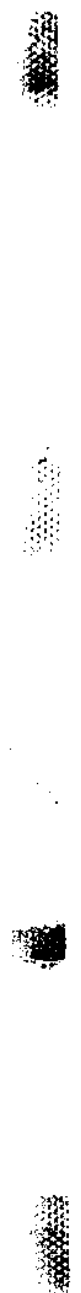

\begin{tabular}{|c|c|c|c|c|c|c|c|c|c|c|}
\hline \multirow{2}{*}{$\begin{array}{r}\text { SAMPLE } \\
\text { NAME }\end{array}$} & \multirow[b]{2}{*}{ DATE } & \multicolumn{3}{|c|}{ CYCLE } & \multirow[b]{2}{*}{ ETHANE } & \multirow[b]{2}{*}{ ETHYLENE } & \multirow{2}{*}{\multicolumn{2}{|c|}{ PROPANE PROPYLENE }} & \multirow{2}{*}{ ISO-BUTANE } & \multirow{2}{*}{ N-BUTANE } \\
\hline & & TIME & $\#$ & METHANE & & & & & & \\
\hline GS-3 & $1-12$ & 145 & 218 & 2.413 & 0.013 & 0.016 & 0.221 & $<.005$ & $<.005$ & $<.005$ \\
\hline GS-3 & $9-12$ & 610 & 222 & 2.713 & 0.025 & 0.007 & 0.346 & 0.083 & 0.008 & 0.055 \\
\hline GS -3 & $1-12$ & 1009 & 229 & 2.731 & 0.028 & 0.023 & 0.387 & $<.005$ & $<.005$ & 0.055 \\
\hline GS-3 & $1-12$ & 1405 & 233 & 2.407 & 0.018 & 0.006 & 0.060 & 0.008 & $<.005$ & $<.005$ \\
\hline GS -3 & $1-12$ & 1801 & 237 & 2.017 & 0.011 & $<.005$ & 0.024 & $<.005$ & $<.005$ & $<.005$ \\
\hline GS -3 & $1-12$ & 2210 & 244 & 1.976 & 0.012 & 0.005 & 0.027 & $<.005^{\prime \prime}$ & $<.005$ & $<.005$ \\
\hline GS -3 & $1-13$ & 133 & 248 & 2.002 & 0.027 & $<.005$ & 0.015 & $<.005$ & $<.005$ & 0.012 \\
\hline$G S-3$ & $1-13$ & 618 & 255 & 1.990 & 0.017 & 0.008 & 0.043 & $<.005$ & $<.005$ & $<.005$ \\
\hline GS -3 & $1-13$ & 1011 & 260 & 2.038 & 0.011 & 0.005 & 0.035 & $<.005$ & $<.005$ & $<.005$ \\
\hline GS-3 & $1-13$ & 1414 & 264 & 2.122 & 0.013 & 0.014 & 0.104 & $<.005$ & 0.005 & 0.005 \\
\hline GS-3 & $1-13$ & 1801 & 271 & 2.032 & 0.007 & $<.005$ & 0.029 & $<.005$ & $<.005$ & $<.005$ \\
\hline GS-3 & $1-13$ & 2303 & 275 & 2.200 & 0.979 & $<.005$ & 0.037 & $<.005$ & $<.005$ & 0.007 \\
\hline GS-3 & $1-14$ & 205 & 282 & 2.003 & $<.005$ & $<.005$ & 0.051 & $<.005$ & $<.005$ & 0.008 \\
\hline GS -3 & $1-14$ & 618 & 286 & 2.003 & 0.015 & $<.005$ & 0.057 & $<.005$ & $<.005$ & $<.005$ \\
\hline GS-3 & $1-14$ & 1013 & 290 & 2.046 & 0.011 & 0.006 & 0.057 & $<.005$ & $<.005$ & 0.035 \\
\hline GS-3 & $1 \cdot 14$ & 1420 & 297 & 2.000 & 0.026 & 0.021 & 0.113 & $<.005$ & $<.005$ & $<.005$ \\
\hline GS -3 & $1-14$ & 1809 & 301 & 2.964 & 0.014 & 0.017 & 0.064 & $<.005$ & $<.005$ & $<.005$ \\
\hline GS -3 & $1-14$ & 2214 & 308 & 2.003 & 0.005 & $<.005$ & 0.032 & 0.215 & $<.005$ & $<.005$ \\
\hline GS-3 & $1-15$ & 205 & 312 & 1.926 & 0.012 & $<.005$ & 0.041 & $<.005$ & $<.005$ & $<.005$ \\
\hline GS-3 & $1-15$ & 621 & 316 & 1.991 & $<.005$ & 0.009 & 0.048 & $<.005$ & $<.005$ & $<.005$ \\
\hline GS-3 & $1-15$ & 1010 & 323 & 1.962 & 0.016 & 0.008 & 0.042 & 0.005 & $<.005$ & $<.005$ \\
\hline GS-3 & $1-15$ & 1414 & 327 & 1.954 & 0.005 & 0.016 & 0.014 & $<.005$ & $<.005$ & $<.005$ \\
\hline GS -3 & $1-15$ & 1812 & 334 & 1.899 & $<.005$ & 0.023 & 0.024 & $<.005$ & $<.005$ & $<.005$ \\
\hline GS-3 & $9-15$ & 2215 & 338 & 2.043 & 0.007 & 0.020 & 0.118 & 0.031 & $<.005$ & $<.005$ \\
\hline GS-3 & $1-16$ & 154 & 341 & 2.920 & $<.005$ & 0.010 & 0.097 & $<.005$ & $<.005$ & 0.005 \\
\hline GS-3 & $1-16$ & 611 & 346 & 2.027 & $<.005$ & 0.013 & 0.069 & 0.009 & $<.005$ & $<.005$ \\
\hline GS -3 & $1-16$ & 1011 & 349 & 2.017 & 0.006 & 0.014 & 0.199 & 0.040 & $<.005$ & $<.005$ \\
\hline GS -3 & $1 \cdot 16$ & 1413 & 355 & 2.032 & $<.005$ & 0.020 & 0.024 & $<.005$ & $<.005$ & $<.005$ \\
\hline GS-3 & $1-16$ & 1800 & 359 & 1.897 & $<.005$ & 0.016 & 0.054 & 0.008 & $<.005$ & $<.005$ \\
\hline GS-3 & $1-16$ & 2203 & 363 & 1.976 & 0.010 & 0.011 & 0.050 & $<.005$ & $<.005$ & $<.005$ \\
\hline GS - 3 & $1-17$ & 238 & 370 & 1.905 & $<.005$ & 0.009 & 0.024 & 0.009 & $<.005$ & $<.005$ \\
\hline GS-3 & $9-17$ & 613 & 374 & 1.915 & 0.009 & 0.006 & 0.030 & $<.005$ & $<.005$ & $<.005$ \\
\hline GS-3 & $1-17$ & 1018 & 384 & 1.888 & $<.005$ & 0.015 & 0.020 & $<.005$ & $<.005$ & $<.005$ \\
\hline GS -3 & $1-17$ & 1426 & 388 & 1.928 & 0.023 & 0.014 & 0.053 & $<.005$ & $<.005$ & $<.005$ \\
\hline GS-3 & $1-17$ & 1806 & 392 & 1.901 & 0.023 & 0.017 & 0.045 & $<.005$ & $<.005$ & $<.005$ \\
\hline GS-3 & $1 \cdot 17$ & 2206 & 399 & 1.883 & 0.016 & 0.008 & 0.051 & $<.005$ & $<.005$ & $<.005$ \\
\hline GS-3 & $1-18$ & 145 & 403 & 1.996 & 0.019 & 0.007 & 0.051 & 0.005 & $<.005$ & $<.005$ \\
\hline GS-3 & $1-18$ & 621 & 410 & 1.908 & $<.005$ & $<.005$ & 0.037 & $<.005$ & $<.005$ & $<.005$ \\
\hline GS-3 & $1-18$ & 1011 & 414 & 1.923 & $<.005$ & 0.007 & 0.051 & $<.005$ & $<.005$ & $<.005$ \\
\hline GS-3 & $1-18$ & 1403 & 418 & 1.903 & $<.005$ & $<.005$ & 0.026 & $<.005$ & $<.005$ & $<.005$ \\
\hline GS-3 & $1-18$ & 1813 & 426 & 1.927 & 0.013 & 0.017 & 0.039 & $<.005$ & $<.005$ & $<.005$ \\
\hline GS-3 & $1-18$ & 2234 & 430 & 2.183 & $<.005$ & 0.007 & 0.039 & $<.005$ & $<.005$ & $<.005$ \\
\hline GS-3 & $1-19$ & 158 & 437 & 1.946 & $<.005$ & 0.010 & 0.053 & $<.005$ & 0.009 & $<.005$ \\
\hline$G S-3$ & $1-19$ & 658 & 441 & 1.955 & $<.005$ & 0.007 & 0.089 & $<.005$ & $<.005$ & $<.005$ \\
\hline$G S+3$ & $1-19$ & 1040 & 445 & 1.931 & $<.005$ & 0.010 & 0.061 & $<.005$ & $<.005$ & $<.005$ \\
\hline GS-4 & $1-5$ & 1028 & 8 & 1.963 & 0.063 & $<.005$ & 0.383 & 0.048 & $<.005$ & 0.202 \\
\hline GS-4 & $1-5$ & 1415 & 12 & 2.044 & 0.056 & 0.022 & 0.119 & 0.053 & $<.005$ & $<.005$ \\
\hline GS-4 & 1.5 & 1810 & 19 & 2.017 & 0.052 & 0.112 & 0.085 & 0.060 & $<.005$ & $<.005$ \\
\hline GS-4 & $1-5$ & 2235 & 23 & 1.988 & 0.050 & $<.005$ & 0.072 & $<.005$ & $<.005$ & $<.005$ \\
\hline GS-4 & $1-6$ & 210 & 27 & 2.113 & 0.022 & 0.012 & 0.078 & $<.005$ & $<.005$ & $<.005$ \\
\hline GS- 4 & 1.6 & 620 & 34 & 2.350 & 0.019 & 0.016 & 0.092 & $<.005$ & $<.005$ & $<.005$ \\
\hline & $1-6$ & 1020 & 38 & 2.191 & 0.021 & 0.020 & 0.135 & $<.005$ & $<.005$ & $<.005$ \\
\hline
\end{tabular}


Table 1. (continued)

.... MESTINGHOUSE SAVANMAH RIVER SITE -....

-.... LANDFILL GROUNDSHEET STUDY -..

..... SOIL GAS CONCENTRATIONS (PPMV)

\begin{tabular}{|c|c|c|c|c|c|c|c|c|c|c|}
\hline $\begin{array}{r}\text { SAMPLE } \\
\text { NAME }\end{array}$ & \multirow[b]{2}{*}{ DATE } & \multicolumn{3}{|c|}{ CYCLE } & \multirow[b]{2}{*}{ ETHANE } & \multirow{2}{*}{ ETHYLENE } & \multirow{2}{*}{\multicolumn{2}{|c|}{ PROPANE PROPYLENE }} & ISO-BUTANE & \multirow[t]{2}{*}{ N-BUTANE } \\
\hline $\begin{array}{c}\text { NAME } \\
.\end{array}$ & & TIME & $\#$ & METHANE & & & & & & \\
\hline GS -4 & $1-6$ & 1410 & 45 & 2.231 & 0.027 & 0.026 & 0.221 & 0.048 & $<.005$ & $<.005$ \\
\hline GS -4 & $1-6$ & 1815 & 50 & 2.359 & 0.032 & 0.066 & 0.297 & 0.088 & $<.005$ & $<.005$ \\
\hline GS-4 & $1-6$ & 2215 & 54 & 2.468 & 0.016 & 0.018 & 0.165 & 0.064 & $<.005$ & $<.005$ \\
\hline GS -4 & $1-7$ & 150 & 61 & 3.013 & 0.017 & 0.016 & 0.134 & 0.041 & $<.005$ & $<.005$ \\
\hline GS-4 & $1-7$ & 620 & 65 & 4.086 & 0.015 & 0.019 & 0.124 & $<.005$ & $<.005$ & $<.005$ \\
\hline GS -4 & $1-7$ & 1025 & 72 & 4.196 & 0.021 & 0.026 & 0.137 & $<.005$ & 0.016 & $<.005$ \\
\hline GS -4 & $1-7$ & 1410 & 77 & 3.058 & 0.022 & 0.035 & 0.121 & 0.057 & $<.005$ & $<.005$ \\
\hline GS -4 & $1+7$ & 1810 & 81 & 2.403 & 0.020 & 0.038 & 0.191 & 0.049 & $<.005$ & $<.005$ \\
\hline GS-4 & $1-7$ & 2205 & 88 & 2.245 & 0.019 & 0.026 & 0.154 & 0.027 & $<.005$ & $<.005$ \\
\hline GS -4 & $1-8$ & 150 & 92 & 2.309 & 0.019 & 0.022 & 0.107 & 0.026 & $<.005$ & $<.005$ \\
\hline GS-4 & $1-8$ & 620 & 99 & 2.146 & 0.022 & 0.015 & 0.089 & $<.005$ & $<.005$ & $<.005$ \\
\hline$G S+4$ & $1-8$ & 1020 & 103 & 2.130 & 0.011 & 0.011 & 0.071 & $<.005$ & 0.013 & $<.005$ \\
\hline GS - 4 & $1-8$ & 1417 & 107 & 2.175 & 0.008 & 0.010 & 0.071 & $<.005$ & $<.005$ & $<.005$ \\
\hline GS -4 & $1-8$ & 1805 & 114 & 2.129 & 0.010 & 0.012 & 0.055 & $<.005$ & $<.005$ & 0.006 \\
\hline GS-4 & 1.8 & 2205 & 118 & 2.186 & 0.013 & 0.009 & 0.066 & $<.005$ & 0.149 & $<.005$ \\
\hline GS-4 & $1-9$ & 209 & 125 & 2.042 & 0.013 & 0.010 & 0.056 & $<.005$ & $<.005$ & $<.005$ \\
\hline GS-4 & $1-9$ & 617 & 129 & 2.030 & 0.010 & 0.009 & 0.042 & $<.005$ & $<.005$ & $<.005$ \\
\hline GS -4 & $1-9$ & 1010 & 133 & 2.025 & 0.008 & $<.005$ & 0.051 & $<.005$ & $<.005$ & $<.005$ \\
\hline GS-4 & $1-9$ & 1410 & 140 & 2.066 & 0.008 & 0.006 & 0.093 & $<.005$ & $<.005$ & $<.005$ \\
\hline GS -4 & $1-9$ & 1808 & 144 & 2.028 & 0.013 & 0.010 & 0.074 & $<.005$ & 0.005 & $<.005$ \\
\hline GS -4 & $1-9$ & 2210 & 151 & 2.033 & 0.015 & $<.005$ & 0.097 & $<.005$ & $<.005$ & $<.005$ \\
\hline GS-4 & $1-10$ & 154 & 155 & 2.027 & 0.010 & 0.008 & 0.104 & $<.005$ & 0.012 & $<.005$ \\
\hline GS -4 & $1-10$ & 613 & 159 & 1.997 & 0.013 & 0.010 & 0.093 & $<.005$ & $<.005$ & $<.005$ \\
\hline GS -4 & $1-10$ & 1015 & 167 & 1.989 & 0.012 & $<.005$ & 0.081 & $<.005$ & $<.005$ & $<.005$ \\
\hline GS-4 & $1-10$ & 1407 & 171 & 2.013 & 0.009 & $<.005$ & 0.064 & $<.005$ & $<.005$ & 0.005 \\
\hline GS-4 & $1-10$ & 181.1 & 178 & 1.949 & 0.012 & 0.015 & 0.069 & $<.005$ & $<.005$ & $<.005$ \\
\hline GS-4 & $1-10$ & 1030 & 182 & 1.969 & 0.011 & $<.005$ & 0.051 & $<.005$ & $<.005$ & $<.005$ \\
\hline GS -4 & $1-11$ & 113 & 186 & 2.025 & 0.007 & 0.005 & 0.061 & $<.005$ & $<.005$ & $<.005$ \\
\hline GS -4 & $1-11$ & 610 & 193 & 2.211 & 0.029 & 0.011 & 0.178 & 0.071 & $<.005$ & $<.005$ \\
\hline GS-4 & $1-11$ & 1045 & 197 & 2.293 & 0.030 & 0.022 & 0.298 & 0.062 & $<.005$ & $<.005$ \\
\hline GS-4 & $1-11$ & 1418 & 204 & 2.249 & 0.141 & 0.066 & 0.322 & 0.102 & $<.005$ & $<.005$ \\
\hline GS -4 & $.1-11$ & 1835 & 377 & 2.413 & 0.050 & 0.017 & 0.484 & 0.147 & 0.013 & $<.005$ \\
\hline GS-4 & $1-11$ & 2225 & 212 & 2.306 & 0.027 & 0.038 & 0.514 & 0.131 & 0.011 & 0.126 \\
\hline GS -4 & $1-12$ & 150 & 219 & 2.352 & 0.047 & 0.025 & 0.544 & 0.124 & $<.005$ & 0.099 \\
\hline GS- 4 & $1-12$ & 614 & 223 & 2.448 & 0.048 & 0.018 & 0.622 & 0.144 & $<.005$ & $<.005$ \\
\hline GS-4 & $1-12$ & 1012 & 230 & 2.587 & 0.054 & 0.028 & 0.950 & 0.202 & $<.005$ & 0.121 \\
\hline GS -4 & $1-12$ & 1409 & 234 & 2.155 & 0.022 & 0.014 & 0.259 & 0.060 & $<.005$ & 0.068 \\
\hline GS-4 & $1-12$ & 1806 & 238 & 1.988 & 0.013 & 0.014 & 0.049 & $<.005$ & $<.005$ & $<.005$ \\
\hline GS -4 & $1-12$ & 2215 & 245 & 2.006 & $<.005$ & 0.009 & 0.037 & $<.005$ & $<.005$ & $<.005$ \\
\hline G5-4 & $1-13$ & 137 & 249 & 2.016 & 0.007 & $<.005$ & 0.035 & $<.005$ & $<.005$ & $<.005$ \\
\hline GS - 4 & $1-13$ & 621 & 256 & 2.021 & 0.017 & $<.005$ & 0.047 & $<.005$ & $<.005$ & $<.005$ \\
\hline GS -4 & $1-13$ & 1016 & 261 & 2.041 & 0.024 & 0.011 & 0.055 & $\leftarrow .005$ & $<.005$ & $<.005$ \\
\hline GS-4 & $1-13$ & 1418 & 265 & 2.078 & 0.019 & 0.013 & 0.117 & 0.037 & $<.005$ & $<.005$ \\
\hline GS -4 & $1-13$ & 1805 & 272 & 2.031 & 0.017 & $<.005$ & 0.072 & 0.015 & $<.005$ & 0.008 \\
\hline GS-4 & $1 \cdot 13$ & 2307 & 276 & 2.186 & 0.015 & 0.014 & 0.058 & $<.005$ & $<.005$ & $<.005$ \\
\hline GS -4 & $1-14$ & 210 & 283 & 1.986 & 0.007 & $<.005$ & 0.049 & $<.005$ & $<.005$ & $<.005$ \\
\hline GS -4 & $1-14$ & 622 & 287 & 2.010 & 0.015 & $<.005$ & 0.040 & $<.005$ & $<.005$ & $<.005$ \\
\hline GS-4 & $1-14$ & 1017 & 291 & 2.046 & 0.018 & 0.010 & 0.057 & $<.005$ & $<.005$ & $<.005$ \\
\hline GS -4 & $1-14$ & 1425 & 298 & 1.966 & 0.006 & 0.008 & 0.099 & 0.035 & $<.005$ & 0.009 \\
\hline GS-4 & $1-14$ & 1814 & 302 & 2.014 & 0.011 & 0.011 & 0.108 & $<.005$ & $<.005$ & $<.005$ \\
\hline GS -4 & $1-14$ & 2219 & 309 & 1.925 & $<.005$ & $<.005$ & 0.013 & 0.005 & $<.005$ & $<.005$ \\
\hline GS-4 & $1-15$ & 210 & 313 & 1.908 & $<.005$ & 0.007 & 0.012 & $<.005$ & 0.008 & $<.005$ \\
\hline & & 624 & 317 & 1.955 & 0.007 & 0.007 & 0.027 & $<.005$ & $<.005$ & $<.005$ \\
\hline
\end{tabular}


WESTINGHOUSE SAVANMAH RIVER SITE

- LANDFILL GROUNDSHEET STUDY -...

SOIL GAS CONCENTRATIONS (PPMV)

\begin{tabular}{|c|c|c|c|c|c|c|c|c|c|c|}
\hline SAMPLE & & & CYCLE & & & & & & ISO-BUTANE & N-BUTANE \\
\hline NAME & DATE & TIME & $\#$ & METHANE & ETHANE & ETHYLENE & PROPANE & PROPYLEAL & & \\
\hline GS -4 & & 1014 & 324 & 1.940 & $<.005$ & $<.005$ & 0.023 & 0.007 & $<.005$ & $<.005$ \\
\hline GS -4 & $1-15$ & 1417 & 328 & 1.938 & $<.005$ & 0.013 & 0.059 & $<.005$ & $<.005$ & $<.005$ \\
\hline & $1-15$ & 1815 & 335 & 1.866 & $<.005$ & 0.012 & 0.042 & $<.005$ & $<.005$ & $<.005$ \\
\hline
\end{tabular}

SAMPLES NOT COLLECTED DUE TO SURFACE HATER

GS- 4 1-15 2200

GS-4 $1-16 \quad 200$

$\begin{array}{lll}\text { GS }-4 & 1-16 \quad 1000\end{array}$

GS-4 $1-16 \quad 1430$

GS $-4 \quad 1-16 \quad 1804$

GS-4 $\quad 1-16 \quad 2206 \quad 36$

GS-4 $\quad 1-17 \quad 242 \quad 371$

GS-4 $\quad 1-17 \quad 618 \quad 375$

GS-4 $\quad 1-17 \quad 1021 \quad 385$

GS-4 $\quad 1-17 \quad 1430 \quad 389$

GS.4 $\quad 1-17 \quad 1810 \quad 393$

GS $4 \quad 1-17 \quad 2209 \quad 400$

GS-4 $1-18 \quad 150 \quad 404$

GS $-4 \quad 9-18 \quad 624 \quad 411$

GS $-4 \quad 1-18 \quad 1015 \quad 415$

GS-4 $\quad 1-18 \quad 1407 \quad 419$

GS $-4 \quad 1-18 \quad 1816 \quad 427$

GS-4 $1-18 \quad 2239 \quad 431$

GS-4 $\quad 1-19 \quad 203 \quad 438$

GS.4 $\quad 1-19 \quad 702 \quad 442$

GS-4 $\quad 1-19 \quad 1047 \quad 446$

1.851
1.876
1.942
1.892
1.916
1.861
1.924
1.910
1.906
1.895
1.894
1.918
1.893
1.890
1.896
1.854
1.899
1.881

$\begin{array}{ll}<.005 & <.005 \\ 0.006 & <.005 \\ 0.016 & <.005 \\ 0.020 & 0.013 \\ 0.018 & <.005 \\ <.005 & <.005 \\ 0.010 & 0.007 \\ 0.015 & 0.008 \\ 0.009 & <.005 \\ <.005 & 0.006 \\ <.005 & 0.010 \\ <.005 & 0.009 \\ 0.016 & <.005 \\ <.005 & 0.007 \\ <.005 & <.005 \\ <.005 & <.005 \\ 0.016 & 0.011 \\ 0.011 & <.005\end{array}$

0.048

$<.005$
0.008
$<.005$
$<.005$
$<.005$
$<.005$
$<.005$
0.029
$<.005$
$<.005$
0.023
$<.005$
0.006
$<.005$
$<.005$
$<.005$
$<.005$
0.005

0.010

$<.005$

0.042

0.020

$<.005$

$<.005$

0.025

$<.005$

$<.005$

0.033

$<.005$

$<.005$

$<.005$

GS-2A $\quad 1-5 \quad 1752$

15

413.999

0.057

$<.005$

12016.599

0.265

0.037

0.078

0.078

0.068

0.069

0.049

0.062

0.051

0.068

0.057

0.056

0.094

5003.481

0.210

0.044

0.035

34.735

0.013

$\begin{array}{llr}\text { GS-2A } & 1-7 & 1012 \\ \text { GS-2A } & 1-8 & 608\end{array}$

GS-2A $\quad 1-9 \quad 157$

10.112

$\begin{array}{lll}\text { GS-2A } & -1-9 & 2156 \\ \text { GS-2A } & 1-10 & 1802\end{array}$

121

180.609

$<.005$

0.005

0.188

0.037

$<.005$

$\begin{array}{lll}\text { GS-2A } & 1.11 \quad 1408\end{array}$

174

1901.128

0.011

$<.005$

0.021

$<.005$

0.006

14961.635

0.149

0.042

0.485

$<.005$

38.308

$<.005$

0.006

12.668

GS-2A $\quad 1-14 \quad 154 \quad 279$

$\begin{array}{llll}G S-2 A & 1-14 & 2207 & 305\end{array}$

$\begin{array}{llll}G S-2 A & 1-15 & 1806 & 332\end{array}$

311.340

0.012

$<.005$

53.815

0.027

$<.005$

$<.005$

324.231

2.685

0.005

0.005

$<.005$

$<.005$

20.746

0.010

$<.005$

63.968

$<.005$

$<.005$

$<.005$

2.484

0.017

0.009

2.367

0.020

0.019

2.089

0.021

$<.005$

2.027

0.009

2.007

0.010

$<.005$

2.611

0.377

2.013

0.363

0.049

$<.005$

0.196

0.390

$<.005$

3.011

0.061

3.981

$<.005$

0.474

0.054

0.028

0.014

$<.005$

0.204

0.015

0.026

0.078

$<.005$

$<.005$

0.031

0.033

0.068

$<.005$

$<.005$

$<.005$

0.083

0.155

$<.005$

$<.005$

0.255

0.053

$<.005$

$<.005$

$<.005 \quad 0.028$

$<.005<.005$

$<.005<.005$

$<.005<.005$

$<.005<.005$

$<.005<.005$

$<.005$

$<.005$

0.007

$<.005$

$<.005$

$<.005$

$<.005$

$<.005$

$<.005$

$<.005$

$<.005$

2.072

0.016

$<.005$

0.062

$<.005$

0.284

0.005

$\begin{array}{llll}G S-4 A & 1-11 & 115 & 188\end{array}$

2.260

0.032

0.018

$<.005$

$<.005$

4.057

$<.005$

3.284

0.701

$<.005$

$<.005$

$<.005$

$<.005$

$<.005$

$0.120<.005$

$0.576<.005$

$4.627 \quad 0.835$

GS $-4 A \quad 1-11 \quad 2227 \quad 213$

2.021

0.020

0.068

$<.005$

6.039

1.175

$<.005<.005$

$<.005<.005$

$0.300<.005$

$<.005<.005$

$<.005<.005$

$<.005<.005$

$0.009<.005$

$0.051<.005$

$<.005<.005$

$<.005<.005$

$<.005 \quad 0.105$

$<.005<.005$

$<.005<.005$

$<.005-<.005$

$<.005$
$<.005$

$<.005$

$<.005$

0.050

$<.005$

$<.005$

0.081

0.006

0.112

0.033

$<.005$

$<.005$ 
Table 1. (continued)

.... WESTINGHOUSE SAVANMAH RIVER SITE .....

.... LANDFILL GROUNDSHEET STUOY -...

..... SOIL GAS CONCENTRATIONS (PPMV) .....

\begin{tabular}{|c|c|c|c|c|c|c|c|c|c|c|}
\hline SAMPLE & & & :YCLE & & & & & PROPYLENE & ISO-BUTANE & N-BUTANE \\
\hline NAME & DATE & TIME & $\#$ & METHANE & ETHANE & ETHYLENE & PROPANE & & & \\
\hline$G S-4 A$ & $1-14$ & 1019 & 292 & 2.037 & 0.019 & $<.005$ & 0.061 & 0.008 & $<.005$ & $<.005$ \\
\hline GS-4A & $1-15$ & 626 & 318 & 1.953 & 0.007 & $<.005$ & 0.013 & 0.009 & $<.005$ & $<.005$ \\
\hline$G S-4 A$ & $1-16$ & 2208 & 365 & 1.958 & 0.008 & $<.005$ & 0.017 & $<.005$ & $<.005$ & $<.005$ \\
\hline$G S-4 A$ & $1-17$ & 1812 & 394 & 1.887 & 0.017 & $<.005$ & 0.063 & $<.005$ & $<.005$ & $<.005$ \\
\hline GS-4A & $1-18$ & 1411 & 421 & 1.872 & $<.005$ & $<.005$ & 0.043 & $<.005$ & $<.005$ & $<.005$ \\
\hline GS-4A & $1-19$ & 1048 & 447 & 1.860 & $<.005$ & $<.005$ & 0.032 & $<.005$ & $<.005$ & $<.005$ \\
\hline
\end{tabular}


Table 2 .

... WESTINGHOUSE SAVANKAH RIVER SITE -...

- LANDFILL GROUNOSHEET STUOY .....

STANOARD GAS CONCENTRATIONS (PPMV) ......

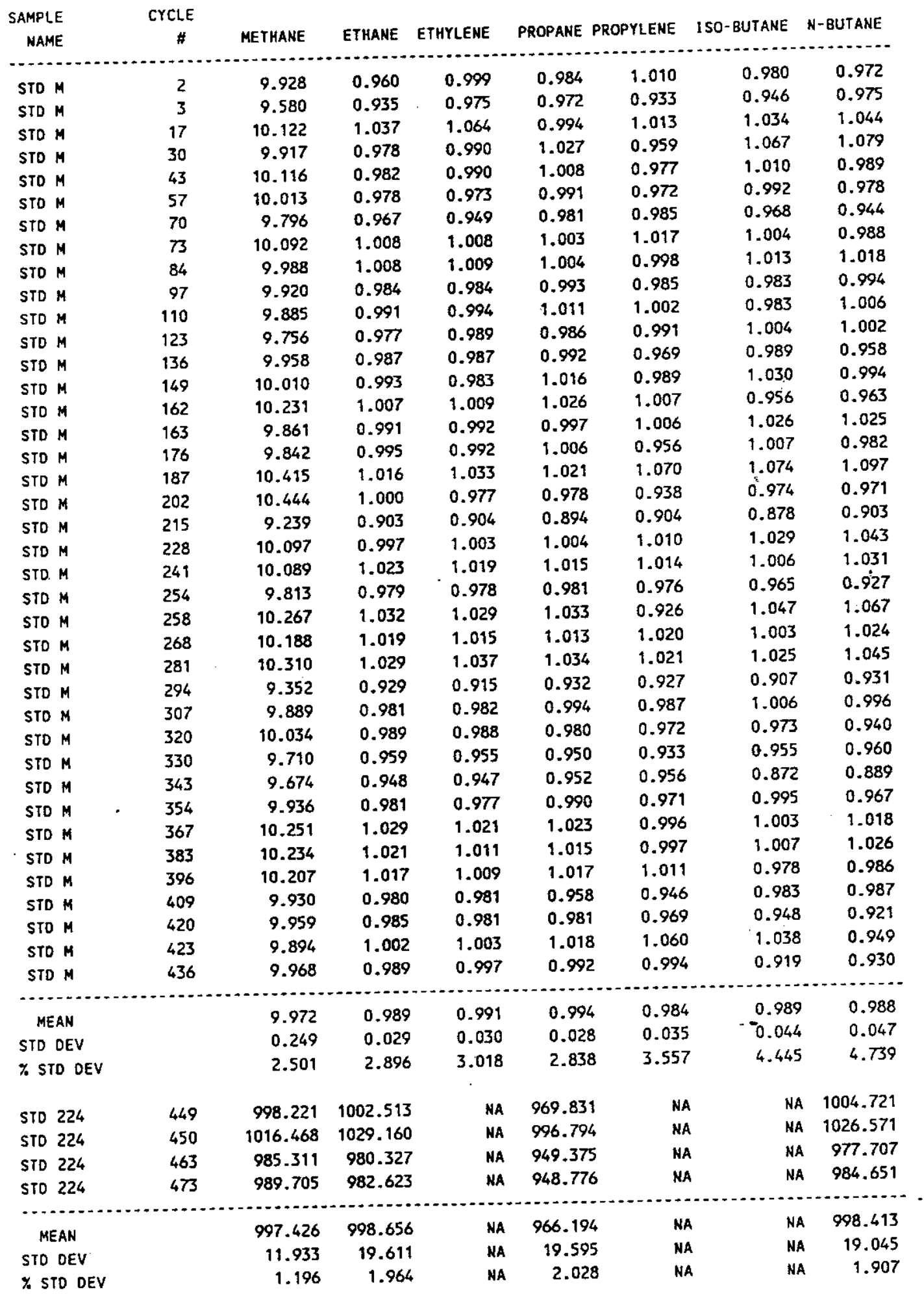

Table 2. Analyses of $\mathrm{Cl}-\mathrm{C} 4$ Hydrocarbon standard " $M$ " and standard "224" During the 740-G Landfill Groundsheet study 
Table 3 .

WESTINGHOUSE SAVANMAH RIVER SITE -...

.. LANDFILL GROUNDSHEET STUDY .....

.... SYSTEM BLANK CONCENTRATIONS (PPMV)

\begin{tabular}{|c|c|c|c|c|c|c|c|c|c|c|}
\hline \multirow{2}{*}{$\begin{array}{r}\text { SAMPLE } \\
\text { NAME }\end{array}$} & \multicolumn{4}{|c|}{ CYCLE } & \multirow[b]{2}{*}{ ETHANE } & \multirow{2}{*}{ ETHYLENE } & \multirow{2}{*}{\multicolumn{2}{|c|}{ PROPANE PROPYLENE }} & \multirow{2}{*}{ ISO-BUTANE } & \multirow{2}{*}{ N-BUTANE } \\
\hline & DATE & TIME & $\#$ & METHANE & & & & & & \\
\hline GS-BLKHI & $1-5$ & 1000 & 4 & 2.034 & $<.005$ & $<.005$ & $-<.005$ & $<.005$ & $<.005$ & $<.005$ \\
\hline GS-BLKH2 & $1-5$ & 1755 & 16 & 2.059 & 0.039 & 0.073 & $<.005$ & $<.005$ & $<.005$ & $<.005$ \\
\hline GS-BLK\#3 & $1-6$ & 216 & 29 & 2.067 & 0.021 & 0.014 & 0.016 & $<.005$ & $<.005$ & $<.005$ \\
\hline GS-BLK\#4 & $1-6$ & 1402 & 42 & 3.701 & 0.023 & 0.007 & 0.007 & $<.005$ & $<.005$ & $<.005$ \\
\hline GS-8LX\#S & $1-6$ & 2217 & 56 & 2.145 & 0.014 & 0.008 & $<.005$ & 0.008 & $<.005$ & $<.005$ \\
\hline GS-BLK\#6 & $1-7$ & 1015 & 69 & 2.302 & 0.010 & 0.012 & $<.005$ & $<.005$ & $<.005$ & $<.005$ \\
\hline GS-BLK\#7 & $1-7$ & 1814 & 83 & 2.014 & 0.010 & $<.005$ & $<.005$ & 0.008 & $<.005$ & $<.005$ \\
\hline GS-BLK\#8 & $1-8$ & 610 & 96 & 2.089 & 0.012 & $<.005$ & 0.023 & $<.005$ & $<.005$ & $<.005$ \\
\hline GS-BLX\#9 & $1-8$ & 1421 & 109 & 2.060 & 0.012 & $<.005$ & $<.005$ & 0.017 & $<.005$ & $<.005$ \\
\hline GS-BLK\#10 & $1-9$ & 159 & 122 & 2.103 & 0.019 & 0.006 & $<.005$ & $<.005$ & 0.006 & $<.005$ \\
\hline GS-8LK\#11 & $1-9$ & 1015 & 135 & 2.026 & 0.008 & 0.005 & 0.011 & $<.005$ & $<.005$ & $<.005$ \\
\hline GS-BLK\#12 & $1-9$ & 2157 & 148 & 1.990 & 0.009 & $<.005$ & $<.005$ & $<.005$ & $<.005$ & $<.005$ \\
\hline GS-BLK\#13 & $1-10$ & 618 & 161 & 2.049 & 0.007 & $<.005$ & 0.087 & $<.005$ & $<.005$ & $<.005$ \\
\hline GS-BLX\#14 & $1-10$ & 1804 & 175 & 2.067 & 0.007 & $<.005$ & $<.005$ & $<.005$ & $<.005$ & $<.005$ \\
\hline GS BLK\#15 & $1-11$ & 117 & 189 & 2.084 & 0.065 & $<.005$ & $<.005$ & $<.005$ & $<.005$ & $<.005$ \\
\hline GS BLK\#16 & $1-11$ & 1410 & 201 & 3.645 & 0.025 & $<.005$ & 0.010 & $<.005$ & $<.005$ & $<.005$ \\
\hline GS-BLK\#17 & $1-11$ & 2229 & 214 & 2.057 & 0.007 & $<.005$ & $<.005$ & $<.005$ & $<.005$ & $<.005$ \\
\hline GS-BLK\#18 & $1-12$ & 1005 & 227 & 3.653 & 0.010 & $<.005$ & 0.007 & $<.005$ & $<.005$ & $<.005$ \\
\hline GS-BLX\#19 & $1-12$ & 1813 & 240 & 2.004 & 0.015 & 0.026 & $<.005$ & $<.00 ॄ 5$ & $<.005$ & 0.008 \\
\hline GS-BLK\#20 & $1-13$ & 615 & 253 & 2.008 & $<.005$ & $<.005$ & $<.005$ & 0.005 & $<.005$ & $<.005$ \\
\hline GS-BLX\#21 & $1 \cdot 13$ & 1422 & 267 & 2.043 & 0.024 & $<.005$ & $<.005$ & $<.005$ & $<.005$ & 0.008 \\
\hline GS-BLK\#22 & $1-14$ & 200 & 280 & 2.003 & 0.005 & 0.013 & $<.005$ & $<.005$ & $<.005$ & $<.005$ \\
\hline GS- $8 L K \$ 23$ & $1-14$ & 1021 & 293 & 1.985 & 0.005 & $<.005$ & $<.005$ & $<.005$ & $<.005$ & 0.024 \\
\hline GS-BLK $\# 24$ & $1-14$ & 2209 & 306 & 1.962 & 0.020 & 0.008 & $<.005$ & $<.005$ & 0.007 & $<.005$ \\
\hline GS-BLK\$2S & $1-15$ & 629 & 319 & 2.031 & 0.029 & 0.095 & $<.005$ & 0.071 & $<.005$ & 0.125 \\
\hline GS-BLK\#26 & $1-15$ & 1808 & 333 & 2.188 & $<.005$ & $<.005$ & $<.005$ & $<.005$ & $<.005$ & $<.005$ \\
\hline GS-BLK\#27 & $1-16$ & 200 & 342 & 1.787 & 0.014 & $<.005$ & $<.005$ & $<.005$ & $<.005$ & $<.005$ \\
\hline GS-BLK\#28 & $1-16$ & 1410 & 353 & 1.908 & 0.006 & $<.005$ & 0.014 & $<.005$ & $<.005$ & $<.005$ \\
\hline GS-BLK\#29 & $1-16$ & 2210 & 366 & 1.936 & $<.005$ & $<.005$ & 0.010 & $<.005$ & $<.005$ & $<.005$ \\
\hline GS-BLX\$30 & $1-17$ & 1016 & 382 & 1.894 & 0.012 & $<.005$ & $<.005$ & $<.005$ & $<.005$ & $<.005$ \\
\hline GS-BLK\#31 & $1-17$ & 1813 & 395 & 1.880 & $<.005$ & $<.005$ & 0.008 & $<.005$ & $<.005$ & $<.005$ \\
\hline GS-BLK $\# 32$ & $.1 \cdot 18$ & 618 & 408 & 1.904 & $<.005$ & 0.010 & $<.005$ & $<.005$ & $<.005$ & $<.005$ \\
\hline GS-8LK\#33 & $1-18$ & 1412 & 422 & 1.874 & $<.005$ & $<.005$ & $<.005$ & $<.005$ & $<.005$ & $<.005$ \\
\hline GS-BLX $\# 34$ & $1-19$ & 154 & 435 & 1.876 & 0.012 & 0.014 & $<.005$ & $<.005$ & $<.005$ & $<.005$ \\
\hline GS-BLK\#35 & $1-19$ & 1051 & 448 & 1.843 & $<.005$ & $<.005$ & $<.005$ & $<.005$ & $<.005$ & $<.005$ \\
\hline & & & & & & & & & & \\
\hline & MEAN & & & 2.151 & 0.013 & 0.011 & 0.009 & 0.007 & 0.005 & 0.009 \\
\hline & STD DEV & & & 0.475 & 0.012 & 0.019 & 0.014 & 0.011 & 0.000 & $\begin{array}{l}0.020 \\
0.049\end{array}$ \\
\hline & DEV & & & 3.100 & 0.037 & 0.049 & 0.037 & 0.030 & 0.006 & 0.049 \\
\hline
\end{tabular}

Table 3. Analyses of System Blanks 
Table 4 .

MICROSEEPS

$\star \star 02-A p r-91 * *$

WESTINGHOUSE SAVANNAH RIVER SITE-.................

.... 740-G LANDFILL ....

....-LIGHT HYDROCARBON SOIL GAS CONCENTRATIONS a 3 FT-......

\begin{tabular}{|c|c|c|c|c|c|c|c|c|c|c|}
\hline & & & & & 12461 & 19042 & 260 & 2088 & 2927 & $740-G$ \\
\hline $740-6$ & 52 & 38.3 & 382810000 & ND & 25926 & 34516 & 7929 & 2403 & 3404 & $740-G$ \\
\hline $740-6$ & 97 & 56.4 & 563750000 & 176420 & 297700 & 141170 & 202710 & 46632 & 73436 & $740-G$ \\
\hline $740-G$ & 220 & 26.1 & 260900000 & 220490 & 683020 & 130170 & 169070 & 9002 & 6595 & $740-G 220$ \\
\hline
\end{tabular}

Table 4. Light Hydrocarbon Concentrations in soil Gas Survey Sites Near Each Groundsheet 
This page intentionally left blank. 


\section{Appendix I}

\section{Sampling Method SM1}

Analytical Method AMI 
This page intentionally left blank. 


\title{
SAMPLING METHOD SMI
}

\author{
SOIL GAS PROBE SAMPLING
}

\subsection{Scope and Application}

1.1 Soil gas probe samples are collected with Microseeps unique, portable soil gas sampling probe which permits access to any area. In addition, the probe allows unlimited purging between sample sites and incorporates the capability to discard the volume of air in the sample probe before the soil gas sample at each site is taken.

\subsection{Apparatus}

2.1 Soil Gas Probe: The soil gas probe consists of a $1 / 2$ inch diameter steel tube equipped with a rubber packer and a $1 / 8$ " $0 . d$. ss sample tube inserted concentrically to the bottom of the $1 / 2$ " diameter tube to minimize dead space in the sample volume. The $1 / 8$ " o.d. sample tube is connected through a three way valve to a 250 cc gas tight syringe mounted as an integral part of the sample probe. The third port of the three-way valve is terminated with a syringe needle and serves two functions: first a small amount of gas equal to or larger than the dead space volume of the 1/8" o.d. sample tube is discarded through this port before each soil gas sample is taken; second, the syringe needle serves to facilitate transfer of the soil gas sample through a rubber septum into a previously evacuated sample vial, minimizing the possibility of contamination of any sample.

2.2 slide Hammer Plunger Bar: The slide hammer plunger bar contains a $5.5 \mathrm{ft}$. $x .5$ in rod with a $9 / 16$ inch ball. Fitted over one end of the rod is a weighted slide hammer. (Heath Consultants cat\# 478).

2.3 Sample vials: The sample vials used are determined by the analytical method that is required for analysis. All vials should be free of interferences and meet the specifications described in the analytical method to be used.

\subsection{Procedure}

3.1 Prior to any sampling, the soil gas sampling probe should be purged clean of interferences, the syringe needle should be removed, and the three way valve should be in the stop flow position.

3.2 A $9 / 16$ inch diameter hole is created to the desired depth using the slide hammer plunger bar. 
3.3 The plunger bar is removed and the sample probe is inserted into the hole. A seal should be obtained to prevent ambient air from entering the hole when the soil gas sample is removed.

3.4 The three way valve is turned to the probe/syringe position.

3.5 A $10 \mathrm{cc}$ volume of gas is drawn into the syringe.

3.6 The three way valve is turned to the syringe/needle position (needle removed as described in section 3.1 ) and the 10 cc sample is discarded.

3.7 The three way valve is turned to the probe/syringe position and the appropriate amount of soil gas is drawn into the syringe.

3.8 The three way valve is turned to the stop flow position

3.9 The needle is attached to the probe needle fitting.

3.10 An evacuated sample vial is attached by inserting the sample vial septum onto the needle.

3.Il The three way valve is turned to the syringe/needle position. The volume of the evacuated vial will be drawn into the sample vial. Additional volume (positive pressure) can then be added by depressing the plunger and displacing the remaining volume in the syringe.

3.12 The sample vial should be quickly removed once desired volume is displaced.

3.13 A volume of approximately $50 \mathrm{cc}$ of ambient air should be drawn back through the needle.

3.14 Remove the needle.

3.15 Remove the sample probe from the hole.

3.16 Purge the sample probe clean of interferences.

\subsection{Sample Documentation}

4.1 All samples should be labeled immediately after collection with the following information:

1. Site number

2. Daily sequence number

3. Date and time

4. Samplers initials

5. Project 
4.2 Al samples taken in the field by Microseeps, Ltd. should be entered onto a Field Log sheet. For each sample, the following entries will be made:

1. Site number

2. Daily sequence number

3. Date and time

4. Samplers initials

5. Project

6. Location

7. Sample description

8. Miscellaneous comments

4.3 When appropriate, samples will be security sealed and chain of custody records will be maintained.

\subsection{Safety Precautions}

5.I Do not under any circumstances allow the pressure in the sampling syringe or sample vial to exceed 20 psig. Operations above 20 psig may cause the glass to burst which could possibly result in injury to the user or bystanders. Do not use a mechanical device to depress the sampling syringe plunger.

5.2 Do not attempt to collect soil gas probe samples without prior knowledge of the location of underground utilities and other possible environmental hazards.

5.3 Always cap or remove the soil gas probe syringe needle when transporting or handling the soil gas probe.

\subsection{Preventive Maintenance}

6.0 The soil gas sample probe should be cleaned when necessary or at the end of each day with soap and organic free water. It is especially important to keep the gas sampling syringe clean from fine soil particles as the particles will quickly deteriorate the teflon/glass plunger seal.

6.I The glass wool filter plug (located in the probe tip) should be changed often and the probe tip should be kept clear of soil. 


\author{
ANALYTICAL METHOD AMI
}

\author{
ANALYSIS OF CI-C4 HYDROCARBONS, HYDROGEN, HELIUM, \\ IN SOIL GAS
}

\title{
1.0 Scope and Application
}

1.I Method $\mathrm{AMl}$ is used to determine the concentration of $\mathrm{Cl}-\mathrm{C} 4$ hydrocarbons, helium and hydrogen in soil gas samples. Specifically. Method AMI may be used to detect the following substances:

methane

ethane

ethylene

propane

propy lene

i-butane

n-butane

hel i um

hydrogen

1.2 This method is recommended for use by, or under the supervision of analysts experienced in the operation of gas chromatographs and in the interpretation of chromatograms.

\subsection{Summary of Method}

2.I Analysis of the above constituents of free soil gas is accomplished with a custom chromatograph, built by Microseeps. The accomplished with a custom chromatogras provides a- sequential output
custom, dual detector gas chromatograph provith a
from two detectors. First, the Cl-C4 hydrocarbons are detected with flame ionization detector (FID), next helium and hydrogen are detected with a thermal conductivity detector (TCD). The sample (and standard calibration gas) is flowed through the gas chromatograph and introduced into the columns by the mechanical injection of two sample loops.

\subsection{Interferences}

3.1 Contamination by carryover can occur whenever high-level and lowlevel samples are sequentially analyzed. A non-restricted flow of pure nitrogen from a 10 psig source should be purged for 30 seconds through the sample loops prior to all analyses.

3.2 The analyst should demonstrate the absence of contamination by carryover by analysis of the contents of the sample loops when purged with nitrogen. This demonstration should be performed prior to the analysis of a sample set and when carryover contamination is suspected 
(after high samples). In the event that 'ghost peaks' (peaks similar to previous sample) appear when a pure nitrogen sample is analyzed. measures should be taken to eliminate the carryover contamination.

3.3 Extra peaks in a chromatogram can be actual peaks from a previous run. Contamination from late eluting peaks can occur when injection to injection time is too short. The dual gas chromatograph is set up in the backflush pre-column valve arrangement to minimize this interference.

3.4 The analyst should be certain that all peaks have eluted from the previous analysis prior to analyzing any sample or standard. If samples or standard chromatograms contain suspected 'extra peaks' the sample should again be analyzed after a clean baseline is established.

\subsection{Apparatus and Materials}

4.1 Sample vials: $125 \mathrm{ml}$ glass vials (Wheaton \#223748 or equivalent). vials should be free of hydrocarbons, helium, and hydrogen prior to use. This can be accomplished by heating to 100 degrees $C$ followed by purging with pure nitrogen.

4.2 Septums: Butyl rubber septums (Wheaton \#224154 or equivalent) may be used provided vials are capped within two weeks prior to use. other septums may be used, provided they are gas tight and do not produce interferences.

4.3 Gas Chromatograph: The chromatograph is equipped with two sets of the following: column oven, pre-column, analytical column, detector, injection port, sample valve and sample loop. The column and detector for determination of $\mathrm{Cl}-\mathrm{C4}$ hydrocarbons are a granular $3 \mathrm{ft}$. $x$ 3/16 in. alumina analytical column and a flame ionization detector. The alumina column is protected against contamination by heavy organics by a 3 in. $x$ 3/16 in. pre-column which is back-flushed after butanes have entered the analyticial column. This arrangement allows rapid turn-around for consecutive analyses and a clean baseline for accurate, reproducible results. The flame ionization detector is of a special design which allows considerably more sensitivity than commercially available models. In a laboratory setting, noise levels are commonly at the 500 ppt level. Although, these sensitivities are rarely realized in the field. 1 to 2 $\mathrm{ppb}$ is achievable and $5 \mathrm{ppb}$ is routine. Hydrogen and helium, are simultaneously determined using a 3 ft. $x 3 / 16$ in. molecular sieve $5 \mathrm{~A}$ $(80 / 100$ mesh) pre-column and $10 \mathrm{ft} . \mathrm{x} 3 / 16 \mathrm{in}$. analytical column connected to a thermal conductivity detector. Injection times and the signal output are sequenced so that the output of the two detectors is displayed in a single continuous chromatogram.

4.4 Data Collection: The output of the chromatograph is directed to a chart recorder and a Hewlett Packard (HP-3392A) Networking Integrator which passes the processed data to a microcomputer for data storage and/or further processing.

5.0 Sample Preparation and Analysis 
5.1 Sample vial preparation: All sample vials should meet specifications as noted in sections 4.1 and 4.2 above. Vials should be tightly capped and evacuated to a pressure of less than 100 millitorr. The vial septum should be punctured with needles of 22 gauge or smaller.

5.2 The evacuated sample vials should be filled with sample gas to a pressure of 9 psig. A positive pressure capable of delivering at least 25 cc of sample must be available per each injection.

5.3 The pressurized sample vial is connected to the sample loops through a needle fitting and flow control valve. The fiow is monitored by a flow meter connected to the out port on the gas chromatograph.

5.4 After the appropriate volume of sample (minimum $25 \mathrm{cc}$ ) has been flushed through the sample loops of the gas chromatograph, the injection values may be activated. This is accomplished by switching on the cycle timer.

\subsection{Calibration}

6.1 The standard calibration gas should be introduced in the same manner as described in section 5.4 above. Measured peak areas are converted to concentrations in parts per million by volume using certified commercial gas standards traceable to NBS standards.. (Matheson Gas Products Inc., or scott specialty Gases). The sample concentrations are calculated from calibration points near the concentration level of the sample.

6.2 At the beginning of a project or sample set, standards of appropriate calibration ranges will be run at least three times or until the results agree with a percent standard deviation no greater than $10 \%$.

6.3 The instrument response (for any one subsequent standard in section 6.5 above) must not vary by more than 20\%: or any two consecutive standards must not vary by more than $15 \%$ from the mean of the three previous standards. 


\subsection{Quality Control}

7.1 If the parameters set forth in section 6.3 are not met, the analytical program will be terminated until the cause is determined and a solution is effected. The cause and the solution will be recorded in the Laboratory Notebook and signed by the operater, before the analytical program is resumed.

7.2 Before and during sample analysis, instrument blanks (sample loops filled with flush nitrogen) should be analyzed to assure the absence of interferences as described in section 3.0 above.

7.3 standards analyzed during the course of analyzing samples are averaged into the calibration table as well as being used for peak identification. All chromatograms should be examined by an experienced analyst.

7.4 Throughout analysis the soil gas samples are injected mechanically to achieve a uniform sample size from a flow directly from the soil gas sample vial which has been pressured at the time of sampling. This pressuring preserves sample integrity since any leakage is out of the vial and does not result in contamination or sample dilution.

7.5 Calibration records are generated and stored. All such records will be maintained in the laboratory during the course of the project.

\subsection{Instrument Conditions}

8.1 Gas Chromatograph:

Injection Temp. ambient

Flame Ionization Detector Temp. ambient

Thermal Conductivity Detector Temp. 45 deg. C.

C1-C4 oven Temp. 100 deg. C. isothermal

Helium/Hydrogen Oven Temp. $45 \mathrm{deg}$. C. isothermal

Initial T.C.D. Signal Range 10

Initial F.I.D. Signal Range loE9

Carrier Gas Regulator Pressures:

T.C.D. 40 psig.

F.I.D. 17 psig.

Hydrogen Pressure 22 psig.

Flame Air Pressure 30 psig. ( $1.0 \mathrm{scfh}$ ) 
This page intentionally left blank. 
Appendix II

Soil Gas Collection Logs 
aIEH: W.S.R.C

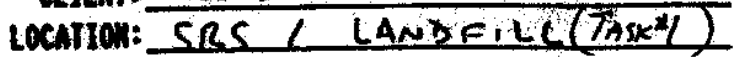

PraJECT( 1): $L A \rightarrow D$ EICL GRUUNDSLRET

PAGE: $\angle$ of:

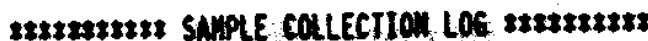

SAMPLER Mane(S): DM-AGC-JS-JP-ROP

\begin{tabular}{|c|c|c|c|c|c|c|c|c|c|c|}
\hline SAMPLE IDI & DATE & TIME & SEO.1 & SAMPLE & & XE & IYPE & SMPLE & COMHERTS & \\
\hline 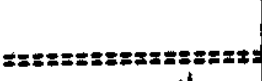 & $2=2: 2 x=z=$ & & & $\begin{array}{r}\text { DEPTH } \\
=x=2 x= \pm x=\end{array}$ & $=8$ & 5 & 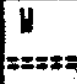 & SIIE & 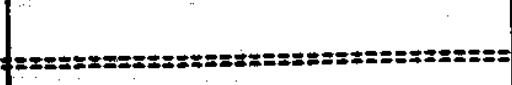 & C.OC: \\
\hline$G S-B I K+1$ & $1-5-91$ & $10: 00$ & $j$ & $-\quad$ & $\checkmark$ & & & $22 \mathrm{ml}$ & Sustem Bianh & $|-5-4|$ \\
\hline $6 s-1$ & $1-5$ & 10.02 & 2 & $Q^{\prime}$ & $f$ & & & & 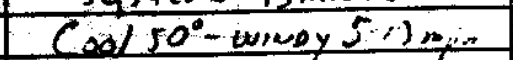 & 2 \\
\hline$G S-2$ & 1.5 & $10: 12$ & 3 & & & & & $i$ & & ? \\
\hline$G S-3$ & 1.5 & $10: 20$ & 4 & & & & & & & \\
\hline$G S-4$ & $1-5$ & 10.28 & 5 & & & & & 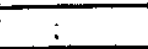 & & \\
\hline$G s-1$ & $1-5$ & 13.58 & 6 & & & & & & & i \\
\hline$C S-2$ & $1-5$ & $14: 05$ & 7 & & $!$ & & & 1 & & \\
\hline$G S-3$ & $1-5$ & $4: 10$ & 3 & & & & & 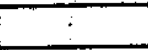 & & \\
\hline $65-4$ & $1-5$ & $14: 15$ & 9 & & & & & & • & $\frac{1}{1}$ \\
\hline $65-1$ & $1-5$ & $17: 45$ & 10 & & & & & $i$ & & 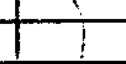 \\
\hline $6 S-2$ & $1-5$ & $12: 50$ & 11 & & $\bar{T}$ & & & $!$ & & 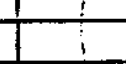 \\
\hline$G 5-2 A$ & $1-5$ & $17: 52$ & 12 & & & & & $\therefore$ & Duplicate SAmp'e & 1 \\
\hline GS-BIK 2 & $1-5$ & 12.55 & $\sqrt{3}$ & & & & & 1 & Surtem Blank & $\frac{1}{1}$ \\
\hline $65-3$ & $1-5$ & $18: 00$ & 14 & & $i$ & & & $!$ & & 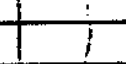 \\
\hline$G 5-4$ & $1-5$ & $18: 10$ & 15 & & & & & & & \\
\hline Gs-1 & -2 & $22: 20$ & 16 & & & & & & DARh BuL CALM & + \\
\hline$G S-2$ & $1-5$ & $22: 25$ & 12 & & & & & & $\cdots$ & \\
\hline $65-3$ & $1-5$ & $22: 30$ & 18 & & & & & & $\because$ & 1 \\
\hline $65-4$ & $1-5$ & $22: 35$ & 79 & & & & & & & IAGC. \\
\hline $65-1$ & $\therefore$ & 96 & 20 & & & & & & v. derk & $12-6-9$ \\
\hline $65 \cdot 2$ & $\ddot{r}$ & $\because 22$ & $2 i$ & & & & & & $\because$ & 1 \\
\hline$G 5-3$ & $\because$ & $\cdots$ & $z-$ & & & & & & 11 & $\therefore$ \\
\hline $65-4$ & $\therefore$ & $2: 5$ & 23 & & & & & & 1 & \\
\hline $65-4 A$ & $-\because$ & $2 \%$ & 24 & & & & & & Dup. & \\
\hline$C S-B I K^{2} 3$ & & $\because \div$ & 25 & & & & & & $31 k \quad$ : & $!$ \\
\hline $65-1$ & $1-6$ & 5607 & $\because 5$ & & & & & & 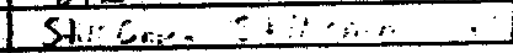 & $i$ \\
\hline GS.2 & $1-6$ & $x / 0$ & $2 y$ & & & & & & & 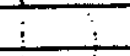 \\
\hline $6 s-3$ & 1.6 & $C 615$ & 3 & & & & & & & 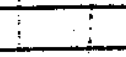 \\
\hline $6 s-4$ & $i-h$ & 0621 & 20 & & & & & & & . \\
\hline $6 c-1$ & $1-6$ & $10: 0^{2}$ & $\therefore$ & & & & & & $\mathrm{Cos}_{2}-\mathrm{n} \mathrm{nim}$ & $\vdots$ \\
\hline $65-2$ & $\therefore:$ & $20 \div 3$ & 1 & & & & & & & \\
\hline $6 \div-6$ & 6 & $10: 15$ & 3 & & & & & & & \\
\hline $6 ;-6$ & $i s$ & $10: 29$ & 26 & & & & & & & \\
\hline $6 s-1$ & $\%$ & $13: 50$ & 34 & & & & & & Sunwy inaren & \\
\hline $6 s-6$ & 3 & 13.55 & 35 & & & & & & & \\
\hline$\therefore \therefore H$ & $\therefore$ & 1400 & 36 & & & & & & Oupleate Sample & . \\
\hline$\epsilon^{\prime}: 3$ & 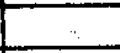 & $(4)^{\prime}$ & 7 & & & & & & Systen Blank & \\
\hline $6 \div ?$ & & 1405 & 33 & & & & & & & \\
\hline$\therefore \div$ & & i, & 21 & & & & & & & \\
\hline $65-1$ & $\therefore 9$ & $1 \%:$ & $\because 3$ & & & & & & $\therefore \ldots$ & \\
\hline$G S-2$ & $j-6$ & $1 / 806$ & 41 & & & & & & & \\
\hline $6-3$ & 7.7 & $1,1: 2$ & $\because$ & & & & & & & \\
\hline $65-4$ & $7-2$ & 11 & & & & & & & & \\
\hline
\end{tabular}

USE A NEL SHEE: "WEN RESTARTINE SEOUENCE I 
MICROSEEPS LTD.

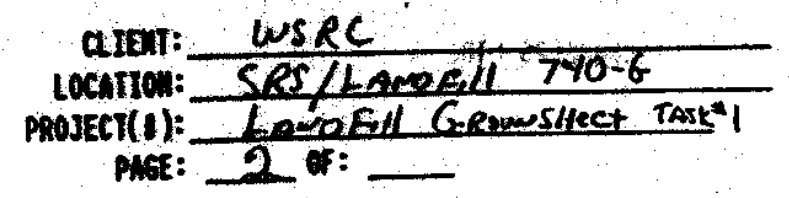

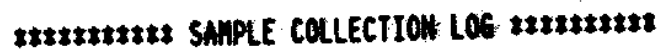

SAMPLER MAME(S): DJM, $A G C, R J P$

\begin{tabular}{|c|c|c|c|c|c|c|c|c|c|}
\hline \multirow[t]{2}{*}{ SAMPLE IOI } & \multirow[t]{2}{*}{ OATE } & \multirow[t]{2}{*}{ TInE: } & \multirow[t]{2}{*}{ SEO.1 } & \multirow{2}{*}{$\begin{array}{l}\text { SAMPLE } \\
\text { DEPTH }\end{array}$} & \multicolumn{3}{|c|}{ SAMPLE TYPE } & \multirow{2}{*}{$\begin{array}{r}\text { SAMPLE } \\
\text { SIZE }\end{array}$} & Contits \\
\hline & & & & & $\begin{array}{c}6 \\
2 x=2 z\end{array}$ & 5 & 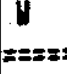 & & 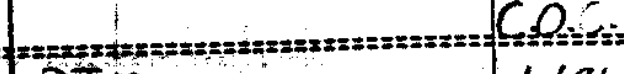 \\
\hline$\frac{C S-1}{G S-2}$ & $1-6-91$ & 22.00 & 44 & $0^{\prime}$ & $\checkmark$ & & & $22 \mathrm{~mL}$ & DJn $\quad|-6-9|$ \\
\hline$\frac{G S-2}{G S-3}$ & $1-6$ & $22: 05$ & 45 & 1 & 1 & & & & $1+4$ \\
\hline$\frac{G S-3}{G S-4}$ & $1-6$ & $22: 10$ & 46 & 1 & & & & 1 & +1 \\
\hline$\frac{C S-4}{G S-4 A}$ & $1-6$ & $22: 15$ & 47 & 1 & & & & $t$ & 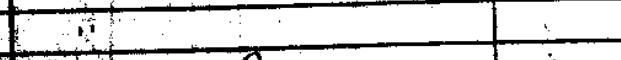 \\
\hline$\frac{G S-4 A}{6 S-B \operatorname{lank}=5}$ & $1-6$ & $22: 16$ & 48 & & & & & & Dup \\
\hline $\begin{array}{l}65-B \text { lank } \\
65-1\end{array}$ & $1-6$ & $22: 17$ & 49 & & & & & & $S Y S-B L K$ \\
\hline$\frac{65-1}{65 \cdot 2}$ & $1-7$ & $\$ 1=6$ & 50 & & & & & & $24 p$ \\
\hline$\frac{65 \cdot 2}{65 \cdot 3}$ & $1-7$ & $0:-2$ & 51 & & & & & & 1 \\
\hline$\frac{G 5 \cdot 3}{G 5 \cdot 4}$ & $1-7$ & 0 & 52 & & & & & & i \\
\hline$\frac{G S \cdot 4}{G S-1}$ & $1-7$ & $0: 2$ & 33 & & & & & & ap C \\
\hline$\frac{65-1}{65 \cdot 2}$ & $1-7$ & 060 & 34 & & & & & & $A G C-L g h+\alpha+n-2$ arse ioc \\
\hline$\frac{65 \cdot 2}{65 \cdot 3}$ & $1-2$ & 2610 & $55^{2}$ & & & & & $\cdot$ & $55-60^{3}-C A L M$ \\
\hline$\frac{65-3}{G 5 \cdot 4}$ & $1-7$ & 2015 & 52 & $\vdots$ & & & & & \\
\hline$\frac{G S \cdot 4}{G S-1}$ & $\angle-7$ & $06 \div 2$ & 57 & 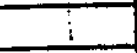 & & & & & \\
\hline$\frac{G S-1}{G-2}$ & $\therefore 1$ & 1005 & 58 & 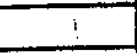 & & & & & Calm \\
\hline$\frac{G S .2}{G S .2 A}$ & $\cdots$ & 1212 & 54 & $\vdots$ & & & & & 管 \\
\hline$G 5 \cdot 2 A$ & $i 2$ & 1012 & 60 & $\bar{\vdots}$ & & & & & Dep \\
\hline$(x-5-8)_{a x+6}$ & $\theta$ & $10 / 5$ & 21 & & & & & & Sus ale \\
\hline $65-3$ & $\therefore \%$ & 1020 & 62 & & & & & & \\
\hline-4 & $\cdots$ & $k^{2} 25$ & 63 & 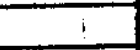 & & & & & $:$ \\
\hline 65.1 & $1-2$ & $\therefore, 5$ & 34 & $!$ & & & & & Curnoy+Rangs \\
\hline 65.2 & $\not \cdot 7$ & $\because$ & 65 & 1 & & & & & \\
\hline $6<.3$ & $2-7$ & in & 06 & $i$ & & & & & \\
\hline $6.5 \cdot 4$ & $V-i$ & .1 & 67 & & & & & & \\
\hline$G 5-1$ & $\angle-2$ & 1755 & 68 & & & & & & $C A l_{m}+P_{A 1 N}$ \\
\hline $6,5: 2$ & $1-2$ & .1325 & 19 & & & & & & \\
\hline 65.3 & $L-2$ & $70^{\circ}$ & in & & & & & & \\
\hline 65.4 & $1-7$ & $\because 6$ & $Z$ & & & & & & \\
\hline$C 5.4 A$ & $1-7$ & $\pi /:$ & 72 & & & & & & Que \\
\hline CS-Blonk? & $1-7$ & 2714 & 23 & & & & & & SysRuK \\
\hline G? -1 & $1-7$ & $2 ! \cdot 50$ & 3 & & & & & & Ninber con! \\
\hline $55-$ & $1-\infty$ & $2: \vdots$ & $\because$ & & & & & & $\begin{array}{ll}11 \\
\end{array}$ \\
\hline 65. & $1-9$ & 2200 & 26 & & & & & 7 & $\because$ \\
\hline 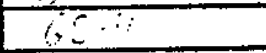 & $\therefore-3$ & $=2: 5$ & $7 \%$ & : & & & & & $=28$ \\
\hline $5-i$ & $1-8$ & $\because 1=7$ & 0 & & & & & & Fininger \\
\hline $6 \div-1$ & $1 . \vdots$ & 0141 & $i$ & & & & & $!$ & $\zeta \cos !$ \\
\hline$r-7$ & 3 & cian? & $\because$ & & & & & & min $: x: y$ \\
\hline$\therefore$ & ? & 96 & 3 & & & & & & $1112 \cdot$ \\
\hline$\because \quad !$ & 1.4 & 1600 & 82 & & & & & & S6010-Pan \\
\hline$\because \because$ & $x$ & 1936 & 82 & & & & & & (w) \\
\hline$G-r_{i}$ & $?$ & 1205 & 84 & & & & & & aif \\
\hline $3 \cdot \because \cdot \cdots$ & $\because$ & $\left(10^{\circ}\right)$ & 85 & & & & & & $\because-t: 1$ \\
\hline & & $17 i$ & 86 & & & & & & \\
\hline
\end{tabular}


CLIENT: WSRC

LOCATION: SRS-Land fill 740-6

PROJECT(1): Lanof al Geduro Sheet Taskd

PAGE: 3 OF:

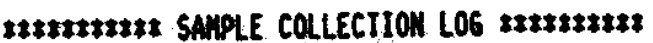

SAMPLER MAIE(S): DUM, AGC+RJE

\begin{tabular}{|c|c|c|c|c|c|c|c|c|c|c|}
\hline \multirow{3}{*}{$\begin{array}{l}\text { SAMPLE IDI } \\
\\
\text { GS.4 }\end{array}$} & \multirow{3}{*}{$\begin{array}{l}\text { DATE } \\
\text { :=2=2:2= } \\
1-8-91\end{array}$} & \multirow{3}{*}{$\begin{array}{l}\text { TINEE } \\
0620 \\
062 x=2\end{array}$} & \multirow{3}{*}{$\begin{array}{l}\text { SEO.1 } \\
=2=x=2 x== \\
87\end{array}$} & \multirow{3}{*}{$\begin{array}{l}\text { SAMPLE } \\
\text { DEPTH } \\
=:=\geq 2=:= \\
-0 .\end{array}$} & \multicolumn{3}{|c|}{ SAMPLE IYPE } & \multirow{3}{*}{$\begin{array}{c}\text { SAMPLE } \\
\text { SIZE } \\
2=2== \pm==2 \\
22 \mathrm{~mL}\end{array}$} & COHERTS & \multirow{3}{*}{$=\frac{C o c}{20}=$} \\
\hline & & & & & 6 & $=$ & $W_{= \pm= \pm}^{W}$ & & 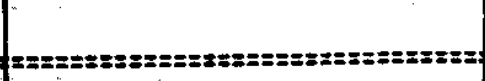 & \\
\hline & & & & & $\checkmark$ & & & & & \\
\hline$G S-1$ & $1-8$ & 1005 & 88 & i & $i$ & & & , & & $\mid-9-9$ \\
\hline$G S-2$ & $1-8$ & 1010 & 89 & $\vdots$ & & & & $j$ & - & $1:$ \\
\hline 65 & 1.8 & 1015 & 90 & & & & & $\because$ & & $i$ \\
\hline & $1-8$ & 1020 & 91 & & & & & 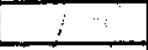 & & \\
\hline$G$ & 1.8 & 1405 & 92 & & & & & $i$ & & \\
\hline$r_{5}=$ & 1.8 & 1410 & 93 & & & & & & & \\
\hline & 1.8 & 1414 & 94 & $\vdots$ & & & & $\therefore$ & & \\
\hline .2 .4 & $1-8$ & 1417 & 95 & 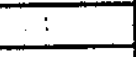 & & & & & $\therefore$ & \\
\hline$G S-4 A$ & $1-8$ & 1419 & 46 & & & & & & - & \\
\hline$G S-B L K^{3} q$ & $1-8$ & 1421 & 97 & $\therefore$ & & & & : & & 1 \\
\hline 65.1 & $1-x$ & 1750 & 98 & & & & & $!$ & Slight DR,zzle & \\
\hline $6=2$ & $1-8$ & 1755 & 99 & & & & & & & 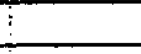 \\
\hline 6 & $1-8$ & 1800 & 100 & & & & & 1 & & \\
\hline $6 ?$ & $1-8$ & 17.05 & 131 & & & & & 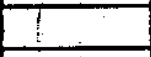 & & $!$ \\
\hline$?$ & $1-8$ & $=1: 50$ & 102 & & & & & & Cool, Sight Brecke DJM & $i$ \\
\hline 2 & $1-8$ & $21: 55$ & 103 & & & & & & $" 1$ & \\
\hline 6 & $1-8$ & 20.00 & 104 & & & & & $i$ & .". & $i$ \\
\hline 65 & $1-8$ & $22: 05$ & 105 & & & & & & $\therefore$ & 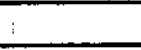 \\
\hline 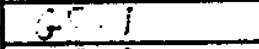 & $1-9$ & 0150 & 106 & & & & & & Fol & \\
\hline & $t-9$ & 10154 & 107 & & & & & &.$\cdot$ & \\
\hline $6:-2 A$ & $1-9$ & 0157 & 125 & & & & & & 4 & $i$ \\
\hline $6-\left(51 x^{5} 10\right.$ & $1-1$ & 2159 & $109=$ & & & & & & "1 & \\
\hline TS.? & $1-7$ & 0203 & 110 & & & & & &. & $\therefore$ \\
\hline 5.4 & $1-4$ & 2209 & III & & & & & & 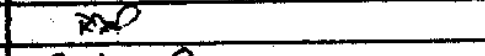 & $\because$ \\
\hline $6 \div-1$ & $1-9$ & 0605 & 12 & & & & & & Colo-Braezy & $i$ \\
\hline 2 & $1-q$ & $06 / 2$ & 113 & & & & & & $\cdots$ & 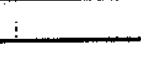 \\
\hline $65-3$ & $\sqrt{-9}$ & $06 / 3$ & 114 & & & & & & $\therefore$ & \\
\hline $65-4$ & $\sqrt{-G}$ & 0612 & 115 & & & & & & 11 & $A G G$ \\
\hline 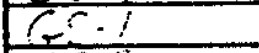 & $1-9$ & .0955 & 116 & & & & & & $\therefore$ & $i /-9-111$ \\
\hline 6.2 & $1-9$ & 11001 & i1? & & & & & & $\because$ & $C 21$ \\
\hline G; $:$ & $8-9$ & 1005 & $1 / 8$ & & & & & & $i$ & \\
\hline$G_{r} \cdot 2$ & $\sqrt{1-9}$ & 1010 & 119 & & & & & & $\because$ & 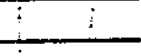 \\
\hline 65.16 & $1-y$ & 1012 & 120 & & & & & & $i$ & 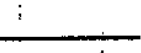 \\
\hline $6 \cdot \hat{E} \cdot:=$ & $-i$ & 1015 & 121 & & & & & & : & \\
\hline$G S-1$ & $1-9$ & 1,755 & 122 & & & & & & Ceol & \\
\hline$G \div .2$ & $1-8$ & 1400 & 123 & & & & & & & \\
\hline $65 \%$ & $1-9$ & 1405 & 124 & & & & & & & \\
\hline$f ? \cdot i$ & $1-3$ & 1410 & 125 & & & & & & & \\
\hline$\therefore$ & $11-7$ & 1755 & 126 & & & & & & & \\
\hline$\because$ & $\because ?$ & $: 1 \times 3,1$ & 427 & & & & & & & \\
\hline . & $1-i ;$ & To? & 128 & & & & & & & \\
\hline$\because$ & -4 & $72 \pi$ & 729 & & & & & & & \\
\hline
\end{tabular}


MICROSEEPS LTO.

CLIENT: WSRC

LOCATION: SRS-LANO fill 740-G

PROJECT(1): Geano Sheet TaSY II

PAGE: 4 OF:

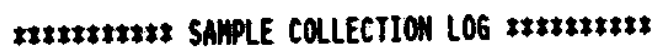

SAMPLER NAME(S): DJM+AGC +R)P

\begin{tabular}{|c|c|c|c|c|c|c|c|c|c|c|}
\hline SAMPLE IDI & DATE & IIINE & SEO.1 & SAMPLE & & LE & YYPE & SAMPLE & COMMENTS & \\
\hline & & $z=2=28=$ & & $\begin{array}{r}\text { DEPTH } \\
\text { =:=:s=:ss }\end{array}$ & $\begin{array}{c}6 \\
=2=z=2\end{array}$ & 5 & 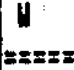 & $\begin{array}{c}\text { SIZE } \\
:=x= \pm=2=2=\end{array}$ & 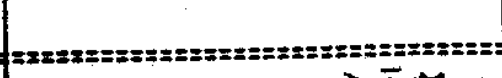 & \\
\hline GS. & $1-9$ & 2150 & 130 & $-0-$ & $\checkmark$ & & & $22 \mathrm{~mL}$ & DU.M & coc. \\
\hline $65-2$ & & 3155 & 131 & 7 & $=$ & & & $y$ & & \\
\hline$G i-2 A$ & 1 & 2156 & 132 & 1 & 1 & & & & & 7 \\
\hline$\frac{65-81 K^{3} 12}{G 5-3}$ & $\vdots$ & 2157 & 133 & I & $i$ & & & 7 & & 1 \\
\hline$\frac{65-3}{5-4}$ & 1 & $22: 05$ & 134 & 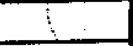 & $!$ & & & $I$ & & \\
\hline$\frac{78}{68}$ & $1-9$ & $22: 10$ & 135 & 1 & & & & 1 & DAM & 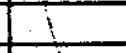 \\
\hline$\frac{6 S \cdot 1}{96}$ & $1-10$ & 0142 & 136 & L & 1 & & & 1 & $R \perp P$ & 5 \\
\hline 65.2 & 1.10 & 0146 & 137 & 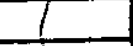 & 1 & & & $i$ & & $i$ \\
\hline $6=2$ & $\cdots 3$ & 0150 & 135 & 1 & & & & & 6 & \\
\hline$\frac{6-4}{2-4}$ & $1-10$ & 0154 & 139 & $!$ & & & & & $\mathrm{ESP}$ & \\
\hline $6 r \cdot 1$ & $1-10$ & 0600 & 140 & 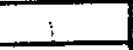 & & & & & Light $R_{A 1 n}-C_{0 L s}-L_{1 N 0}$ & $d y$ \\
\hline 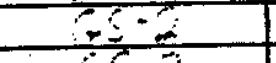 & $1-10$ & 0.604 & 141 & 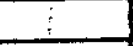 & & & & & & 1 \\
\hline $68-3$ & $(\cdot 1)$ & 0608 & 142 & ; & & & & & & \\
\hline $6 r-4$ & $1-12$ & 9613 & 143 & $\therefore$ & & & & & & \\
\hline $6 \cdot i$ & 1.io & $2 \times 15$ & 144 & & & & & & & $A G C$ \\
\hline$-1,+1$ & $1-10$ & $66: 8$ & 145 & & & & & & & \\
\hline 75 & $1-12$ & 1005 & 141 & & & & & & Cod wina, & \\
\hline $6 r .2$ & -2 & 1008 & 142 & & & & & & & \\
\hline $65-3$ & $\cdots 2$ & 1011 & 148 & & & & & & & \\
\hline 65.4 & $\therefore 3$ & 1015 & 149 & & & & & & & is \\
\hline$\because \because 1$ & 21 & 1355 & 150 & & & & & & & i \\
\hline$\frac{5}{2}=3$ & $\cdots 3$ & 14ee & 157 & & & & & & WARMELINAY & 1 \\
\hline $65 \cdot 3$ & $\cdots .1$ & 1403 & 152 & & & & & & & $\vdots$ \\
\hline $6-5-4$ & -1 & $1 \% 07$ & 153 & & & & & & & $i$ \\
\hline $65-1$ & -2 & 1255 & 154 & & & & & & $00,22 / c$ & $!$ \\
\hline $65-2$ & -3 & 1200 & 155 & & & & & & & $\vdots$ \\
\hline $65-2 A$ & -1 & 1802 & 156 & & & & & & & $i$ \\
\hline $65-8 L x^{4}+4$ & $\therefore$ & 1804 & 157 & & & & & & & $i$ \\
\hline 65.3 & $1, \ldots$ & 1808 & 158 & & & & & & & 1 \\
\hline$G S-4$ & $(-10$ & 1811 & 159 & & & & & & & $t$ \\
\hline $6 !-1$ & $i-12$ & $10: 15$ & 160 & & & & & & Laght Ra, & \\
\hline $6: 2$ & $\because-i)$ & $10: 20$ & 161 & & & & & & " & \\
\hline 65.3 & $i-2$ & 12.25 & 162 & & & & & & $\because$ & \\
\hline $65-4$ & $(-1)$ & $10: 30$ & 163 & & & & & & $\because$. & \\
\hline 6.5 & $\therefore$ & 10102 & 164 & & & & & & 50 & \\
\hline 65.2 & & 0143 & $165^{\circ}$ & & & & & & & \\
\hline $5 \div ?$ & & 010 & 160 & & & & & & Lf RAij- & \\
\hline$\therefore \quad \cdot$ & & 10112 & $\because:$ & & & & & & $70-$ & \\
\hline$G:: 1 \mathrm{~L}$ & & 115 & $i \quad \therefore$ & & & & & & 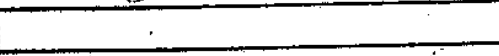 & \\
\hline$\because-81.5$ & & $3.1 \%$ & $\ldots i$ & & & & & & 6 & \\
\hline$\therefore \quad 1$ & & $96 \%$ & 92 & & & & & & $P n$ & \\
\hline$\therefore \quad$. & & $\therefore \ldots$ & -1 & & & & & & & \\
\hline$\ldots$ & & $\because \because$ & $i \dot{i}$ & & & & & & & $7 \div$ \\
\hline
\end{tabular}


SAMPER MUNE(S): $D J M+A G C$

\begin{tabular}{|c|c|c|c|c|c|c|c|c|c|c|}
\hline SAMPLE IDI & DAtE & IIIKE & $\sec .1$ & $\operatorname{sipe}$ & & QE & $\operatorname{mex}$ & SMPLE & Coments & \\
\hline & $2: 2=2 x=$ & z2x2:z=3: & & 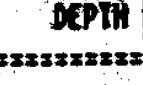 & $\begin{array}{l}6 \\
=2 x z z z\end{array}$ & 5 & $y$ & SIZE & 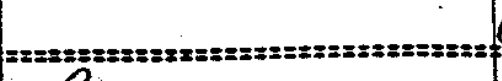 & $\operatorname{coc}=$ \\
\hline GS-4 & $|-1|$ & $0 \times 0$ & 123 & -0 & $\checkmark$ & & & $22 \mathrm{~mL}$ & PAN & \\
\hline $65-1$ & $\because$ & 1001 & 174 & 1 & 1 & & & 1 & 1. & \\
\hline $6-5.2$ & $i$ & 1004 & 175 & ( & i & & & 1 & $\therefore$ & \\
\hline $6-5-3$ & & 1010 & 176 & 1 & $i$ & & & $i$ & 1. & \\
\hline $65-4$ & & 1015 & 177 & & 1 & & & $i$ & 11 & \\
\hline $65-1$ & & 1402 & 178 & 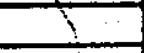 & 1 & & & $i$ & Heavy Ram. Streomawno & \\
\hline $65 \cdot 2$ & & 1406 & 179 & 1 & $\because$ & & & 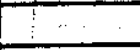 & 1 & \\
\hline $6=-2 A$ & & 1408 & $180-$ & I & $\therefore$ & & & . & & \\
\hline$C 5 \cdot B L \times 16$ & & 1410 & 181 & $!$ & 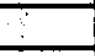 & & & & $\therefore$ & \\
\hline $6-3$ & & 1415 & 182 & $\vdots$ & $!$ & & & : & & \\
\hline $65-4$ & & 1418 & 183 & & & & & 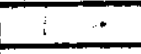 & & \\
\hline $65-1$ & & 1820 & .84 & & & & & & $2 \bar{M} A G C$ & \\
\hline $65 \cdot 2$ & & 2525 & 185 & $!$ & & & & & 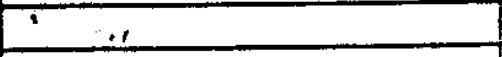 & \\
\hline $65-3$ & & 1530 & 186 & 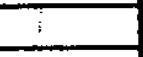 & & & & & 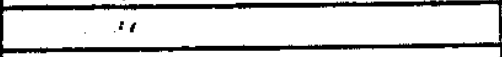 & \\
\hline $65-4$ & & 1835 & .87 & & & & & & $n$ & \\
\hline$G S .1$ & & $22: 10$ & 188 & & $\vdots$ & & & & $\overline{D T M}$ & \\
\hline$G S-2$ & & $22: 15$ & 189 & & & & & & 1. & \pm \\
\hline$G s-3$ & & 2220 & 190 & & & & & & 11 & \\
\hline $65-4$ & & $22: 25$ & 191 & & & & & & $\cdots$ & \\
\hline$C_{Y}-4 A$ & & $72: 27$ & 192 & & & & & & 11 & \\
\hline$\left(2\left(-\beta L k^{*} \mid\right)\right.$ & & $32: 29$ & 193 & & & & & & 11 & \\
\hline $65 \cdot 1$ & $1+2$ & $0^{\prime}: 35$ & 194 & & $\vdots$ & & & & 1. & $i$ \\
\hline $6 r-2$ & & 10140 & .95 & & & & & & $\because$ & \\
\hline GS-3 & & 0145 & 136 & & & & & & 11 & \\
\hline 65.4 & & $015^{\circ}$ & 149 & & & & & & 11 & \\
\hline $6: 1$ & & 0603 & 198 & & & & & & $A G C \cdot L_{1} g h+R_{a} \cdot n \cdot C o l m$ & \\
\hline$G \vdots \cdot \ddot{z}$ & & 0606 & 199 & & & & & & & \\
\hline $5 \div 3$ & & 0610 & 200 & & & & & : & & 606 \\
\hline$\therefore y$ & & 0619 & 201 & & & & & & & \\
\hline $6:-1$ & & 0958 & 202 & & & & & & AGC-:Nwacy - WAre - & $i$ \\
\hline $6 r-2$ & & 1001 & 203 & & & & & & 11 & $\dot{i}$ \\
\hline $6 S-2 A$ & & 1003 & 204 & & & & & & & $\bar{\vdots}$ \\
\hline $65-62 \times 8$ & & 1005 & 205 & & & & & & 1 & $i$ \\
\hline $65-3$ & & 1009 & 206 & & & & & & & $i$ \\
\hline 65.4 & & 1012 & 207 & & & & & & & \\
\hline$G \cdot-1$ & & 135 & 6.7 & & & & & & $i$ & \\
\hline l, $=-\dot{\alpha}$ & & 1401 & 209 & & & & & & & \\
\hline $3 \cdot 2$ & & 925 & $2 \cdot i$ & & & & & & & \\
\hline$\because 6$ & & 199 & $21:$ & & & & & & & \\
\hline$r$ & & 1755 & 212 & & & & & & & \\
\hline 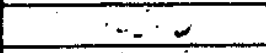 & & 1258 & 213 & & & & & & & \\
\hline$\because \div$ & & $\angle x \cap 1$ & 314 & & & & & & . & \\
\hline$\therefore$ & & 1496 & 215 & & & & & & & \\
\hline
\end{tabular}


MICANSEEPS LID.

aien: WSRC

Locartow: SRS-LANofill $740-6$

ProJECI(1): Grouwosheet - Insil ${ }^{3} \mid$

PMeE: -6 of:

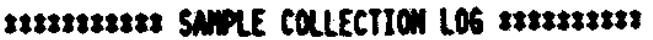

SAMPLER MAME(S): D) $m+A G C$

\begin{tabular}{|c|c|c|c|c|c|c|c|c|c|c|}
\hline SAMPLE IDI & OAIE & TIME & set:1 & SAivite & & LE & IPPE & SAMPE & COMENTS & \\
\hline & & & & 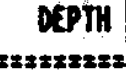 & .6 & 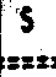 & $y_{=2 x=x}^{1}$ & 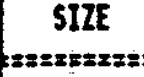 & 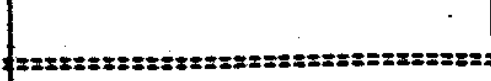 & 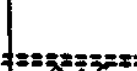 \\
\hline$G S-4 A$ & $1-12$ & 1809 & $2 / 6$ & Oft. & $\checkmark$ & & & $22 \mathrm{~mL}$ & Winoy twakm & $26 \mathrm{C}$ \\
\hline$C 5 \cdot B L K^{2} 19$ & $1-12$ & 18,3 & 217 & & 1 & & & & 111. & $\div 1$ \\
\hline GS & $1-12$ & 2200 & $2 / 8$ & $i$ & & & & & & 1 \\
\hline GS & 1.12 & 2205 & 2,9 & & $t$ & & & $i$ & & $T$ \\
\hline 65 & $1-12$ & 2210 & 200 & & I & & & & & 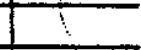 \\
\hline $65-4$ & $1-12$ & 22,4 & 221 & $\therefore$ & 1 & & & $\because$ & & \\
\hline$G S-1$ & $1-13$ & 0125 & 222 & & $T$ & & & $i$ & & $i$ \\
\hline G5.2 & $1-13$ & 0129 & 223 & & 1 & & & & & \\
\hline GS-3 & 1,13 & 0133 & 224 & & 1 & & & & 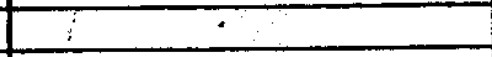 & \\
\hline GS.4 & $1-13$ & 0137 & 205 & & 1 & & & & & \\
\hline $\mathrm{CS}-1$ & $1-13$ & 0608 & 226 & & & & & & Colo + Calm & \\
\hline $65-2$ & $1-13$ & 0611 & 227 & & $i$ & & & & 11 & \\
\hline $65.2 A$ & $1-13$ & 0613 & 224 & & 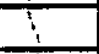 & & & & ... & $i$ \\
\hline $6 S-B L 20$ & $1-13$ & 0615 & 229 & $\therefore$ & $I$ & & & & 1. & $1:$ \\
\hline $65-3$ & $1-13$ & 0618 & 230 & & $T$ & & & & 11 & $1-13-9_{i}$ \\
\hline $65-4$ & $1-13$ & 0621 & 231 & & $\therefore$ & & & & 1, & $A G C$ \\
\hline 65.1 & $1-13$ & 1004 & 232 & & 1 & & & & Sury - Cool-Seeczy & 2 \\
\hline 65.2 & $1-13$ & 1007 & 233 & & 1 & & & & . & 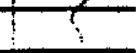 \\
\hline $6 ? \cdot 3$ & $1-13$ & 1011 & 234 & & T & & & & $\because$ & $\bar{t}$ \\
\hline $6 r \cdot 4$ & $\sqrt{-13}$ & $10 / 6$ & 235 & & 1 & & & & 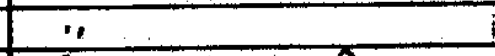 & 1 \\
\hline$G S-1$ & $1-13$ & 1407 & 236 & & $!$ & & & & $S_{V N R Y}-w_{A K M} \cdot P_{R E C Z Y}$ & 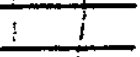 \\
\hline $6-5-2$ & $1-13$ & 1410 & 237 & & $T$ & & & & 17 & $T$ \\
\hline $65-3$ & $1-13$ & $14 / 4$ & 238 & & ? & & & & .1 & 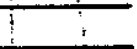 \\
\hline $65 \cdot 4$ & $1-13$ & $1 / 418$ & 234 & & $i$ & & & & 11 & $i$ \\
\hline$C S-4 A$ & $7-13$ & 1420 & 240 & & 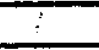 & & & & 11 & $i$ \\
\hline$G S-B\left(x^{2}\right)$ & $1-13$ & 1422 & 24 & & $i$ & & & & 11 & $i$ \\
\hline $65-1$ & 173 & 1753 & 242 & & & & & & cool-calm & \\
\hline $6.5 \cdot 2$ & $1 / 3$ & 1757 & 243 & & 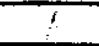 & & & & & $i$ \\
\hline $65-3$ & $1-13$ & 1801 & $24 y$ & & $\vdots$ & & & & & $i$ \\
\hline$(x)-4$ & $7-13$ & 1805 & 245 & & 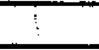 & & & & & \\
\hline$G=1$ & $1-13$ & 1040 & 241 & & & & & & & $!$ \\
\hline $6+2$ & $1-13$ & 1050 & 24) & & $\because$ & & & & & $!$ \\
\hline G5.? & $1-13$ & 1103 & 248 & & $\bar{i}$ & & & & & \\
\hline 65.4 & $1+3$ & $11: 07$ & 249 & & & & & & & \\
\hline $6-1$ & 174 & 0,47 & 750 & & & & & & & \\
\hline $6 i d$ & $1-14$ & $015^{2}$ & 251 & & & & & & & \\
\hline $6-2 i$ & $1-14$ & 0154 & 252 & & & & & & & \\
\hline $6-3 \cdot \cdot \cdot \cdot=$ & $1 \cdots$ & 020 & 20,3 & & & & & & & \\
\hline$\therefore .1$ & $: \div$ & 0705 & 259 & & & & & & & \\
\hline $6: \cdots$ & 1 & 0710 & 255 & & & & & & & \\
\hline $5=-1$ & $1-1-1$ & $06 / 12$ & 256 & & & & & & ColD-Chim - Frex, tom & Sńc.:1. \\
\hline $3: 2$ & 1.14 & 0615 & 257 & & & & & & & $i$ \\
\hline $75 \div$ & $1-14$ & $\alpha, 1 x$ & 258 & & & & & & & $1: 8$ \\
\hline
\end{tabular}




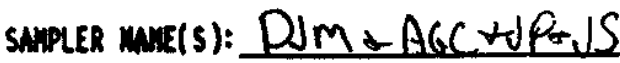

\begin{tabular}{|c|c|c|c|c|c|c|c|c|c|c|}
\hline SAHPLE IDI & MATE & III & SEO.1 & SMMPLE & & LE I & & SAMPLE & COMNENTS & \\
\hline :s:2: & & & & 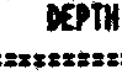 & 6 & $S$ & $y$ & SIIE & 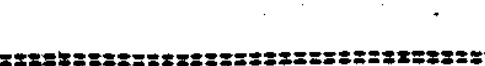 & $=0$ \\
\hline C.S. 4 & L- & 0,0622 & 259 & $-0-$ & $\checkmark$ & & & $22 \mathrm{~mL}$ & Colo-Calm - Frost or Sheet & $\operatorname{coc}$ \\
\hline$G S-1$ & $E$ & 1008 & 260 & & & & & & Siney $\left.C_{A L M}-C_{0} O\right)$ & \\
\hline css-2 & $1-14$ & 1011 & 26.1 & 1 & & & & & $r$ & \\
\hline GS & $1-14$ & 1013 & 262 & & & & & & .1 & \\
\hline$\frac{-4}{-4 A}$ & $\sqrt{-14}$ & 1017 & 263 & & & & & & it & \\
\hline$-4 A$ & $1-1 y$ & 1019 & 264 & I & & & & & 7 & \\
\hline$\frac{G S-\beta L x^{*} 23}{65-1}$ & $1+4$ & 1021 & 265 & & & & & & 11 & \\
\hline $65-1$ & $1-14$ & 1410 & 266 & & & & & & $25 M \quad s-7$ & 1 \\
\hline$\frac{G 5-2}{6-5-3}$ & $1-14$ & 14,5 & 267 & & & & & & & \\
\hline$\frac{65-3}{C 5-4}$ & $1=14$ & 1400 & 268 & 1 & & & & & & \\
\hline$\frac{5-4}{-1}$ & $1-14$ & 1425 & 264 & & & & & $\cdot$ & & \\
\hline$\frac{65-1}{65-2}$ & $1-14$ & 1800 & 270 & & & & & & AGC - WAPM - $\mathrm{CAlm}$ & 1 \\
\hline$\frac{65-2}{6}$ & $1-14$ & 1804 & $2 \geqslant 1$ & & & & & & $\therefore$ & \\
\hline$\frac{-3}{-4}$ & $1-14$ & 1809 & 272 & & & & & & $\because$ & \\
\hline$\frac{-4}{5-1}$ & $1-14$ & +14 & 273 & & & & & & $\because$ & \\
\hline$\frac{G}{G}$ & $\frac{1-14}{1-14}$ & $\frac{2700}{2205}$ & $\frac{274}{275}$ & & & & & & $\because$ & \\
\hline $2 A$ & $1-14$ & 2207 & 276 & & & & & & & $\frac{1}{i}$ \\
\hline$-84 k=24$ & $1-14$ & 2209 & 277 & & & & & & & \\
\hline 3 & $1-14$ & 2214 & 278 & & & & & & & 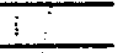 \\
\hline $6.5-4$ & $1-14$ & 247 & 279 & & & & & & & \\
\hline C.5-1 & $1-15$ & 0155 & 28 & & & & & & & :1 \\
\hline $65 \cdot 2$ & $1-15$ & 0159 & $281-$ & & & & & & & \\
\hline 65.3 & $1-15$ & 0205 & 282 & & & & & & & 1 \\
\hline $6 \div-4$ & -15 & 0210 & 283 & & & & & & & i: \\
\hline $6 s-1$ & 175 & $0615^{-}$ & $28 \%$ & & & & & & Cool - CAlm & $!$ \\
\hline Gs.a & 1.15 & 0618 & 285 & & & & & & & \\
\hline $65-3$ & 15 & 0621 & 286 & & & & & & $\therefore$ & \\
\hline$G^{5-4}$ & $1-15$ & 0624 & 282 & & & & & & $\because$ & \\
\hline GS $-4 A$ & $1-15$ & 0626 & 288 & & & & & & $\cdots$ & $i \operatorname{CoC}$ \\
\hline G5-81k25 & $1-15$ & 0629 & 289 & & & & & & 1. & IACG -1 \\
\hline $6:-1$ & $1-15$ & 1004 & 290 & & & & & & 20 & - \\
\hline $6 r-2$ & $1-15$ & 1007 & 291 & & & & & & & \\
\hline $6 \div-3$ & $1-15$ & 1010 & 292 & & & & & & & 1 \\
\hline $6 s-4$ & 1.15 & 1014 & 293 & & & & & & & \\
\hline$\therefore-1$ & $1+2$ & 1407 & 294 & & & & & & & 1 \\
\hline$G \because=$ & $-1-$ & 1411 & 295 & & & & & & & \\
\hline & $\cdots$ & 1414 & 296 & & & & & & & \\
\hline 6.1 & $\therefore$ & 1417 & 297 & & & & & & & \\
\hline $6 \cdots$ & & 1801 & 298 & & & & & & $\therefore$ & \\
\hline 6. & & 1804 & 299 & & & & & & & \\
\hline$S=1$ & & 1506 & $\because 00$ & & & & & & & \\
\hline 50.6 & & $3 x$ & 301 & & & & & & & \\
\hline
\end{tabular}


Pise: $\overline{8}$ of:

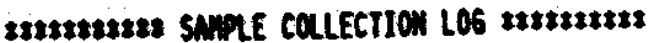

SAMPLER Mare(s): $A G C+D J M+R J F$

\begin{tabular}{|c|c|c|c|c|c|c|c|c|c|}
\hline SAMPLE IOI & DATE & TIME & 560.8 & SAMPE & & LEI & & SMPPLE & COMWENTS \\
\hline & & & & $\underset{\text { DEPPTH }}{\text { DEsz:sz }}$ & $\frac{6}{6 x z=z}$ & $\mathbf{s}$ & 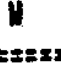 & 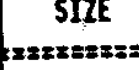 & 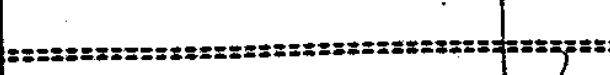 \\
\hline $\mathrm{Cl}_{i}-$ & $1-15$ & 1812 & 302 & $-0-$ & 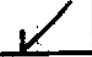 & & & $22 \mathrm{~mL}$ & $2++r-\ln l m$ \\
\hline$\frac{65}{65}$ & $1-15$ & 1815 & 303 & & & & & & \\
\hline$\frac{65-1}{65-2}$ & 1.15 & 2205 & 304 & & $!$ & & & & RAIN!!! \\
\hline$\frac{65-2}{6-5-3}$ & $1-15$ & 2210 & 305 & ' & 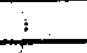 & & & & 11 \\
\hline$\frac{\cos 3}{65-4}$ & $1-15$ & 2215 & 306 & & 1 & & & & II SUCKed WATCR (MO SAmple) \\
\hline$\frac{6-7}{65-1}$ & $\frac{7}{1-16}$ & 0144 & 307 & & & $E$ & 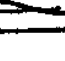 & $=$ & \\
\hline$\frac{65-2}{65.3}$ & $1-16$ & 0,149 & 308 & & & & & & \\
\hline$\frac{65-3}{6-5-4}$ & $1-16$ & $0 ! \leq 4$ & 309 & & $\vdots$ & & & & \\
\hline$\frac{C-5-4}{C 5-4-A}$ & $1-16$ & $=$ & & $=$ & $E$ & $\equiv$ & $=$ & $=$ & 3 whter ino \\
\hline$\frac{C 5-4-A}{65-B 1 k^{5} 27}$ & $1-16$ & & & & & & & & 1 sericie. \\
\hline$\frac{65-131 k^{n} 27}{65-1}$ & $\frac{1-16}{1-16}$ & Bion & $3 / 0$ & & & & & & 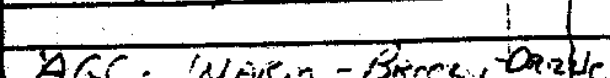 \\
\hline$\frac{65-1}{6.5 \cdot 2}$ & $\frac{1-16}{1-16}$ & & $\frac{311}{312}$ & & & & & & 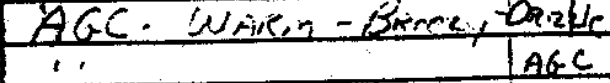 \\
\hline$\frac{52}{15-3}$ & $\frac{1-16}{1-16}$ & $\frac{0603}{0611}$ & $\frac{5 / 2}{3 / 3}$ & & & - & & & $\frac{A G C}{C S C}$ \\
\hline $65-4$ & $i-16$ & & & & & & & & WATER - Na SAmp/C \\
\hline$\frac{6 i-1}{6-2}$ & $1-16$ & 1002 & $3 / 4$ & & & & & & WARM - WeT NoRAn \\
\hline 6.2 & $1-16$ & 1006 & 315 & & & & & & \\
\hline $6-3$ & $1-16$ & 1010 & 316 & & $:$ & & & & \\
\hline$\frac{6-40}{65-1}$ & $1-16$ & & & & & & & & WATER WLine No Samp'e \\
\hline$\frac{65-1}{(-5-2}$ & $1-16$ & 1402 & 317 & & & & & & Suawy $x$ Wuinoy + warm \\
\hline$\frac{65-2}{6:-2 A}$ & $1-16$ & 1406 & 318 & & & & & & , \\
\hline$\frac{G ;-2 A}{G S-E !<28}$ & $1-16$ & 1408 & $319=$ & & & & & & \\
\hline$\frac{G 5-12 \times 28}{65-3}$ & $\frac{1-16}{1-16}$ & 2410 & 320 & & & & & & - \\
\hline$\frac{65-3}{65-4}$ & $\frac{1-6}{1-16}$ & $\frac{1414}{1.140}$ & $\frac{321}{322}$ & & & & & & Oratreench Deameowatere \\
\hline $65-i$ & $\frac{1-12}{1-12}$ & $\frac{1750}{1750}$ & $\frac{322}{323}$ & & & & & & 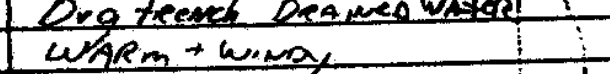 \\
\hline $6: 2$ & $1-16$ & 1755 & 324 & & & & & ! & conim \\
\hline $6 ;-3$ & $1-16$ & 1800 & 325 & & & & & & \\
\hline 65.4 & $1-1$, & 1804 & 326 & & & & & & \\
\hline$\therefore i$ & $\therefore$ & 2155 & 327 & & & & & & 1 \\
\hline $65-2$ & $\cdots$ & 2200 & 328 & & & & & & \\
\hline $65-3$ & $\theta$, & 2203 & 329 & & & & & & \\
\hline 65.4 & $\because$ & 2206 & 330 & & & & & & \\
\hline $6 ? .4 i$ & 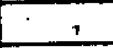 & 22008 & 331 & & & & & & \\
\hline $6 \% \cdot 1$ & $1 ;$ & $22 \% 0$ & $\frac{332}{333}$ & & & & & & DSM \\
\hline $6 \div-1$ & $1-1$ & 0230 & $\frac{333}{394}$ & & & & & & \\
\hline 6 & & $\frac{0234}{0=32}$ & $\frac{234}{33}$ & & & & & & \\
\hline$\therefore$ & & $1>42$ & $\therefore$ & & & & & & $\therefore$ \\
\hline$\ldots 1$ & & 0607 & 337 & & & & & & 116 \\
\hline & & 0609 & 328 & & & & & & \\
\hline & & .613 & 530 & & & & & & \\
\hline
\end{tabular}


pief: 9 of:

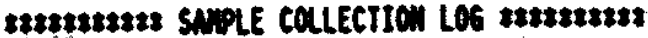

SAMPLER MUE(S): $A G C+D J M+R J P N S$

\begin{tabular}{|c|c|c|c|c|c|c|c|c|c|c|}
\hline SAMPLE IOS & DATE & IIME & 560.1 & Simine & & ZE & The & SMPLE & COMnENTS & \\
\hline & & & & 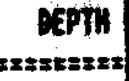 & 6 & 5 & 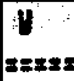 & 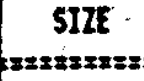 & 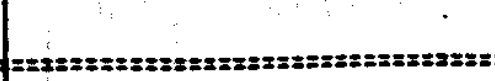 & AGG: \\
\hline CS. 4 & $1-12$ & 0618 & 340 & -0 & $\checkmark$ & & & $2 \operatorname{Ln}_{2}$ & & 6069 \\
\hline $6 S-1$ & $1-17$ & 1009 & 391 & 1 & 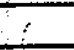 & & & & & \\
\hline$c s-2$ & $1-12$ & $10 / 2$ & 342 & 7 & & & & $\because$ & & 3 \\
\hline $\cos 2 A$ & $1-12$ & 1014 & 343 & & & & & & 1 & \\
\hline $55-B L x^{2} 30$ & $1-17$ & $10 / 6$ & 344 & & & & & & & \\
\hline $6 s \cdot 3$ & $1-17$ & 1018 & 345 & 1 & 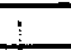 & & & & & \\
\hline $65-4$ & $1-17$ & 1021 & 346 & $i$ & $i$ & & & & & \\
\hline$G S-1$ & $1-17$ & 1410 & 347 & 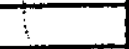 & & & & & Swinger waye sing bet & $i$ \\
\hline $65 \cdot 2$ & $1-17$ & $14 n 2$ & 348 & & & & & & $: \quad: \quad$ surear & $T$ \\
\hline $65-3$ & $1-17$ & 1426 & 349 & & & & & 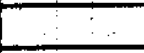 & 11 & \\
\hline $65-4$ & $1-17$ & 1430 & 350 & & 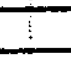 & & & $\dot{-}$ & 11 & \\
\hline$G S-1$ & $1-17$ & 1759 & 351 & & & & & 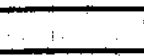 & $y A \alpha m-C_{A} / m \quad A G C$ & \\
\hline $6: .2$ & $1-12$ & 1802 & 352 & $\dot{3}$ & & & & & 1 & \\
\hline $65-3$ & $1-17$ & 1806 & 253 & & & & & & Y & $i$ \\
\hline $65 \cdot 4$ & $1-17$ & 1810 & 354 & & & & & & 11 & \\
\hline $6 j-4 A$ & $1-i\rangle$ & 1812 & 355 & & $\vdots$ & & & & $\because$ & \\
\hline $6 S-82 K+31$ & $|-1\rangle$ & 1813 & 356 & & & & & & $\ldots$ & \\
\hline GS-1 & $1-17$ & 2200 & 357 & & & & & & & 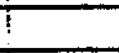 \\
\hline $65 \cdot 2$ & $1-17$ & 2203 & 358 & & & & & & & \\
\hline $65 . ?$ & $1-12$ & 2206 & 359 & & & & & & & \\
\hline $65-7$ & $1-12$ & 2209 & 360 & & & & & & & \\
\hline$G 5-1$ & $1-18$ & 0132 & 361 & & & & & & BJP & \\
\hline 65.2 & $1-18$ & 0141 & 362 & & & & & & $x$ & $\therefore$ \\
\hline$G S \cdot ?$ & $1-18$ & 0145 & 363 & & & & & & 21 & \\
\hline 65.7 & $1-18$ & 0,50 & 364 & & & & & & $x a$ & \\
\hline $6:-1$ & $1-18$ & $06 / 0$ & 365 & & & & & & $A 6 C$ & \\
\hline$G=2$ & $1-18$ & 0614 & 366 & & & & & & $\mathrm{CoO} \cdot \mathrm{CAlm}$ & i \\
\hline$G=2 A$ & $1-18$ & 0616 & 367 & & 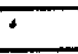 & & & & & \\
\hline$\left.x^{1}-6 x^{2}\right)^{2}$ & $1-18$ & 0618 & 368 & & & & & & & $1-184$ \\
\hline$\therefore 3$ & $1-18$ & 0621 & 369 & & & & & & & $\operatorname{cox}$ \\
\hline$C_{x}^{\prime}-4$ & -17 & 0624 & $3>0$ & & & & & & & $A G C$ \\
\hline $6-1$ & $1-18$ & 1004 & 371 & & & & & & Govor-indy-Cod & \\
\hline $6:-2$ & 1.18 & 1007 & 372 & & & & & & 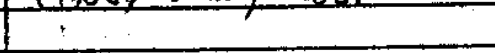 & \\
\hline$G \cdot ?$ & $1-18$ & 1011 & $3>3$ & & & & & & . & \\
\hline $5=4$ & $1-18$ & 1015 & 374 & & & & & & $\therefore \quad 2-2$ & \\
\hline $6+1$ & $1 \cdot 18$ & 1357 & 375 & & & & & & Sunay-Wincy - Navm & \\
\hline 5.2 & $1-18$ & 1400 & 376 & & & & & & 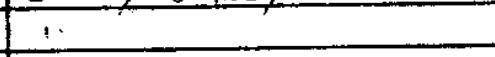 & \\
\hline$\div .3$ & 17 & 1403 & 322 & & & & & & - & \\
\hline 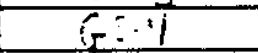 & $: 3$ & 1707 & 378 & & & & & & & \\
\hline $1 \hat{\epsilon}$ & $\because$ & 1411 & 376 & & & & & & $\because$ & \\
\hline $6-4=28$ & $i \quad i$ & 1412 & 380 & & & & & & 11 & \\
\hline $5 \div$ & $\because 3$ & 1807 & 381 & & & & & & & \\
\hline$G_{-}:-$ & $\because$ & $3 ! 5$ & $38:$ & & & & & & & \\
\hline
\end{tabular}


MICROSEEPS LID.

aIEm: $W S R C$

Locartow: SRS LAno G.11 740-6

PROJECT(1): Grouna sheet TASKM I

PAGE: 10 of:

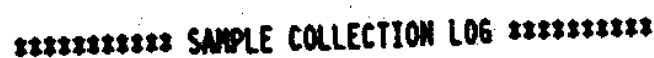

SAMPLER MMNE(S): $A G C+D J M+R J P$

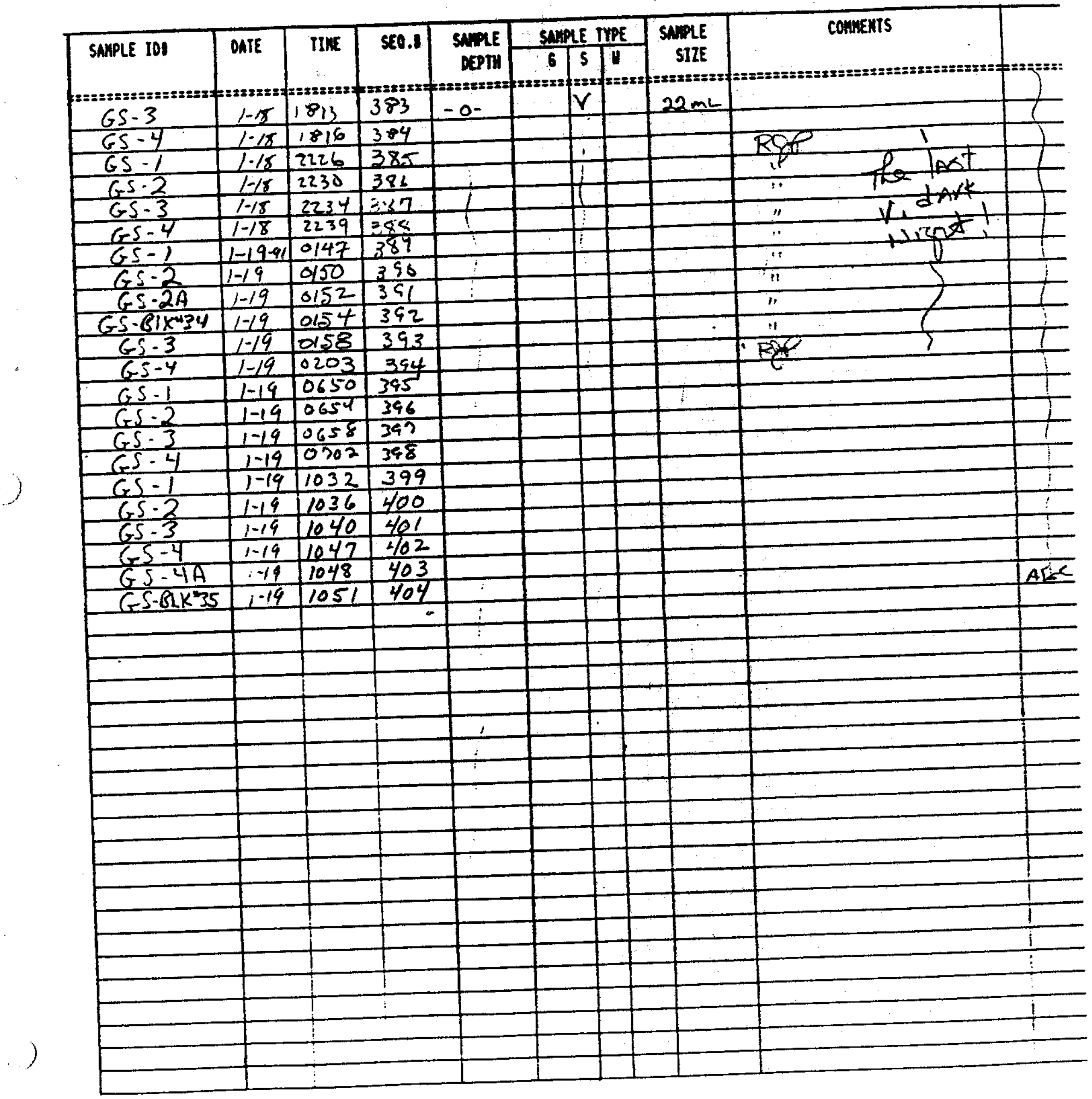

